\title{
Evaluating the use of High-Frequency Radar coastal currents to correct Satellite Altimetry \\ by
}

\section{Carolyn J. Roesler}

M.S., ENSPS Strasbourg France, 1991

M.S., University of Boulder Colorado, 2009

\author{
A thesis submitted to the \\ Faculty of the Graduate School of the \\ University of Colorado in partial fulfillment \\ of the requirements for the degree of \\ Doctor of Philosophy \\ Department of Aerospace Engineering


This thesis entitled:

Evaluating the use of High-Frequency Radar coastal currents to correct Satellite Altimetry written by Carolyn J. Roesler

has been approved for the Department of Aerospace Engineering

Dr. William J. Emery

Dr. Robert R. Leben

Date

The final copy of this thesis has been examined by the signatories, and we find that both the content and the form meet acceptable presentation standards of scholarly work in the above mentioned discipline. 
Roesler, Carolyn J. (Ph.D., Aerospace Engineering)

Evaluating the use of High-Frequency Radar coastal currents to correct Satellite Altimetry Thesis directed by Dr. William J. Emery

Conventional satellite altimetry provides very satisfactory results in open ocean conditions. Yet in coastal zones altimetry faces various problems, including less reliable standard corrections, and altimeter waveform (return echo) degradation arising from the rapid changes in the sea state or land contamination in the altimeter footprint. Different retracking (technique to retrieve geophysical information from the waveforms) methods have been developed, but the optimal method may turn out to be a combination of several retrackers, and may depend on the sea state. The coastal High-Frequency Radar (HFR) ocean surface currents are evaluated to test if they can be exploited to validate the coastal altimeter Sea Surface Height (SSH) measurements.

A method to retrieve the geostrophic velocities from the HFR sea surface currents is established in the offshore region where the altimeter data are trusted. At the large mesoscales this method provides HFR SSH that are in very good agreement with altimetry gridded products with correlations larger than 0.8. Along a Jason-1 or Jason-2 altimeter track the agreement depends essentially on the wind history, and a limitation is the smooth field produced by the method. Nevertheless, more than half the cases match with a mean rootmean-square difference of $2.5 \mathrm{~cm}(6.7 \mathrm{~cm} / \mathrm{s})$ in sea level anomaly (across track geostrophic velocity anomaly).

Even without land contamination in the altimeter footprint, the waveforms may be degraded by non-homogeneous ocean dynamics, and during rain or low wind events. The HFR surface currents provide information on the sea surface height, so that various altimetry retrackers are tested under those conditions. Also referencing to the HFR SSH, several coastal retrackers can be evaluated in the near-shore regions. 
Even though these studies demonstrate the value of HFR as a tool to correct coastal satellite altimetry measurements over a section of an altimeter track, which is an improvement compared to sparse in-situ measurements, caution must be taken when generalizing the methodology since the inferred geostrophic HFR datasets may still be contaminated by ageostrophic components, especially during high wind events. 


\section{Dedication}

To my parents. 


\section{Acknowledgements}

There are many people I would like to thank for helping me complete this work. I would like to thank all my committee members: Dr. Bill Emery, Dr. Robert Leben, Dr. John Wahr, Dr. Jeffrey Thayer and Dr. Jean Tournadre. My sincerest thanks go to my advisor, Bill Emery, who has always been patient, helpful and supportive. I am grateful to my committee member, Jean Tournadre, for giving me the opportunity to visit Ifremer and for his guidance during this work. I also would like to acknowledge the Coastal Altimetry group for their encouragement.

My thanks go to the friendly team members in CCAR for their help and advice, particularly to the people who worked with me in the lab, both past and present, Dan Baldwin, Ian Crocker, Waqas Qazi and Sandra Castro. 


\section{Contents}

\section{Chapter}

1 Introduction 1

1.1 Introduction . . . . . . . . . . . . . . . . . . . 1

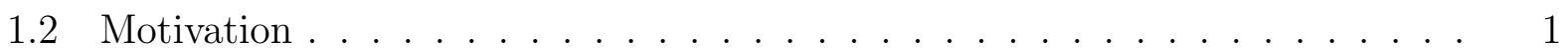

1.3 Coastal altimetry background . . . . . . . . . . . . . . . . . . 4

1.4 Objectives . . . . . . . . . . . . . . . . . . 7

1.5 Data sets . . . . . . . . . . . . . . . . . . . . 8

1.5.1 High-frequency coastal radars . . . . . . . . . . . . . . 8

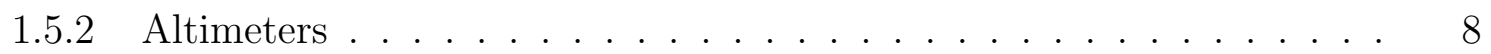

1.6 Dissertation Overview $\ldots \ldots \ldots \ldots$

2 Satellite Altimeter and HFR measurements and Data sets 11

2.1 Satellite Altimetry . . . . . . . . . . . . . . . . . . . 11

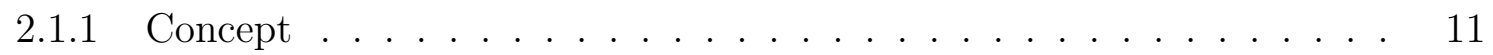

2.1.2 Waveform contamination .................. 13

2.1.3 Altimetric sea-level height corrections . . . . . . . . . . . . . 16

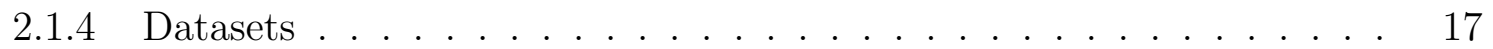

$2.2 \mathrm{HFR} \ldots \ldots \ldots \ldots \ldots \ldots$

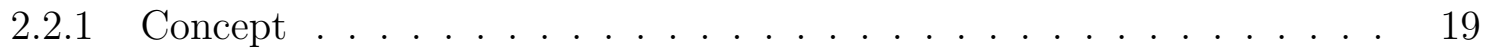

2.2.2 Sea Doppler Spectrum and sea surface current measurements . . . . . 20 
2.2.3 HFR Systems . . . . . . . . . . . . . . . . . . 22

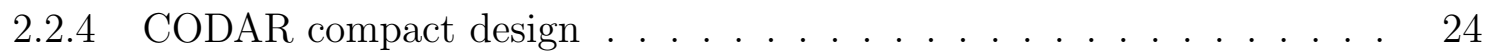

2.2.5 Maps of Sea surface vector currents . . . . . . . . . . . . . . 25

2.2.6 Depth extent of HFR surface currents . . . . . . . . . . . . . . 25

2.2.7 Stokes Drift or wave-induced currents . . . . . . . . . . . . . 26

2.2.8 Measurements errors . . . . . . . . . . . . . . . . . . 27

2.2.9 HFR dataset . . . . . . . . . . . . . . . . . . . 28

3 Dynamics in the California Current System 30

3.1 Introduction . . . . . . . . . . . . . . . . . . . . 30

3.2 California Current System . . . . . . . . . . . . . . . . . . 30

3.3 Spatial and temporal characteristics of the Ocean currents and Wind . . . . 33

3.3.1 Datasets . . . . . . . . . . . . . . . . 33

3.3.2 Ocean Currents: Mean and principal axes of variance . . . . . . . . . 34

3.3.3 Wind: Mean and Principal axes of variance . . . . . . . . . . . 37

3.3.4 Ocean Currents and Wind time series . . . . . . . . . . . . . 38

4 Processing of the HFR currents for the gridded Altimetry product 42

4.1 Introduction . . . . . . . . . . . . . . . . . . . 42

4.2 Estimating geostrophic currents from the HFR surface currents . . . . . . . . 42

4.2.1 Ekman Correction . . . . . . . . . . . . . . . . . 42

4.2.2 Optimal Interpolation to force geostrophy . . . . . . . . . . . . . . . 47

4.3 Comparison of Altimetry currents with various HFR processing . . . . . . 48

4.3.1 3-year time series of Current anomalies averaged over the 100-km wide coastal zone . . . . . . . . . . . . . . . . . . . 48

4.3.2 Spatial statistics using the OI and Ekman Corrected HFR method . . 49

4.3.3 Hovmöller diagram of OI and Ekman Corrected HFR and Altimeter

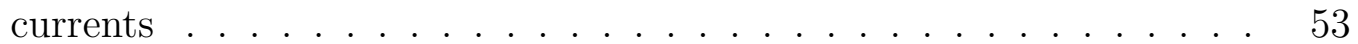


4.4 Processing HFR from currents to Sea Surface Heights . . . . . . . . . . . . . 54

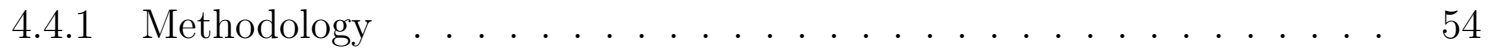

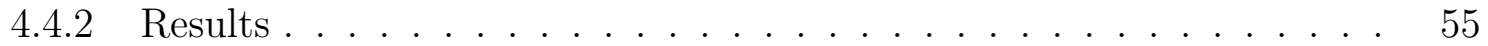

5 Methodology along an offshore altimeter track parallel to the coastline 57

5.1 Introduction . . . . . . . . . . . . . . . . . . 57

5.2 Altimeter Track Location . . . . . . . . . . . . . . . . . . . . 57

5.3 Data Processing . . . . . . . . . . . . . . . . . 58

5.3 .1 Processing of the altimeter data . . . . . . . . . . . . . 58

5.3 .2 Processing of the HFR currents . . . . . . . . . . . . . . . 59

5.4 Results . . . . . . . . . . . . . . . . . . . . . 62

5.4 .1 Along track SLA . . . . . . . . . . . . . . . 62

5.4 .2 Spectral Analysis . . . . . . . . . . . . . . . . 64

5.5 Statistics RMS/ Correlation . . . . . . . . . . . . . . . 65

5.5.1 Time Averaging choice for the HFR currents . . . . . . . . . . . . . . 67

5.6 Influence of the wind history . . . . . . . . . . . . . . . . . 68

5.6.1 Wind anomaly and quality of the HFR SLA in terms of RMS . . . 68

5.6.2 Concerned only with the across-track Ekman current . . . . . . . . . 69

5.6.3 Wind history: 4 general case studies . . . . . . . . . . . 71

5.7 Using an SST image to decide between HFR or Altimetry SLA as a closer

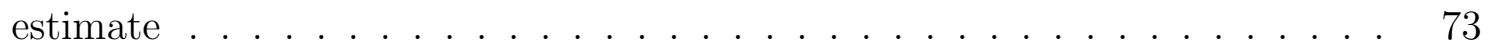

5.8 HFR and Altimetry across track velocity anomaly comparison . . . . . . . 75

5.9 Discussion-Overview . . . . . . . . . . . . . . . . . . 77

6 Methodology along altimeter tracks crossing the coastline $\quad 79$

6.1 Introduction . . . . . . . . . . . . . . . . . . . . 79

6.2 Motivation . . . . . . . . . . . . . . . . . . . . . . 79

6.3 Methodology ......................... 80 
6.4 Comparison over the open ocean with standard altimetry product . . . . . . 81

6.5 Comparison with PISTACH retrackers . . . . . . . . . . . . . . 81

6.6 Explaining some disagreement between HFR and altimetry . . . . . . . . . . 82

7 Applications $\quad 84$

7.1 Introduction . . . . . . . . . . . . . . . . . . . 84

7.2 Open Ocean . . . . . . . . . . . . . . . . . . . . 84

7.3 Near shore: case study Jason-2 P206 in the California Bight . . . . . . . . . 85

8 Conclusions $\quad 89$

$\begin{array}{ll}\text { Bibliography } & 93\end{array}$

\section{Appendix}

A JGR paper Roesler et al. 2013 


\section{Tables}

\section{Table}

2.1 The 3 color zones refer to the frequency-operating radar coverage; brown: 4 $\mathrm{MHz}$, blue: $12.5 \mathrm{MHz}$ and green: $25 \mathrm{Mhz}$. The table specifies their extent and spatial resolution. . . . . . . . . . . . . . . . .

4.1 Statistics for the 3-year time series of zonal $(\mathrm{u})$ and meridional (v) current anomalies averaged over the 100-km wide coastal zone, between altimetry and 4 processed HFR sets. . . . . . . . . . . . . . . . . . . . . 50

4.2 The 3-year mean correlation between altimetry and the various processed HFR current anomalies. The mean correlation for the zonal (u) and meridional (v) current anomalies is computed for the 54 collocated grid points. . . . . . .

4.3 The phase and magnitude of 3-year mean Complex correlation between altimetry and the various processed HFR current anomalies, computed for the 54 collocated grid points. . . . . . . . . . . . . . . .

5.1 RMS and correlation between the HFR/Altimeter SLA for various time-averaging of the HFR currents . . . . . . . . . . . . . . . . . 68 


\section{Figures}

\section{Figure}

1.1 U.S. west coast HFR and altimeter data set coverage. Green corresponds to the HFR 6-km spatial resolution while the red is the $2-\mathrm{km}$ resolution coverage. The blue lines are the ground tracks of the Jason-2 altimeter satellite. The dotted line is P206 for the interleaved Jason-1. . . . . . . . . . . . . . .

1.2 The number of $2-\mathrm{km}$ HFR vectors for the year 2010, are represented by the color background. Not all areas are sampled equally. The blue lines are the ground tracks of the Jason-2 altimeter satellite. The black lines are the ground tracks for the interleaved Jason-1. . . . . . . . . . . . . . . . . .

2.1 Interaction of a pulse of duration $\tau$ with a smooth sea surface; the illuminated surface geometry; and the resulting waveform . . . . . . . . . .

2.2 Jason-2 Ku Band Echo (in black). Brown waveform (in green) and parameters retrieved by MLE-4 . . . . . . . . . . . . . . . . .

2.3 (a) 20-Hz Waveforms from Jason-2 Cycle 26 pass 221, February 3 2008; (b) typical Brown Waveform; (c) Consecutive 20-Hz Waveforms, $30 \mathrm{~km}$ off shore in the presence of a Sig0-bloom event, over the region indicated by a black box in a) (d) other bloom waveform shapes at $21.18 \mathrm{~km}$ and $17.55 \mathrm{~km}$. (1) Brown Waveform, (2) increasing trailing edge, (3) Peakiness increased, (4) round pattern, (5) V-shape. . . . . . . . . . . . . . . . 
2.4 20-Hz Waveforms from J2 C026 Pass 221, within $10 \mathrm{~km}$ to shore: ocean to land transition. On the right, waveforms distorted by the presence of land,

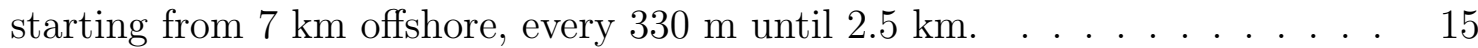

2.5 Left: Diagram showing the Bragg Scatter mechanism (from COCMP). Rigth: CODAR, compact HFR design, on Santa Catalina Island (from COCMP) .

2.6 A typical sea Echo Doppler spectrum. The sharp peaks around $\pm 0.5 \mathrm{~Hz}$ are due to the first order Bragg scatter. The frequency scales have been shifted so that the $25 \mathrm{MHz}$ radar operating frequency corresponds to $0 \mathrm{~Hz}$. The dash lines represent the expected position of the first order peaks in still water. There is a frequency shift of the peaks of $\triangle f=0.1 \mathrm{~Hz}$ which corresponds to

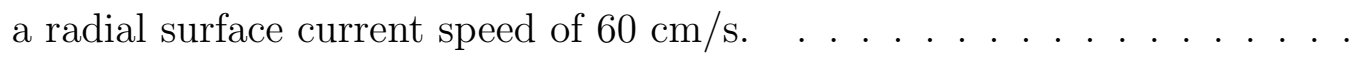

2.7 Map of the location of the HFR along the California coastline since 2009, as well as their coverage extent (Courtesy of COMCP). . . . . . . . . .

2.8 HFR geometry in Monterey Bay. Green dot: HFR radar sites. Red line: Baseline between two stations. Blue zone: part of HFR coverage area. Dotted line: radial line. Vector: radial surface current . . . . . . . . . . . . . . .

2.9 Mapping Error uncertainties provided with the gridded 6-km HFR surface currents, for (top) the zonal (u) and (bottom) meridional (v) component, on May 23, 2009, at two different times (Left) UTC 4:00 and (Right) UTC 6:00.

3.1 MODIS Terra SST image on Sep-25 2010, at UTC 18:50. It shows the narrow band of dark blue upwelled water next to the coast, stirred and distorted by all kinds of mesoscale eddies end jets. The black line is the Jason-1 interleaved

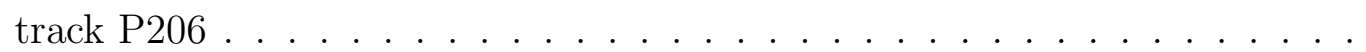

3.2 3-year mean (Right) and Principal Axis of Variance (Left) for HFR currents (top), Altimetry currents (middle), in the region where altimetry and HFR currents are coincident on a $25-\mathrm{km}$ resolution grid . . . . . . . . . . . . 36 
3.3 3-year mean (left) and Principal Axis of Variance (right) for the Winds, in the region where altimetry and HFR currents are coincident on a 25- $\mathrm{km}$ resolution grid . . . . . . . . . . . . . . . . . . . . 37

3.4 Meridional and zonal 3-year time series averaged over the 100-km wide coastal strip for HFR (in blue) and Altimetry (in red) currents . . . . . . . . . . . . 38

3.5 Meridional and zonal 3-year time series averaged over the $100-\mathrm{km}$ wide coastal strip (top) for HFR (in blue) and Altimetry (in red) currents anomalies; (middle) for the winds (in black). (bottom) 3-year time series of the EKE for the HFR (in blue) and altimetry (in red) currents and for the winds (in black) .

4.1 Residuals of the mean HFR currents minus mean Altimetry currents (in blue); 3-year mean wind tensor $\|W\| W$ (in red) on a $25 \mathrm{~km}$ resolution grid . . . . . 44

4.2 Estimates of $\varphi_{E}$ in degrees (left) and $\alpha_{E}$ (right) from the Least Squares . . . 45

4.3 3-year mean of the residuals HFR - Estimated Ekman currents (left) and 3-year mean Altimetry currents (right) . . . . . . . . . . . . . . . . . 45

4.4 3-year time series of Zonal current anomalies for various processing of the HFR currents. Top: HFR only, Middle: Ekman Corrected HFR, Bottom: OI Ekman Corrected HFR . . . . . . . . . . . . . . . . . . . . .

4.5 a) 3-year mean correlation in $\mathrm{u}$ and $\mathrm{v}$ between altimetry and OI Ekman Corrected HFR current anomalies. (b) 3-year mean correlation in $\mathrm{u}$ and $\mathrm{v}$ between altimetry HFR current anomalies. . . . . . . . . . . . . . . . . 52

4.6 3-year Mean Complex correlation between altimetry and OI Ekman Corrected HFR current anomalies (left) Phase Angle (right) Magnitude . . . . . . . . 52

4.7 3-year time series of zonal(left) and meridional (right) current anomalies for altimetry and OI Ekman Corrected HFR and their difference (ALT - HFR), along longitude $-122.33^{\circ} \ldots \ldots \ldots$. . . . . . . . . . . . . 54 
4.8 (Left) 3-year mean correlation in SLA between altimetry and OI Ekman Corrected HFR. (Right) 3-year time series of the SLA for altimetry and OI Ekman Corrected HFR and their difference (ALT - HFR), along longitude -122.33. ${ }^{\circ}$

5.1 6-km resolution HFR data coverage for the year 2010. The color indicates the number of HFR hourly samplings over the year. The white line is Jason-1 P206 in its interleaved orbit . . . . . . . . . . . . . . . 58

5.2 OI output. (Left) OI retrieved SSH around J1 P206. (Right) Corresponding 6-km OI output currents in black; HFR input currents in red, every other $6 \mathrm{~km} 61$

5.3 Altimeter and HFR sea levels along P206; Jason-1 SLA smoothed with a 45km low pass filter (green); Raw HFR SLA (red); OI Ekman Corrected HFR

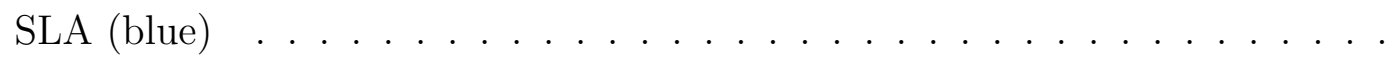

5.4 Seasonal variation of the altimeter SLA averaged along the Californian portion of P206 during 2010 . . . . . . . . . . . . . . . . . . . . 64

5.5 Spectrum for the Ekman Corrected HFR SLA (blue); for the Jason-1 at the 1-Hz level (black) and filtered with a boxcar window of $50 \mathrm{~km}$ (red) . . . . 66

5.6 Statistics for the 2010 Jason-1 cycles. STD of the altimeter SLA (blue). RMS between the raw HFR and altimeter SLA (dotted black). RMS between the Ekman Corrected HFR and altimeter SLA (red). Correlation between Ekman corrected HFR and altimeter SLA above 0.6 (red stars). Two cases are better correlated using the raw HFR SLA (black stars) . . . . . . . . . . . . 67

5.7 SLA and wind tensor anomaly Statistics for each 2010 Jason-1 Cycle. Top: mean averaged over the segment of the wind tensor anomaly magnitude (red) and direction (blue). (bottom) RMS between the raw HFR and altimeter SLA (dotted black). RMS between the Ekman Corrected HFR and altimeter SLA

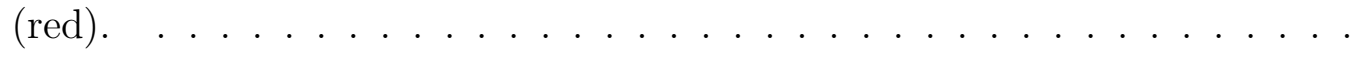


5.8 Case study for C326. (Left) Altimeter (green) and HFR (blue) SLA for (top) Raw HFR SLA (bottom) OI EKman Corrected HFR SLA. (Middle) Hourly wind speed along the altimter track. (Right) 3-day averaged wind tensor on P206 (black); Ekman Current (red) not to scale . . . . . . . . . . . . . 70

5.9 a) (Left) Daily winds centered at the time of passage for C311. (Right) 3-day (centered at the time of passage of the altimeter) mean and STD of the wind direction and magnitude along P206 for 2 similar cases C311 and C321. (b, c, d) Three consecutive daily winds, with the middle one centered at the time of passage of the altimeter. (Right) 3-day mean and STD of the wind direction and magnitude along P206. . . . . . . . . . . . . . . . . . . 73

5.10 (Left) SST image from the MODIS sensor on Terra on Jun 29, 2010 at UTC 19:40, 18 hours after the altimeter time of passage (Right) Altimeter and HFR sea levels along P206 for C312 on June 29. In green: The Jason-1 SLA are smoothed with a 45-km low pass filter; In red: the raw HFR SLA; In blue: The OI Ekman Corrected HFR SLA . . . . . . . . . . . . . . . . . . 74

5.11 Cross track Geostrophic velocities for 9 cycles in 2010: Altimeter (in green), OI Ekman Corrected HFR (in blue), 3-day averaged Ekman Corrected HFR surface currents projected across track P206 (in red) . . . . . . . . . . 76

7.1 Jason-1 C323 Sep 6: HFR ( blue) and altimetry (green) SLA. There is a rain event before latitude $36^{\circ} \mathrm{N} \ldots \ldots \ldots$. . . . . . . . . . . . 85

7.2 (Left) 2-km HFR ocean surface currents (in black) in the California Bight. Jason-2 P206 in red). (Right) Close up, between the two islands of Santa Rosa and Santa Cruz. . . . . . . . . . . . . . . . . . . 86 
7.3 (Left) Top: the 2010 mean of the altimeter MLE-4 SLA. Bottom: the P206 distance from a coastal feature. The coast is on the right side of the figure. (Right) Comparing altimeter and HFR SLA along P206. In blue: HFR SLA. In red: altimeter SLA after a boxcar filter of 91 points. In green: altimeter SLA after a boxcar filter of 21 points. The black dotted lines delineate the 10-km borders of the coastal features. The main coast is on the right side of the figures. (top) All the altimeter data is kept and the 2010 SLA mean is not removed. (middle) All the altimeter data is kept but the 2010 SLA mean is removed. (bottom) The extreme outliers are removed, and the 2010 SLA mean is removed . . . . . . . . . . . . . . . . . . . 87

7.4 Comparing altimeter and HFR SLA along P206. In blue: HFR SLA. In red: altimeter SLA after a boxcar filter of 91 points. In green: altimeter SLA after a boxcar filter of 21 points. The black dotted lines delineate the 10-km borders of the coastal features. The main coast is on the right side of the figures. . . 


\section{Chapter 1}

\section{Introduction}

\subsection{Introduction}

This chapter presents the motivation and scientific background behind the research presented in this dissertation. An overview of the prevailing methods developed for using altimetry in coastal regions and their validation is given. The major research goals are described. The study region and the data sets are presented. Finally the organization of the dissertation document is detailed at the end.

\section{$1.2 \quad$ Motivation}

Global climate change and its regional impact has become the focus of the global approach to research in recent decades. Climate and oceanic researchers consider that one of the most important roles played by the planet's oceans is the regulation of the Earth's

climate. In particular, the ocean is known to modulate atmospheric temperatures and carbon dioxide due to its much longer time constant for change than that of the atmosphere. Oceansurface currents strongly influence ocean-atmosphere interaction. The periodic ENSO (El Niño/Southern Oscillation) event, for example, is characterized by changes in surface currents is certain regions. Many marine organisms have their life cycle linked with ocean currents. The vast ocean plays an important role in shaping global climate on a rapidly changing planet. There is a need to observe, understand and model the diverse physical mechanisms that contribute to the ocean circulation to preserve this vast marine environment as well as 
to study its complex interaction with the Earth as a system.

The use of remote sensing devices on Earth-orbiting satellites since the 1970s developed by the American (NOAA, NASA) and European (CNES, ESA) spatial agency has revealed unseen aspects of the sea. This vantage point from space with its coverage, repeatability and sampling capabilities, provides new insights in ocean science and new ways of understanding the global ocean compared to the less dense in situ measurements. Measuring the sea surface heights (SSH), the ocean surface winds and the wave heights are a set of parameters needed for the study of the oceans and their evolutions. Satellite altimetry is one technique that estimates these parameters.

With over twenty years of experience conventional satellite altimetry is a mature technology over the open ocean. The conventional altimeters are of the type ERS-1 and 2, ENVISAT, TOPEX/Poseidon, Jason-1 and 2 and will be the focus of this thesis, in contrast to the most recently launched altimeters such as CRYOSAT and SARAL/Altika with new technologies. The basic altimetric measurement is the sea surface topography, its mounds and depressions, from which are derived the sea surface slopes and hence surface currents. With the correct constellation of satellites, multi-mission altimetry provides globally homogeneous, high resolution, and regular mapping of the mesoscale sea level and ocean circulation variations (Morrow and Le Traon 2006 [61]). The climate record over more than 20 years enables monitoring of, for example, the global mean sea level rise (Leuliette and Miller 2009 [56]) as well as the mesoscale eddy processes and their response to climate (Chelton et al. $2007[20])$.

Yet conventional altimetry and its application still face many challenges in coastal regions. This is an area where human interaction with the ocean is a maximum. The shelf regions have the most productive marine fisheries, they facilitate the development of oil and gas systems, they are the focus of marine recreation, and they are the location of a large number of search and rescue operations. With the increase of anthropogenic global climate change this zone is susceptible to anthropogenic stresses as well as natural hazards. 
Monitoring of the coastal ocean parameters can yield observations that can benefit various users of the sea, especially when the data are supplied in a timely way. These include information needed by mariners about waves, wind, and currents, or data for environmental quality managers concerning natural phenomena such as the occurrence of algal blooms or anthropogenic events like oil spills.

In coastal regions, the accuracy of the nadir-looking, pulse-limited satellite radar altimeter Sea Surface Height (SSH) measurement degrades. The geophysical (tides, dynamic atmospheric correction) and environmental (ionospheric, dry and wet tropospheric, sea state corrections) corrections, that need to be applied to the altimeter range, become less reliable and yet more variable (Andersen and Scharroo 2011 [2]) Secondly, the altimeter waveform (return echo) becomes distorted. Coastal waters differ from the open ocean due to rapid changes in bathymetry on the continental slopes, shallow waters and the presence of coastline boundaries. This induces greater variability resulting in shorter time and space scales. Possible rapid changes in sea state and/or the presence of land within the altimeter footprint affect the shape of the waveform. Deng et al. (2002, 2003 [26] [27]) observed that the waveforms from ERS-2 and TOPEX/Poseidon could be affected by land up to $20 \mathrm{~km}$ off the Australian coast. Furthermore waveforms can be degraded by the presence of unrealistic high radar return cross sections (Sig0) in the altimeter footprint, called Sig0-bloom events (Mitchum 2004 [60], Tournadre 2006 [73]). These Sig0-bloom events can occur from weak wind patches, as well as surface slicks, that create a highly reflective specular surface. These contaminated waveforms will not conform to the shape of the standard open-ocean Brown model formulated by Brown (1977 [14]). The ocean geophysical parameter (SSH, Significant Wave Height (SWH) and Sig0 related to surface wind speed) retrievals from these waveforms (retracking), fitted to the Brown model, will be unreliable.

Recovering these coastal altimetry data would be valuable for studies of the complex coastal circulation, sea level change and the impact on this coastal circulation. Some of the reasons are that the long-term altimetric measurements are repeatable, stable, and are the 
only long-term coastal measurements available in some remote areas. Altimetry data are also important for undrestanding the interaction between coastal currents and open ocean circulation, for mesoscale and coastal variability. At present, altimetry alone, even with corrected high-resolution along-track coastal data, will not resolve all the various time and spatial scales of coastal dynamics. The revisit time (10 days for Jason-2; a cycle) and the distances between tracks ( $\sim 200 \mathrm{~km}$ at 40 latitude for Jason-2) are too large, even with multiple altimeters. It must be considered as an important input to a coastal observing system. As such studies combining coastal altimetry and in situ data are adopted (Ruiz et al. 2009 [66], Le Hénaff et al. 2010 [50]). New regional coastal products are emerging and with them the prospect of exciting new applications. Developing tools for the generation and quality check of these coastal products is a pertinent area of research. About twenty years of archived altimeter data in the coastal zones are waiting to be reprocessed.

\subsection{Coastal altimetry background}

Current altimetry products use generic open-ocean processing which are retracked with the ocean Brown model and have been optimized for high precision along track 1-Hz (or 7 $\mathrm{km}$ ground resolution) SSH open-ocean variability. There is a loss of data in coastal regions from stringent quality checks (distorted waveforms, non-available corrections, ...) (Lee et al. 2009 [54]). The use of altimeter data in the coastal zone requires the development of new retrackers, applying improved local corrections and reprocessing techniques to increase data coverage (Cipollini et al. 2008 [21] , Bouffard. et al. 2008 [12]).

Some of the new strategies have recently been implemented and demonstrated their values (Birol et al. 2010 [11], Herbert et al. 2011 [38]). The COASTal ALTimetry (COASTALT) project (http://www.coastalt.eu) provides experimental coastal data for several regions in the European seas. The PISTACH (Prototype Innovant de Système de Traitement pour l'Altimétrie Côtière et l'Hydrologie) coastal product (PISTACH handbook 2010 [4]) is dedicated to the processing of Jason-2 altimeter data for the global coastal zone. But there 
are still many challenges to overcome for the full exploitation of coastal altimetry and their validation.

Improving the quality of altimeter geophysical retrievals is an important issue before using them for oceanographic applications. In this study we will focus on the effect of retracking. Retracking is a key element to extend the use of coastal altimetry (whether pulse-limited or the new Cryosat-2 SAR/InSAR technology) and produces improved results in shallow-water tide modeling and sea surface topography determination (Hwang and Chen 2000 [40], Deng and Featherstone 2006 [25]).

Over the last few years we have seen the development of new retrackers specific to coastal problems. Each has its own advantages and drawbacks. A review of waveform retracking methods can be found in Gommenginger et al. (2011 [34]. To optimize the choice of the retracking method waveform classifications are done (Deng and Featherstone 2006 [25]) and are even included as a data element (waveform class) in the PISTACH coastal product. However, there is a lack of a clear recommendation on which retracker to use depending on the situation. There is, also, a need to minimize the discontinuity of geophysical parameters from the open ocean to the coast. Changing retrackers from point to point along the track, depending only on the classification of the waveform, will create discontinuities from the relative biases between the various techniques. Consistency between the retrackers has to be investigated (Deng 2004 [24]).

The performance of retrackers can be estimated in a variety of ways. One possibility computes the statistics of residuals, using the geoid heights as a quasi-independent reference (Deng and Featherstone 2006 [25], Hwang et al. 2006 [41]). But the geoid may not be well resolved in the coastal regions. The validation of coastal altimetry data has been performed using tide gauges (Lebedev et al. 2011 [52]) for the SSH, moored ADCP for near surface geostrophic velocity and wave-rider buoys (Gómez-Enri et al. 2011 [33]) for the SWH measurements. The problem is that in these validations one compares sparse point measurements that may not lie exactly over the satellite track, in a highly dynamic 
region. High-Frequency Radar (HFR) radial velocities perpendicular to the satellite track were used to estimate the quality of the altimeter-derived velocity. The geometry of the HFR configuration, however, limits the number of collocations (Liu et al. 2012 [58]).

This thesis presents a novel approach to independently validate the coastal retrackers, using the HFR sea surface information that extends up to $150 \mathrm{~km}$ offshore along a continuous altimeter track and at the time of passage of the altimeter. The optimal method for altimetry retracking may turn out to be a combination of different retrackers for different parts of an altimeter track (Deng and Featherstone 2006 [25]) or it may change for different cycles on the same track. The HFR data will ensure a continuity of the corrected altimetric SSH from the open ocean to the coast. These relationships will be explored in the west coast of the U.S. over the years 2008 to 2010, where the coverage of HFR surface current is excellent, with resolutions of $2 \mathrm{~km}$ and $6 \mathrm{~km}$ depending on HFR operating frequency (Fig. 1.1).

Previous studies have compared altimetry and HFR surface current maps that support the potential of our methodology. Saracenco et al. (2008 [67]) show good correlations between a yearly time series of HFR velocities and an improved coastal SSH product at three locations along the Oregon coast. Two studies confirm that HFR contain more submesoscale information (Chavanne and Klein 2010 [17], Kim et al. 2011 [47]] than present-day satellite altimetry. Consequently, the 2-km HFR data can help assess the feasibility of creating a higher resolution coastal product by exploiting the higher frequency $20-\mathrm{Hz}$ (or $\sim 330 \mathrm{~m}$ ground resolution) altimetric range rate measurements and implementing new editing and filtering techniques. This enhanced resolution coastal data set will better resolve the smaller scale of oceanographic processes in coastal zones. Even without land contamination in the altimeter footprint, the waveforms may be degraded by non-homogeneous ocean dynamics or the occurrence of unusually high radar return backscattering cross section. We will explore the possibility of exploiting the HFR sea surface information that extends up to $150 \mathrm{~km}$ offshore to correct for these effects as well.

The main innovative research objective of this thesis is to answer how can the HFR cur- 
rents be exploited to correct for conventional satellite altimetry over the continental shelves and the coastal regions. The HFR set could enable a systematic validation of altimetry retrievals along the continental shelf at the time of passage of the altimeter. It could also provide steps to understand the behavior of the waveforms and impact on the choice of retrackers, and might offer helpful insights on how to evaluate other corrections included in the altimeter SSH.

\section{$1.4 \quad$ Objectives}

(1) To define a robust methodology to compare HF coastal radars and altimetry measurements. This methodology will be explored first in the offshore regions, when the altimeter data are reliable, at the large-scale level, then along an altimeter track.

(2) HFRs main measurements are surface currents, whereas it is SSH for altimetry. Should we compare along-track SSHs or cross-track currents? What information gain could we get from one or the other method?

(3) No measurement system is perfect. The physical content and limitations of each dataset needs to be understood, and a consistent way to compare them developed.

(4) What conditions are required to get a good fit between the two sets? Does it depend on the seasons, the structure of the currents or the winds?

(5) To investigate how we can use HF coastal radars to correct the problems related to altimetry and get some insight on the causes.

(6) In the coastal region, explore the use of HFR currents to validate several retrackers.

(7) In the coastal region, explore the use of the 2-km resolution HFR currents to refine the spatial resolution of the altimeter data using the $20-\mathrm{Hz}$ measurements. 


\subsection{Data sets}

\subsubsection{High-frequency coastal radars}

HFR surface current maps spanning the entire California and Oregon coastline have been available since August 2008 at a $6-\mathrm{km}$ resolution. On the U.S West coast we have collected the HFR network coastal current set generated by Kim et al. (2008 [46]) at the Scripps Institution of Oceanography. They provide hourly ocean surface currents resampled and post-processed to $6-\mathrm{km}$ and $2-\mathrm{km}$ gridded map products from the combined radials with a range of $150 \mathrm{~km}$ and $50 \mathrm{~km}$ offshore respectively (Fig. 1.1), as well as their uncertainty. We have data sets from 2008 till 2010. Their 2-km resolution grids became available since 2009 in some selected regions and is extending as the network increases (Fig. 1.2).

\subsubsection{Altimeters}

We will focus on Jason-1 and Jason-2 (launched in July 2008) altimeters that were coincident (July 08- Feb 09) then interleaved from February 2009 until March 2012. After March 2012, Jason-1 has been placed in a geodetic orbit. The advantage of Jason-2 is that its performance is improved in coastal region, due to several technical innovations. Also the Jason-2 PISTACH level-2 product includes several retrackers and dedicated coastal corrections. The region of data coverage offer distinct, more or less challenging zones, with and without islands; with some track transitioning from ocean to land (P221) and from land to ocean (J2 P206) (Fig. 1.2).

\subsection{Dissertation Overview}

The remainder of this document is organized as follows:

Chapter 2 describes the Satellite altimetry and HFR measurements as well as the datasets. 


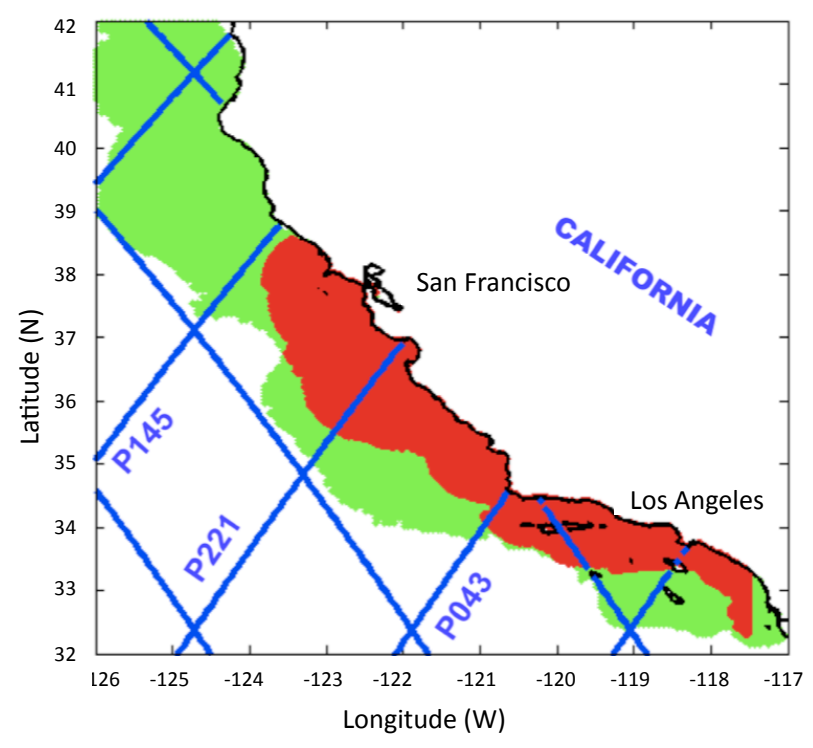

Figure 1.1: U.S. west coast HFR and altimeter data set coverage. Green corresponds to the HFR $6-\mathrm{km}$ spatial resolution while the red is the $2-\mathrm{km}$ resolution coverage. The blue lines are the ground tracks of the Jason-2 altimeter satellite. The dotted line is P206 for the interleaved Jason-1.

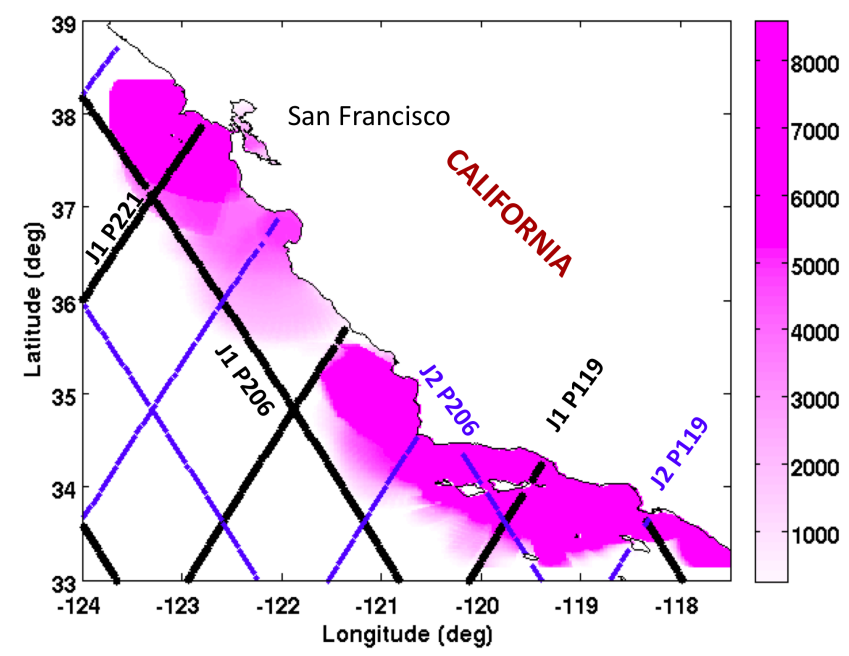

Figure 1.2: The number of 2-km HFR vectors for the year 2010, are represented by the color background. Not all areas are sampled equally. The blue lines are the ground tracks of the Jason-2 altimeter satellite. The black lines are the ground tracks for the interleaved Jason-1. 
Chapter 3 provides the mesoscales characteristics of the Altimeter and HFR derived velocity observation in the California Current Systems.

Chapter 4 studies the large mesoscale relationship between the altimeter and HFR data along a 100-km wide coastal strip. It details the methodology for processing the HFR surface currents to make them comparable with altimeter currents and sea level measurements for a weekly and $30 \mathrm{~km}$ gridded resolution product.

Chapter 5 presents the HFR data processing along a descending offshore altimeter track, where the altimeter data is not contaminated by the coastal problems. This altimeter track is parallel to the coastline and provides $750 \mathrm{~km}$ of along track data coincident with the 6-km resolution HFR currents.

Chapter 6 details the HFR data processing along the ascending tracks that transition from the ocean to land. There is about $100 \mathrm{~km}$ of along track data coincident with the 6-km and/or 2-km HFR product closer to shore. It tests the feasibility of retrieving a 2-km resolution altimeter product closer to shore, and compares several retrackers.

Chapter 7 presents some applications.

Chapter 8 summarizes the conclusions. 


\section{Chapter 2}

\section{Satellite Altimeter and HFR measurements and Data sets}

This chapter describes the distinct radar technique involved for the satellite radar altimeter and the HFR to remotely sense the ocean surface, the methods to extract their main ocean surface geophysical measurements from the signal, as well as error sources in their estimates. This section also specifies the datasets that are used in this study.

\subsection{Satellite Altimetry}

\subsubsection{Concept}

With more than 20 years of experience since the launch of Geosat (1985) and the following successive missions including ERS-1-2, Envisat, TOPEX/Poseidon, and Jason-1-2, satellite radar altimetry data has become a standard tool for studying the ocean circulation. The satellite altimeter measures the range to the ocean's surface via the two-way time travel between the emission of the radar pulse and the reception of the echo. The difference between the altitude of the orbit above a reference ellipsoid and the range corrected for various instrumental, propagation and environmental effects provides the SSH above the reference ellipsoid. The accuracy required for oceanographic studies is of a few centimeters.

The Jason type satellite altimeters are dual frequencies $(\mathrm{Ku}(13.5 \mathrm{GHz})$ and $\mathrm{C}(5.3$ $\mathrm{GHz}$ ) bands) nadir-pointing instruments that emit short pulses reflected by the sea surface. The geometry of the footprint is pulse-limited. Pulse compression based on the transmission of a chirp, a linear time frequency modulated, radar impulsion, is used to achieve high 
accuracy ranging. An onboard Fast Fourier Transform (FFT) generates an echo spreading over 128 range gates, separated by about $47 \mathrm{~cm}$. This is the intrinsic range resolution of the altimeter. The time evolution of the echo, the waveform, represents the mean return backscattered power as a function of time. To reduce speckle the individual return echoes are averaged, on-board, typically over 100 successive echoes (at Ku-band) over a period of $50 \mathrm{~ms}$. These $20-\mathrm{Hz}$ waveforms are transmitted to the ground where retracking is applied to refine the extraction of the oceanic parameters, which will lead to the ultimate range accuracy. For open ocean generic products, the data are averaged to 1-Hz (Chelton et al. 2001 [19]) the range accuracy is $1.5 \mathrm{~cm}$ for a SWH of $2 \mathrm{~m}$, and the orbit accuracy is at the $1 \mathrm{~cm}$ level for Jason-1-2.

Over water, after the pulse hits the surface, the illuminated surface area grows from a point to a disk, and then spreads as an annulus, increasing in diameter but with a constant surface area. The corresponding waveform shows a characteristic shape with a sharp rise to a maximum level (leading edge), followed by a gradually sloping trailing edge, as the off-nadir signal slowly reaches the edge of the radar beam (Fig. 2.1). The waveform gives information about the ocean surface. The waveform provides the range between the satellite and the surface at nadir via the two-way travel time of the transmitted pulse, the Significant Wave Height (SWH) via the slope of the leading edge, and the backscattering coefficient (Sig0 pronounced 'sigma naught') which represents the surface roughness via the returned power (Fig. 2.2). This ocean-shape can be represented by an analytical Brown model (Brown 1977 [14]). Jason-1 and Jason-2 waveforms are ground-retracked using a Maximum Likelihood Estimator (MLE) fit to the Brown model. The MLE-3 retrieves 3 geophysical parameters (range, SWH, and Sig0), the MLE-4 also estimates the antenna mispointing angle (slope of the trailing edge) (Amarouche et al. 2004 [1]) 


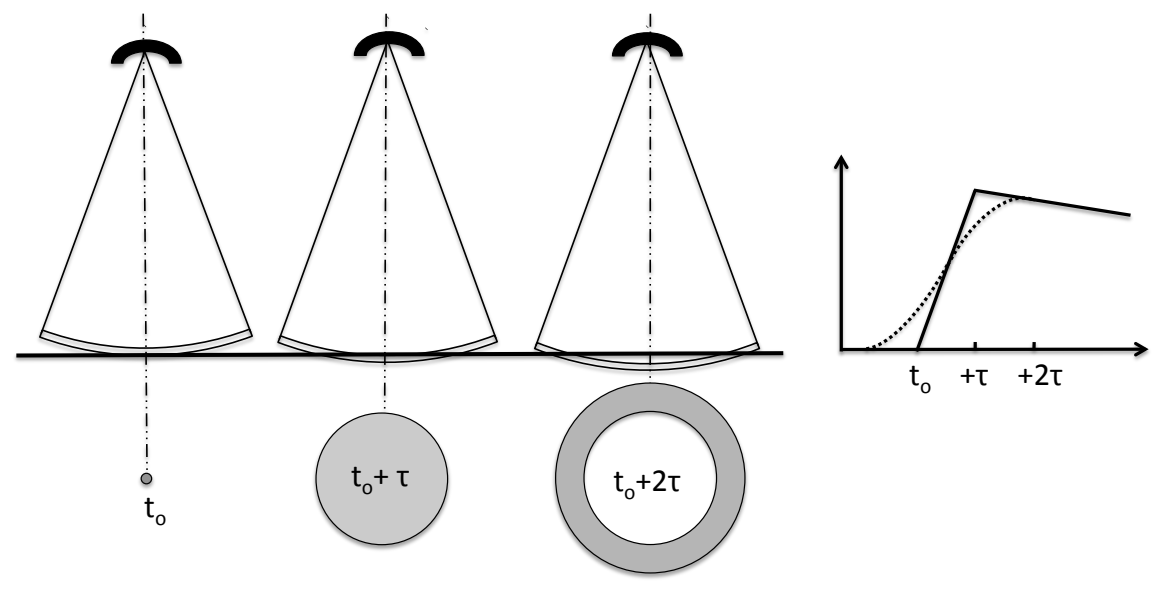

Figure 2.1: Interaction of a pulse of duration $\tau$ with a smooth sea surface; the illuminated surface geometry; and the resulting waveform

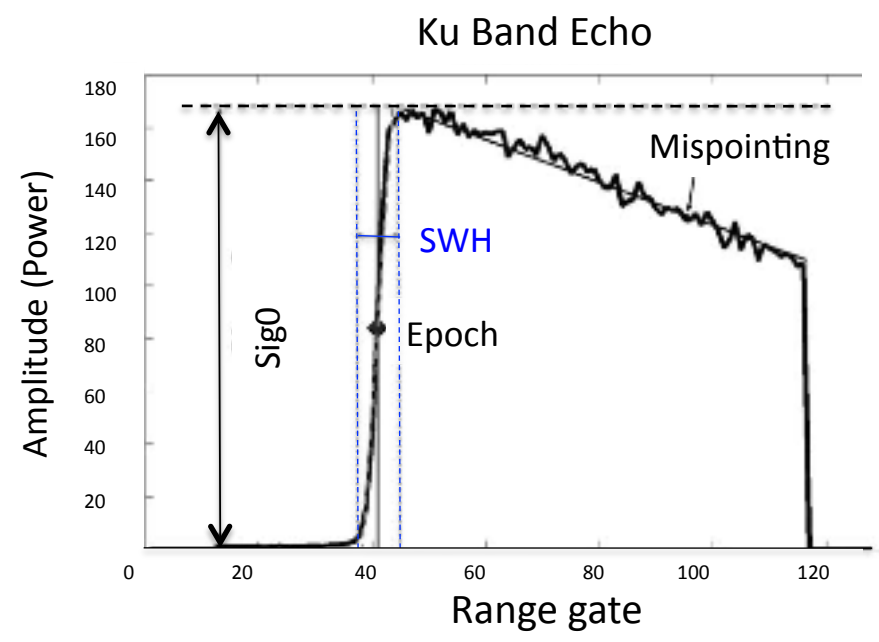

Figure 2.2: Jason-2 Ku Band Echo (in black). Brown waveform (in green) and parameters retrieved by MLE-4

\subsubsection{Waveform contamination}

The Brown model has been derived from the physical properties of a rough and homogeneous scattering surface, for near normal incidence. It is based on physical optics theory where the surface is treated as a set of specular facets with a given height and slope prob- 
ability density function. The waveform can be expressed as a convolution of three terms, the average impulse function from a flat surface, the surface elevation probability density function of specular points within the altimeter footprint and the radar system point target response (Brown 1977 [14], Hayne 1980 [35]). Although different refined analytical forms exist, one of the main assumptions related to the surface properties (not the instrument, or pulse shape) are that the sea surface is homogeneous over the footprint and that the Probability Distribution Function (PDF) of the surface slope and elevation has a pre-defined shape, essentially Gaussian. When this is not the case, the waveform will not conform to the Brown model, and new retracking strategies will need to be implemented.

The altimeter waveforms may be corrupted by non-uniform radar return backscattering cross section Sig0 in the altimeter footprint. In the occurrence of unusually high Sig0 due to highly reflecting ocean patches, these events are called Sig0 blooms (Mitchum 2004 [60], Tournadre 2006 [73]). Sig0 blooms could occur in regions of weak winds, in the presence of surface slicks, and possibly other geophysical coastal conditions. They can create various waveform shapes (Fig. 2.3).

For an ocean to land transition, the altimeter footprint will gradually contain more and more land returns (Fig. 2.4). First the trailing edge part will be perturbed by land; then more waveforms samples become affected by the land reflections, moving towards the leading edge. The shape of the coastline, the relief, the backscattering properties of the terrain will produce a variety of coastal waveform shapes. On a same location, they could change depending on the season, or the position of the satellite track, which can deviate +/$1 \mathrm{~km}$ from the nominal track. In addition to land return effects, calm water surfaces near the coastline, such a as bays or estuaries, make the return power higher and narrower, thus contaminating the waveform as well.

Retracking is a procedure of waveform data post processing to improve parameter estimates over those given by the generic open ocean product. Waveform retracking has different emphasis for different applications depending on the reflecting surfaces. 
a)

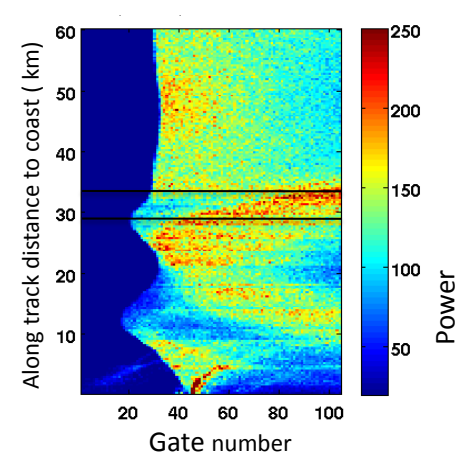

b)

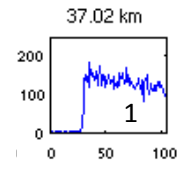

c)

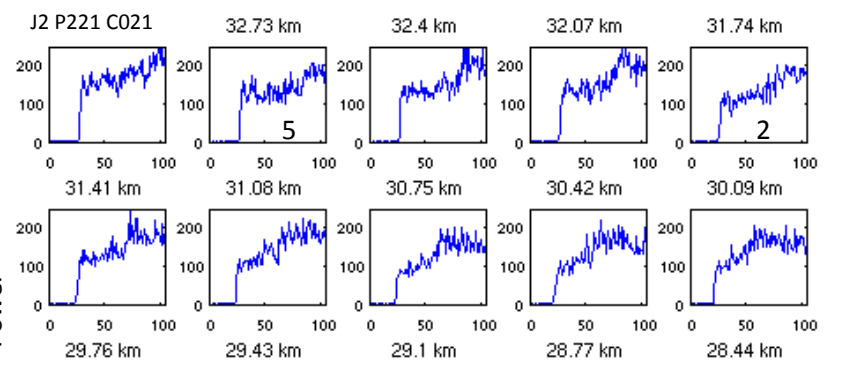

d)
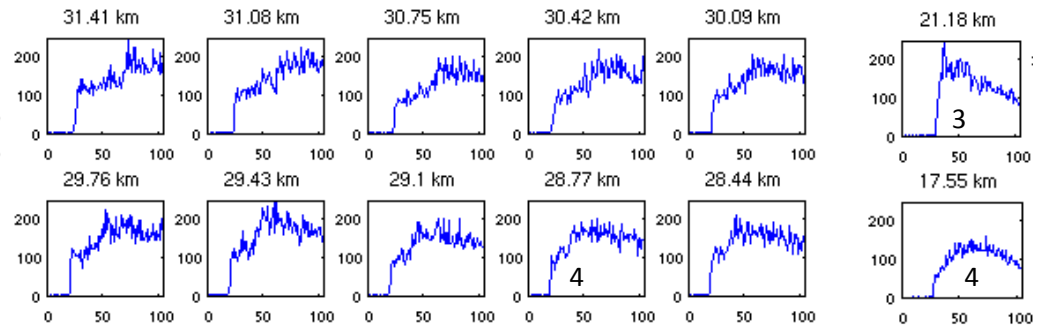

Figure 2.3: (a) 20-Hz Waveforms from Jason-2 Cycle 26 pass 221, February 3 2008; (b) typical Brown Waveform; (c) Consecutive 20-Hz Waveforms, $30 \mathrm{~km}$ off shore in the presence of a Sig0-bloom event, over the region indicated by a black box in a) (d) other bloom waveform shapes at $21.18 \mathrm{~km}$ and $17.55 \mathrm{~km}$. (1) Brown Waveform, (2) increasing trailing edge, (3) Peakiness increased, (4) round pattern, (5) V-shape.
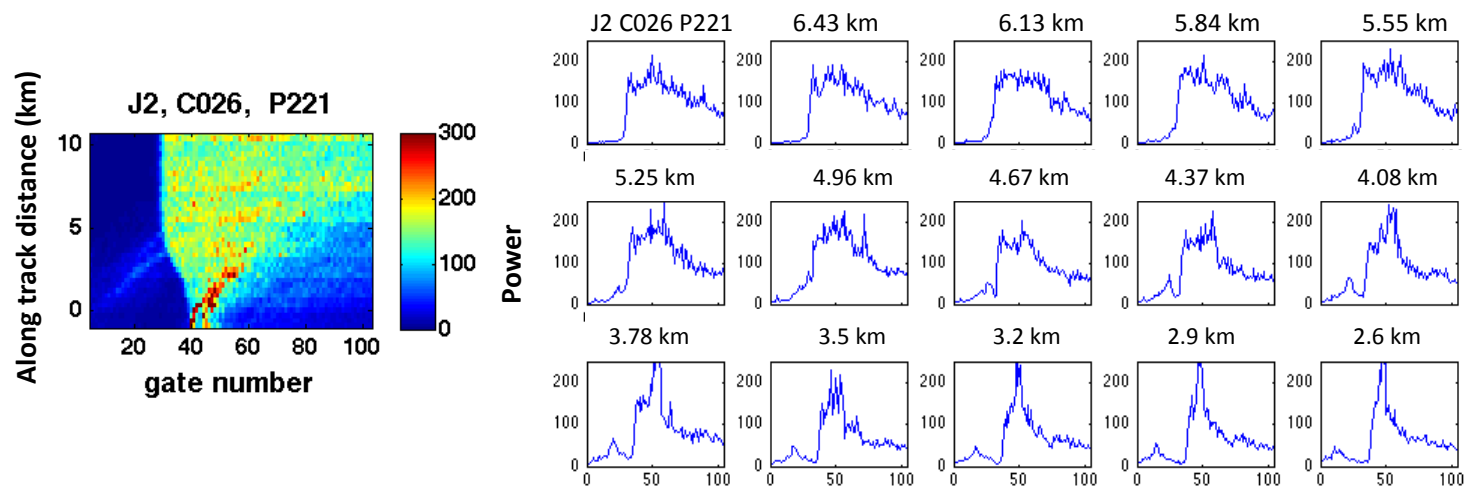

Figure 2.4: 20-Hz Waveforms from J2 C026 Pass 221, within $10 \mathrm{~km}$ to shore: ocean to land transition. On the right, waveforms distorted by the presence of land, starting from $7 \mathrm{~km}$ offshore, every $330 \mathrm{~m}$ until $2.5 \mathrm{~km}$. 


\subsubsection{Altimetric sea-level height corrections}

Retracking improves the estimate of the range, but as the radar signal does not travel in vacuum it must be adjusted for propagation effects through the atmosphere (dry and wet) and the ionosphere. The ionospheric delay is proportional to the square of the wave frequency, and is corrected based on the range difference between the $\mathrm{Ku}$ and $\mathrm{C}$ band. The very variable wet tropospheric delay is estimated by the onboard radiometer instrument. The range must also be corrected from on electromagnetic bias, the Sea State Bias (SSB), which is the difference between the true sea surface and the one seen by the radar depending on sea state conditions. Over the open ocean the final sea surface height with respect to a reference ellipsoid has an accuracy of about $4 \mathrm{~cm}$ averaged over 1 second (Chelton et al. $2001[19])$.

However these corrections can be problematic in the littoral regions. The Jason type altimeters carry onboard Microwave Radiometers (MRs) to correct for the water vapor, but close to shore the MR footprint will be contaminated by land. Due to technical improvements the Advance Microwave Radiometer (AMR), onboard Jason-2, water vapor estimates are probably not corrupted by land until $25 \mathrm{~km}$ offshore relative to $50 \mathrm{~km}$ for the Jason-1 MR. For the highly variable in time and space water vapor corrections, different strategies exist such as correcting the altimeter radiometer due to land contamination (Desportes et al. 2007 [28], Brown 2010 [15]). The frequency dependent ionospheric path delay is calculated from the dual-frequency altimeter (C-band and $\mathrm{Ku}$-band); but land also contaminates their footprints (C-band has a larger beam width). The ionospheric correction Global Ionospheric Map (GIM) derived from the Global Positioning System (GPS) network is recommended in coastal areas. The Sea State Bias (SSB) correction compensates for the bias of the altimeter range measurement toward the troughs of the ocean waves, as well as for an instrumental bias. It depends on the sea state (wave types and wind field). The open ocean SSB is empirically determined from the Significant Wave Height (SWH) and the wind speed (Tran et al. 2010 
[74]. In the coastal zone, with complex wind and wave dynamics, this empirical relationship may not be valid. Depending on the application, the SSB will or not be included. Finally once the range has been corrected, the SLA is computed relative to a Mean Sea Surface (MSS) level:

$$
\begin{gathered}
\text { SLA S Satellite height }- \text { Corrected range - MSS - Tides height } \\
- \text { Atmospheric pressure loading }
\end{gathered}
$$

where

$$
\text { Corrected range }=\text { Altimeter Range }+ \text { Atmospheric corrections }+ \text { SSB }
$$

Other important considerations in the coastal regions are the tidal and HF atmospheric pressure loading corrections that are less accurate. All of the aforementioned environmental corrections are subjects of ongoing research. Depending on the altimeter data set used and the focus of the analysis (more on the open ocean or on the littoral regions) some improved corrections will, or will not, be implemented. It is important to acknowledge these problems, so that we understand that the altimeter segments could be affected by these corrections and, each one of them, in variable amounts and at variable distances from the shoreline.

\subsubsection{Datasets}

Several altimeter data sets all distributed by AVISO are used. The first one is the weekly multi-mission altimetry Merged Sea Level Anomaly (MSLA) product ((M)SLA handbook 2013 [6]), gridded sea surface heights computed with respect to a seven-year mean, on a 1/3 x 1/3 Mercator grid. This product combines data from different missions. More specifically, we use the Delayed-Time, Updated series. This data set usually has no valid values for off shore distances closer than about $30 \mathrm{~km}$, where the along track altimeter data has been flagged bad due to its proximity to land. 
The second set is the global delayed-time along-track Sea Level Anomalies (SLA) product (DT SLA handbook 2012 [5]), which provides standard open-ocean 1-Hz (ground track spacing of $\sim 6 \mathrm{~km}$ ) along-track sea level anomalies computed with respect to a seven-year mean, with all standard corrections already applied. For the previous two sets, the corrections applied to the sea surface heights are from the regular open ocean processing; no specific coastal corrections have been applied.

The third set is the Sensor Geophysical Data Record (SDGR) along track product. They contain the MLE-4 at the $1-\mathrm{Hz}$ and $20-\mathrm{Hz}$ data rates as well as the $20-\mathrm{Hz}$ waveforms and the information about the tracker.

The last set is the Jason-2 PISTACH coastal product [PISTACH handbook 2010 [4]]. For each correction affected by the proximity of land (such as the wet tropospheric correction) it offers a varied choice of correction scenarios. PISTACH also gives output for three new retracking schemes at the $20-\mathrm{Hz}$ rate: Oce3, Red3 and Ice3. These three specific PISTACH retrackers will be analyzed together with one of the conventional Jason-2 deep-ocean retracker MLE-4. The Oce3 retracker represents the output of MLE-3 performed on a de-noised waveform, filtered after a Singular Value Decomposition (SVD) (Severini J. 2010 [68]). The Red3 retracker also uses MLE-3 but is done on a restricted analysis window around the leading edge, to remove the eventual gates corrupted by the effects of land. The Ice3 retracker is a 30\% threshold method (Davis 1997 [23]), also implemented on a restricted analysis window. The PISTACH sea level anomalies will be computed from the 20-Hz data stream for the various retrackers.

The processing of the several data sets will be explained in more details for each chapter as they depend on the application.

\section{$2.2 \quad$ HFR}

High Frequency Radar (HFR) ocean systems provide vector maps of currents in the coastal region. It is a land-based system that remotely scans the sea surface day and night 
in all weather conditions at a rapid rate (minutes) and close spatial interval (kilometers). The measurements are unique in their proximity to the ocean surface and the extent of the coastal coverage. While conventional in-situ measurements only provide sparse singlepoint measurements at a great distance from one another, HFR provide 2 dimensional maps of oceanic flow over a much larger area. This relatively new operational technology in oceanography produces real time results available to the scientific community. The studies and applications of coastal ocean currents based on HFR have recently flourished (Paduan et al. 1996 [63], Kim et al. 2010 [45], Gurgel et al. 2011 [36]). The evaluation and limitation of the measurements are on ongoing process.

Radio oceanography was developed during World War II in order to provide ship, aircrafts and even submarine locations. Sea echoes from the ocean were then undesirable. But an unexpected application was discovered. The sea clutter at the HF has a large and well-defined return in the Doppler Spectrum. It caught the interest of Crombie (1955 [22]) who established the relationship between the backscatter of coherent HFR, operating at frequencies 3-30 MHz, and wave trains on the ocean surface. Barrick, Evans and Weber (Barrick et al., 1977 [7]) were among the first to exploit this phenomenon for the extraction of geophysical information about ocean surface currents and ocean wave directional spectrum.

\subsubsection{Concept}

A transmitted radio wave is scattered from any surface of the ocean with which it interacts. The radar detects the sea backscatter and measures the time and phase difference between the transmitted and received waves (Fig. 2.5). The time signal is converted into the frequency domain using an FFT. The resulting power spectrum is referred to as the Sea echo Doppler spectrum and is essential to extract the ocean surface information such as currents and waves. In particular, the sea echo Doppler spectrum at HF (Fig. 2.6) is generally dominated by two very sharp peaks above and below the transmit frequency. These welldefined peaks enable the extraction of surface currents. It was deduced by Crombie (Crombie, 
1955 [22]) that these peaks in the spectrum is a form of Bragg scattering (same phenomenon responsible for scatter of X-rays in crystals), resulting from sea surface wave trains lining up to form a diffraction grating for the radio waves. The Bragg resonant ocean waves are those having a wavelength of exactly half the radar wavelength, so that the backscattered coherent radio waves will interfere constructively on the receiver. The two prominent peaks, symmetrically spaced about the carrier frequency, correspond to ocean waves propagating directly toward and away from the radar. For the HF band, the corresponding wavelengths of the Bragg resonant ocean waves are 5 to $50 \mathrm{~m}$. The range of Bragg resonant ocean wave frequencies is about 0.2 to $0.6 \mathrm{~Hz}$ and the phase speeds range from about 3 to $10 \mathrm{~m} / \mathrm{s}$. In many of the coastal marine environments where HFR systems are deployed, these Bragg waves are propagating in all directions and have high enough amplitudes to result in a strong sea echo nearly continuously.
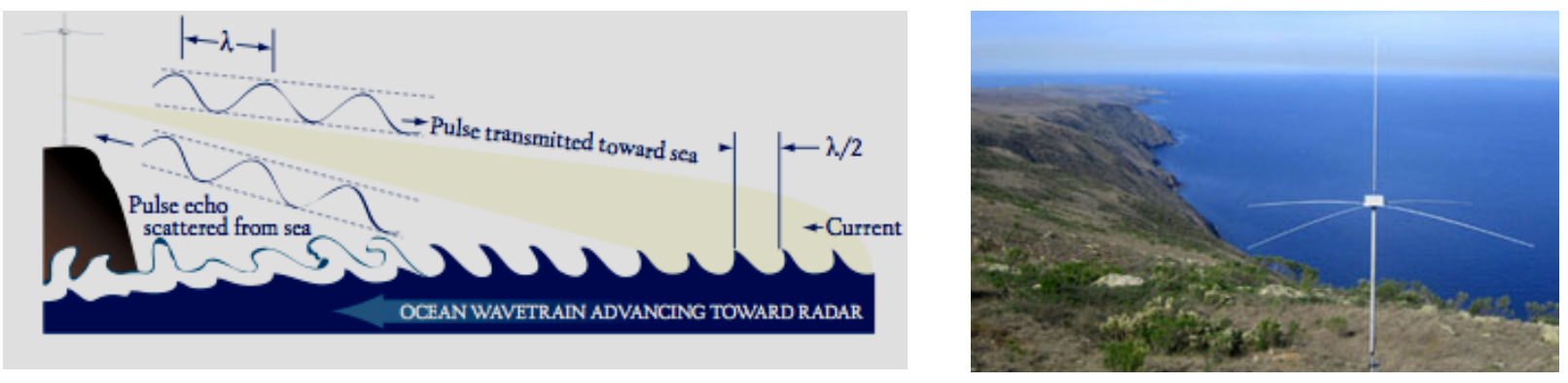

Figure 2.5: Left: Diagram showing the Bragg Scatter mechanism (from COCMP). Rigth: CODAR, compact HFR design, on Santa Catalina Island (from COCMP)

\subsubsection{Sea Doppler Spectrum and sea surface current measurements}

Fig. 2.6 is a typical HFR Doppler Spectrum. In the figure two strong peaks are seen at about $\pm 0.5 \mathrm{~Hz}$, one is due to the wave propagating toward the radar (positive peak), the other away (negative peak) and are due to the first order Bragg scatter. The two sharp Doppler peaks caused by the celerity of the resonant Bragg waves relative to the radar. This celerity is the sum of two components: the sea surface current over which the waves 


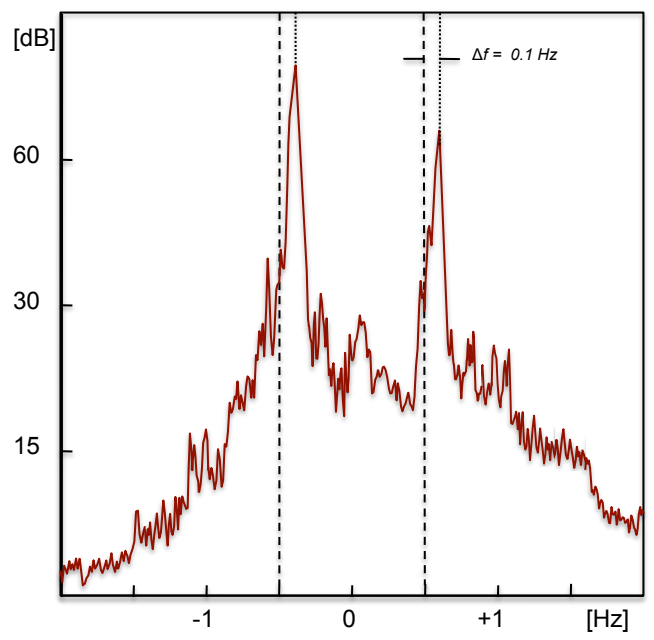

Figure 2.6: A typical sea Echo Doppler spectrum. The sharp peaks around $\pm 0.5 \mathrm{~Hz}$ are due to the first order Bragg scatter. The frequency scales have been shifted so that the 25 $\mathrm{MHz}$ radar operating frequency corresponds to $0 \mathrm{~Hz}$. The dash lines represent the expected position of the first order peaks in still water. There is a frequency shift of the peaks of $\triangle f=0.1 \mathrm{~Hz}$ which corresponds to a radial surface current speed of $60 \mathrm{~cm} / \mathrm{s}$.

are travelling and the still water phase velocity of the wave trains. The wave trains have wavelengths of the order of 1 meter, and are gravity waves (restoring force gravity) as

opposed to the much smaller capillarity waves (restoring force surface tension). The phase velocity of gravity waves propagating in the deep ocean (depths greater than about half the wavelength) is well known: $c_{p}=\sqrt{\frac{2 \pi g}{\lambda}}$ where $\lambda$ is the wavelength of the ocean wave and $g$ is the acceleration due to gravity. The Doppler shift for the still water phase speed is: $\triangle f=\frac{2 c_{p}}{\lambda_{r}}$, where $\lambda_{r}$ is the wavelength of the transmitted radar signal. Bragg resonance occur when $\lambda=\frac{\lambda_{r}}{2}$ (this is true for collocated transmit and receive antennas), so that $\triangle f=\sqrt{\frac{g}{\pi \lambda_{r}}}$ and depends only on the frequency of the radar. Deviations from the expected Bragg Doppler shift can be attributed to the radial component of the surface current relative to the radar. Only the resonant sea waves that are travelling radially away or toward the radar contribute to the energy of the first order spectrum. These narrow peaks are not sensitive to the sea state. The higher order sea echo spectrum varies with sea state, and includes secondary peaks and a continuum around the first order spikes. Their distribution varies with the wave 
directional spectrum and their amplitude with the SWH. Two independent second order effects contribute to this continuum. One component corresponds to radar waves scattered twice from ocean waves. The other corresponds to nonlinear surface waves that interact to satisfy the Bragg wavelength. These are highly sensitive to the sea state and manifest as a natural broadening of the first order peak without affecting their position, and do not perturb the current measurements from the Doppler peaks, except in the limit of very high SWH $(\mathrm{SWH}>5.4 \mathrm{~m}$ for a $12.5 \mathrm{MHz}$ system).

\subsubsection{HFR Systems}

The first commercial HF ocean radar for the measurement of ocean surface properties was the Coastal Dynamic Application Radar (CODAR), and then upgraded to the SeaSonde, a compact commercial radar built by CODAR Ocean Sensors, Ltd California (Fig. 2.5). Other systems have been developed, such as the Ocean Surface Current Radar (OSCR) in the United Kingdom and the Wellen Radar (WERA) in Germany. The HF radio wavelengths span the range 10-100 m. With Conventional radar design, angular resolution is determined by the width of the radio signal beam, which is proportional to the radar wavelength and inversely proportional to the antenna size. To obtain good resolution requires huge antennas: a $5^{\circ}$ angular resolution demands an $\mathrm{HF}$ receive antenna that is $100-1000 \mathrm{~m}$ in length and would be difficult to steer. However it is only at the HF that the Sea Echo Doppler spectrum relates neatly to the ocean currents and waves. To solve this problem two methods for angular resolution exit: one is Direction Finding (DF) and is implemented by the CODAR system, the other is Beam Forming used by linear phased array systems (WERA, OSCR). Both have advantages and disadvantages. One advantage of Beam Forming is that the beam can be steered electronically to a particular angle. But these systems are still large, and difficult to place on a line along the coastline. For example, OSCR comprises a linear array of 16 receiving antennas spanning 85-m long. On the other hand DF enables the use of collocated antenna, which requires a sophisticated algorithm to solve for the incoming direction. 
HF ocean radar emits vertical polarization. Firstly, the average ocean vertical polarization radar cross section per unit area is nearly independent of the incidence angle near $30^{\circ}$ of the grazing angle, unlike the horizontal polarization radar cross section, which is, anyway, at least $30 \mathrm{~dB}$ lower than the vertical component. Secondly, HF radars are in groundwave propagation mode, which means that the radar signal follows the surface of the sea. This method only works for a vertically polarized signal in contact with the salty conductive water.

In 2002, the coastal Ocean Currents Monitoring Program (COCMP) began creating a California network of HFR instrument to monitor coastal ocean surface currents in real time and provide surface current forecasting capability. In 2009 there were over 54 landbased stations spanning the California coastline. The CODAR system is prevalent on the California coast due to the limited space needed for its deployment (Fig. 2.7).

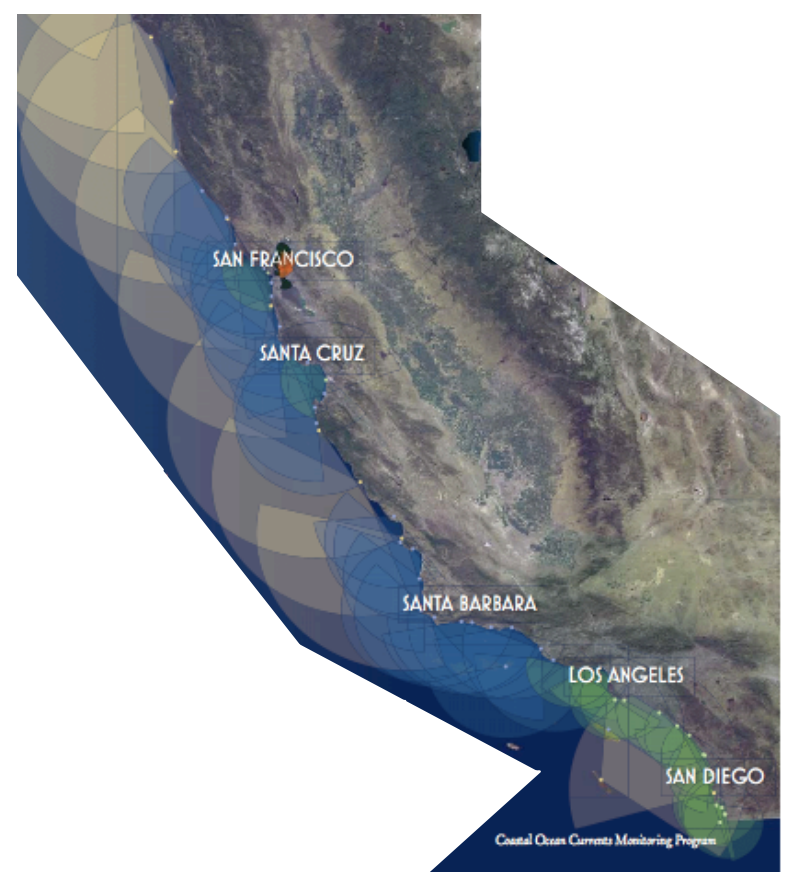

Table 2.1: The 3 color zones refer to the frequency-operating radar coverage; brown: $4 \mathrm{MHz}$, blue: $12.5 \mathrm{MHz}$ and green: $25 \mathrm{Mhz}$. The table specifies their extent and spatial resolution.

\begin{tabular}{|l|c|c|c|}
\cline { 2 - 4 } \multicolumn{1}{c|}{} & $\begin{array}{c}\text { Very Long } \\
\text { range }\end{array}$ & Long range & Short range \\
\hline Frequency & $4 \mathrm{MHz}$ & $12.5 \mathrm{MHz}$ & $25 \mathrm{MHz}$ \\
\hline Resolution & $6 \mathrm{~km}$ & $2 \mathrm{~km}$ & $1 \mathrm{~km}$ \\
\hline Up to & $150 \mathrm{~km}$ & $50 \mathrm{~km}$ & $20 \mathrm{~km}$ \\
\hline
\end{tabular}

Figure 2.7: Map of the location of the HFR along the California coastline since 2009, as well as their coverage extent (Courtesy of COMCP). 


\subsubsection{CODAR compact design}

One advantage of the CODAR sensor is that it consists of a compact one-mast antenna system, easily portable and non-obtrusive in coastal areas. It operates at a single frequency near 4, 12 and $25 \mathrm{MHz}$. Because of the physics of the propagation of the radiowaves, lower frequencies travel further. The transmit frequency determines the offshore coverage extent: $150 \mathrm{~km}(50 \mathrm{~km})$ for a $4 \mathrm{MHz}(12 \mathrm{MHz})$ operating radar frequency. CODAR consists of three co-located antennas, coaxial elements, 2 crossed loop and a monopole. The unit transmits from the vertical monopole and receives from the 3 antennas. In order to separate between the receiving and transmitting signal the Seasonde uses a Frequency Modulated Interrupted Continuous Wave (FMICW). This frequency sweeping and pulsing provides the range resolution of about $3 \mathrm{~km}$ (depends on the bandwidth available for transmission). The monopole is omnidirectional, while the loops have a cosine pattern. The signals from the 3 antennas are combined to find the direction of arrival of the radar echo using the MUltiple SIgnal Classification (MUSIC) algorithm (Barrick and Lipa, 1999 [9]).

The processing varies slightly depending on the radar operating frequency. For each antenna, every T seconds a Doppler Spectrum is computed. At $12 \mathrm{MHz}$ and an integration time $\mathrm{T}$ of 256 seconds, this results in a velocity resolution of $\triangle V=\frac{\lambda_{r}}{(2 T} \sim 4.5 \mathrm{~cm} / \mathrm{s}$ ( For a $4 \mathrm{MHz}$ system $\triangle V \sim 5 \mathrm{~cm} / \mathrm{s}$, the integration time is longer). To reduce the noise level, for each antenna, three Doppler Spectrum are averaged over 10 minutes $(\sim 18$ minutes for a 4 MHz system). For each Bragg peak where a radial current is detected, the MUSIC algorithm determines the bearing angle through analysis of the 3x3 sample-averaged covariance matrix among the three receiver signals. The largest eigenvalues are associated with a signal, the lowest with noise. There could be different locations on the sea surface with the same radial velocity. With 2 eigenvalues associated with a signal, 2 bearing angles for each identical radial speed can be solved for. But each Bragg Peak (receding and advancing) contains independent information so up to 4 bearing angles can be found. MUSIC accumulates the 
bearing angle versus radial velocity every 10 minutes ( 18 minutes for a $4 \mathrm{MHz}$ system) over an hour. Finally the bearing angles are filtered over $10^{\circ}$ stepped along every $2^{\circ}$. The end product is an estimate of radial velocities every hour with an angular resolution of $2^{\circ}$ and range resolution of about $3 \mathrm{~km}$, and a velocity resolution of $\sim 5 \mathrm{~cm} / \mathrm{s}$. (Barrick 1997 [8], Codar Ocean Sensors website http://www.codar.com)

\subsubsection{Maps of Sea surface vector currents}

Each radar station can only determine the radial component of the surface current directed toward or away from the radar. Two or more stations separated in space (typically 20-30 km apart) are needed to estimate the total two-dimensional sea surface current vector

field, by combining their radial measurements. The precision of the current velocity will vary in space because it depends on the angle between the radar radial beams pointing to the same ocean patch. For instance two radials nearly orthogonal will provide accurate current velocities. But in the baseline region, along the line of sight of two radars, where the two beams are almost parallel, it will not be possible to estimate the vector currents from the resulting near parallel radial velocities. This factor is referred to as the Geometric Dilution of Precision (GDOP), or the baseline problem. The closest to the coast the HFR can measure reliable currents depends on its frequency, and is about $9 \mathrm{~km}(4 \mathrm{~km})$ for a $6 \mathrm{~km}(2 \mathrm{~km})$ resolution product Radar signals operating at the same frequency can interfere. Since HF Radar produce data continuously, and the frequency spectrum is becoming crowded, each HF radar signal is synchronized and time-tagged with a GPS mark avoiding mutual interference.

\subsubsection{Depth extent of HFR surface currents}

The depth extent of the HFR surface current measurement is not related to the penetration depth of the emitted electromagnetic radiation, which is less then $10 \mathrm{~cm}$. Instead it depends on the extent of the waves responsible for the Bragg scattering. According to 


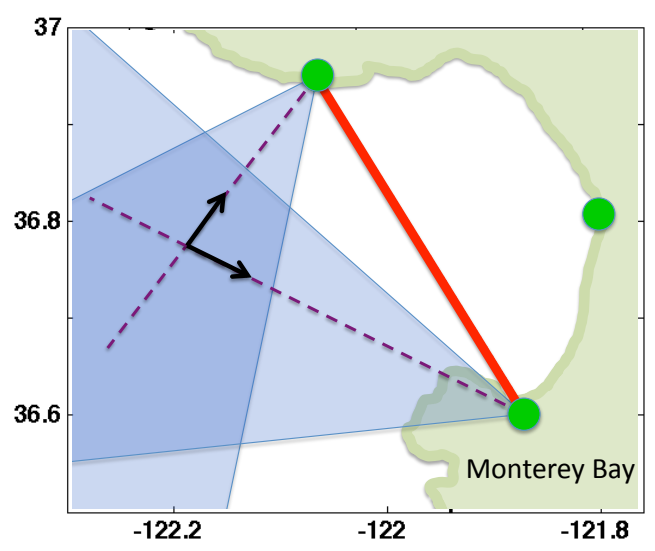

Figure 2.8: HFR geometry in Monterey Bay. Green dot: HFR radar sites. Red line: Baseline between two stations. Blue zone: part of HFR coverage area. Dotted line: radial line. Vector: radial surface current

linear wave theory the value $U_{r}$ of the current estimated from the radar (after removal of the gravity wave phase velocity) in deep water is:

$$
U_{r}=2 k_{B} \mathbf{e}_{\theta} \int_{-\infty}^{0} V(z) \exp \left(2 k_{B} z\right) d z
$$

where $\mathbf{e}_{\theta}$ is the unit vector in the radial direction toward the radar, $k_{B}$ is the wavenumber of the Bragg waves and $V(z)$ is the vertical current profile over depth z. $U_{r}$ is a depth averaged current of $V(z)$ weighted by the Bragg wave Stokes Drift profile ( Kirby and Chen 1989 [48]). Half of the weight $\exp \left(2 k_{B} z\right)$ comes from water depths less than $2.4 \mathrm{~m}$ at 4 $\mathrm{MHz}$ and $0.68 \mathrm{~m}$ at $12 \mathrm{Mhz}$. The contribution of the deeper layers decreases exponentially with depth. Therefore $U_{r}$ can be interpreted as representative of the current on the top one or two meter on the ocean. This near-surface current contains wind-driven (which includes Ekman and inertial currents) and wave-induced currents.

\subsubsection{Stokes Drift or wave-induced currents}

The phase speed of the Bragg gravity waves is computed from linear theory. But there exits a non-linear wave interaction that creates an additional current called Stokes Drift. It is the result of a single wave train interacting with itself. The deep-water waves cause 
water particles to move in a nearly circular orbit. So the trace left by the path of the water particles is not closed but progresses and results in a net transport of water called Stokes Drift (Stokes 1947 [32]) in the direction of the wave propagation. Stokes Drift is included in the current estimates because the temporal and spatial averaging of the HFR data are large relative to the wavelengths (tens of meters) and periods (a few seconds) of the Bragg waves. The Stokes Drift at the surface is typically $1 \%$ of the wind speed, much less than the Bragg wave phase speed, and decrease exponentially with depth as $\exp \left(-2 k_{B} z\right)$ (Ardhuin $2009[3])$.

\subsubsection{Measurements errors}

The precision of the radials is limited by the frequency resolution of the Doppler spectrum and is typically $2-5 \mathrm{~cm} / \mathrm{s}$. The accuracy is controlled by many factors including the Signal to Noise Ratio (SNR), pointing errors, geometry, sea-state, and the eventual presence of radio frequency interference. The distortions in the antenna beam pattern are mitigated by frequent calibration. The errors have been found to depend on the structure of the currents present (Laws et al. 2011, [49]) and are also likely to depend on the wave field. In the limit of very large SWH (SWH >5.4 m for $12.5 \mathrm{MHz}$ system) it could be impossible to make accurate measurements (Lipa and Barrick 1986 [57]). Occasionally there could be a lack of Bragg scattering in the presence of low winds. The errors can therefore be assumed to vary as a function of time with the changing currents and other conditions (Fig. 2.9). Law and Paduan (2011 [49]) review the errors in HF radar measurements from various authors. Through comparisons with current meter and drifters errors have been estimated from about 7 to $19 \mathrm{~cm} / \mathrm{s}$. Such comparisons have limitations, in part because the radar and the in-situ instrument are responsive to different horizontal scales, and do not record the current at the same depth. Using averaged values in space and time over a dense array of drifters to better simulate the HF radar measurements, lower errors of 3 to $7 \mathrm{~cm} / \mathrm{s}$ were found. Using radial velocities of multiple HF radars, errors of 5 to $12 \mathrm{~cm} / \mathrm{s}$ were reported. 


\subsubsection{HFR dataset}

In this research the hourly maps of HFR surface currents off the California coast were kindly provided by the Scripps Institution of Oceanography. Theses maps were generated with an Optimal Interpolation (OI) method from the HFR radial velocity measurements (Kim et al. 2008 [46], Kim 2010 [45]). This OI assumes a smooth spatial covariance relationship between neighboring vector currents, in contrast to the more commonly used un-weighted least square fitting method, which assumes a constant velocity within a search radius. It increases the coverage area, resolves the baseline inconsistencies and provides the mapping error uncertainties. This work analyzes the gridded optimally interpolated HFRderived surface currents off California coast for three years (2008-2010) with the resolution of $2 \mathrm{~km}$ and $6 \mathrm{~km}$ in space and hourly in time. The $2 \mathrm{~km}(6 \mathrm{~km})$ resolution HFR data have a minimum near-shore range of $3 \mathrm{~km}(9 \mathrm{~km})$ and maximum offshore range of $50 \mathrm{~km}(150$ km) (Fig. 1.1)
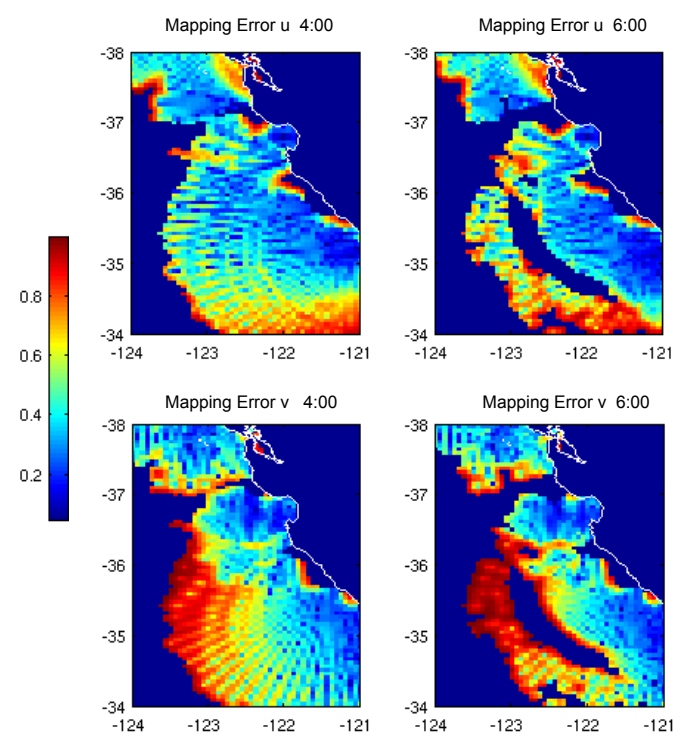

Figure 2.9: Mapping Error uncertainties provided with the gridded 6-km HFR surface currents, for (top) the zonal $(\mathrm{u})$ and (bottom) meridional (v) component, on May 23, 2009, at two different times (Left) UTC 4:00 and (Right) UTC 6:00. 
The Fig. 2.9 presents an example of the mapping error uncertainties in both the zonal (u) and meridional (v) current component which are provided with the HFR dataset. This figure shows that the uncertainties vary from time to time, but also that there are residual errors from the radials, i.e. from the location of the ocean patch relative to the radar. In this study only the current vectors with a mapping uncertainty below 0.8 in both the zonal and meridional components are kept. 


\section{Chapter 3}

\section{Dynamics in the California Current System}

\subsection{Introduction}

The California Current System (CCS) is one of the best-sampled ocean regions, yet some physical and biological processes are misunderstood and inadequately sampled, especially in the coastal zones. Quite a few studies have used satellite altimetry alone or in combination with other satellite, in-situ or model fields to investigate the CCS. They have concentrated on the variability of the features found in the several hundred kilometers off-

shore (Strub et al. 1997 [69], Kelly et al. 1998 [44], Strub and James 2002 [70], Marchesiello et al. 2003 [59]). Only recently have there been attempts to retrieve and use altimetry SSH in the coastal ocean (Saraceno et al. 2008 [67], Lee et al. 2010 [53]). The following presents the mesoscales dynamics of the CCS and checks that its variability can be captured by both the altimetry and the HFR datasets in the region of their mutual coverage.

\subsection{California Current System}

The CCS is relatively well understood at the meso and large scales and its circulation is summarized in reviews by Hickey [39] and Checkley et al. [18]. The CCS comprises two surface large-scale currents, the California Current, a broad slow surface current flowing equatorward extending about $1000 \mathrm{~km}$ offshore with a mean speed of $10 \mathrm{~cm} / \mathrm{s}$, and the coastal Davidson Current with seasonal reversals.

The CCS is an equatorward, eastern boundary current that supports one of the worlds 
largest productive coastal upwelling regions. During Spring-Summer (April to August) surface winds are aligned nearly along-shore, persistently blowing equatorward so that winddriven upwelling occurs (due to offshore Ekman transport). As a consequence, near the coast, cold nutrient rich water is upwelled from depth and is a source of biogenic surface slicks. The presence of cold water next to the coast creates a density gradient across the shelf since it is denser (lower sea levels) then warm water found offshore, and creates a strong southward flow that can reach speeds of $50 \mathrm{~cm} / \mathrm{s}$. But this current can shift when the direction and/or speed of the wind changes. The winds vary on a 2-10 day time scale, so that periods of strong southward, upwelling favorable, winds are separated by low winds (relaxation) or even northward winds, during which the upwelling jet can weaken and change its location.

In fall and winter there is continued weakening of the alongshore equatoward wind, eventually allowing for the development of the nearshore $(\sim 30-50 \mathrm{~km}$ to the coast) northward Davidson Current that can reach speeds of $1 \mathrm{~m} / \mathrm{s}$. The Davidson Current initially turns north at Point Conception and moves along the whole California coastline, then slowly reduces its span to only the Northern regions. This period is characterized by a gradual increase in sea level along the coast, and contrast with the Spring transition which happens in a periods of several days with sea levels dropping by $10 \mathrm{~cm}$ (Barth and Hickey 2005 [10]).

The majority of the seasonal variations in the CCS occurs in a relatively narrow band ( $\sim 120 \mathrm{~km}$ ) next to the coast, which is the study region of this research, and has been described using satellite altimetry data (Strub and James, 2002 [70]). This leads to the concept of a coastal transition zone (CTZ), a region of energetic jets and eddies marking the transition between the inshore coastal zone and open ocean waters.

Strong wind forcing, the coastal and bottom topography, and a narrow shelf about $10 \mathrm{~km}$ wide that transition abruptly to deep basins affect the dynamics and scales of the CCS. Coastal upwelling along coastlines with large promontories and over strongly sloping bottom topography, a source of baraoclinic instability, generates a rich eddy field. Maps 


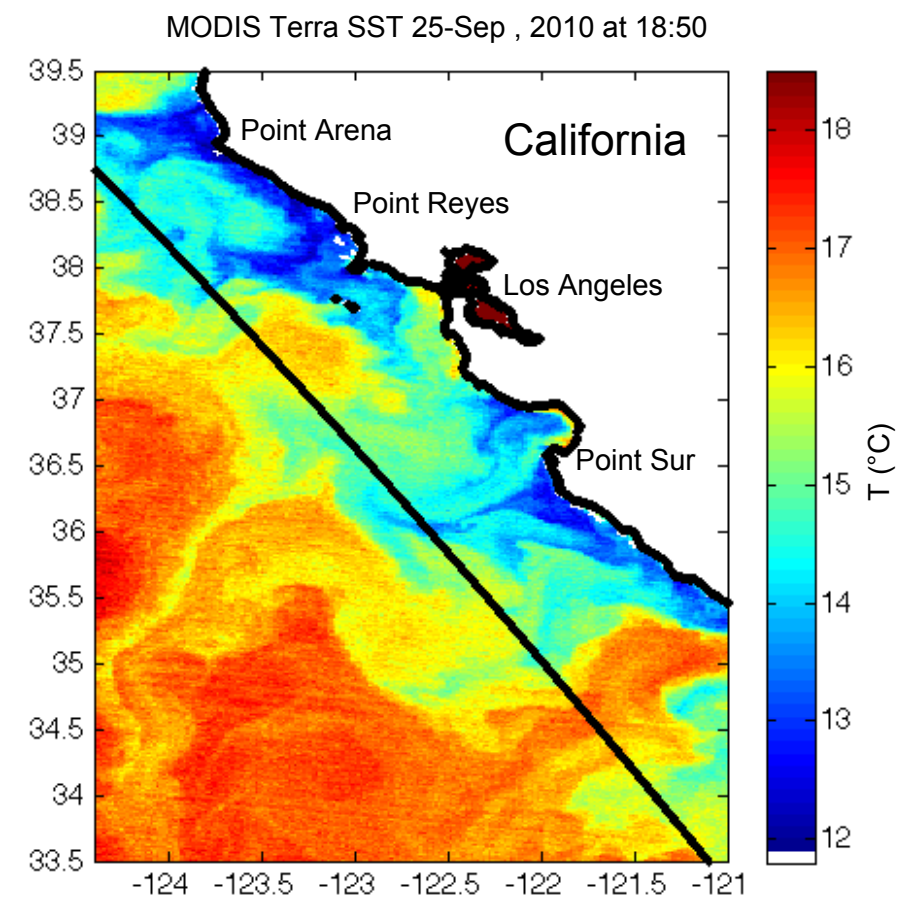

Figure 3.1: MODIS Terra SST image on Sep-25 2010, at UTC 18:50. It shows the narrow band of dark blue upwelled water next to the coast, stirred and distorted by all kinds of mesoscale eddies end jets. The black line is the Jason-1 interleaved track P206

of surface currents and SST reveal narrow energetic squirts and jets and larger-scale eddies near particular topographic features, which transport materials far from the coast. During the upwelling season the eddies are energized. Upwelling centers are typically located south of coastal headlands, where wind-stress curl is enhanced by orographic effects and where the alongshore coastal upwelling jet may turns offshore with $50 \mathrm{~cm} / \mathrm{s}$ peak velocities. The coastal upwelling jet meanders and produces narrow cold filaments extending several hundred kilometers seaward. This results in the presence of submesoscale fronts and filaments squeezed between the mesoscale and submesoscale eddies in this region (Capet et al. 2008 [16]). The upwelling cold filaments can range from submesoscale $(1-10 \mathrm{~km})$ to mesoscale $(20-100 \mathrm{~km})$ and sometimes grow as large as $200 \mathrm{~km}$. These filaments develop and decay over a period of a few weeks, and can be about $50 \mathrm{~km}$ wide at the peak of their development. The strong 
annual cycle of wind stress curl produces alongshore seasonal reversing of SSH, which then move westward.

Throughout the rest of this section and in the next chapter, the analyses focus on the variations of the large mesoscale dynamics of the CCS (100 km and 7 days). The reason is to determine if the HFR and altimeter datasets can provide the same information at these scales before investigating their relationship at the finer along track scales provided by the altimeter. The other reason is that it enables to take advantage of the weekly gridded altimetry geostrophic current maps.

\subsection{Spatial and temporal characteristics of the Ocean currents and Wind}

This section describes the mesoscales characteristics of the various components of the ocean currents: the geostrophic currents as seen by altimetry, the total surface current defined by the HFR currents, the tides and the regional wind fields which produce, among other oceanic responses, the Ekman surface drift included in the HFR currents. The spatial and temporal statistics of the products are presented. Finally a first correspondence between the altimeter and HFR current anomalies is displayed. This step is essential to verify that the geostrophic currents are dynamic and that the HFR currents can capture this geostrophic flow. Within the scope of this study the surface drifts arising from the wind-driven surface forcing, tides or other ageostrophic components are considered as noise, that need to be removed in order to extract the geostrophic signal from the HFR currents.

\subsubsection{Datasets}

We use the altimeter absolute geostrophic velocities provided in the weekly Maps of Absolute Dynamic Topography (MADT) product distributed by AVISO [6]. MADT is a gridded product on a $1 / 3^{\circ} \times 1 / 3^{\circ}$ Mercator grid. This map combines data from the entire altimeter missions. At the latitudes of California, all the along track SLA are smoothed over $100 \mathrm{~km}$ and sub-sampled every $21 \mathrm{~km}$, then the mapping uses and optimal interpolation with 
correlation space scale of $100 \mathrm{~km}$ and time scale of 10 days, to estimate the weekly gridded SLA (or MSLA). The mean dynamic topography is then added to the MSLA to obtain the Map of Absolute dynamic topography (MADT), which represents the sea surface height above the geoid. Through geostrophy the component of the geostrophic surface velocities are estimated using finite differences (Le Traon et al. 1998 [51]). This data set usually has no valid velocity values for offshore distances closer than about $30 \mathrm{~km}$, where the along track data has been flagged as bad due to its proximity to land.

The HFR currents and Winds are averaged weekly and interpolated on the same Mercator grid. In the following discussion the region of interest is concerned mainly with the valid grid points from both the HFR and altimetry currents.

The winds are from the NCEP reanalysis data provided by the NOAA/OAR/ESRL PSD, Boulder, Colorado, USA, from their web site at http://www.esrl.noaa.gov/psd/. We use the daily NCEP North American Regional Reanalysis (NARR) on a grid resolution of about $25 \mathrm{~km}$ at the Californian latitudes.

The tides are computed using the Oregon State University (OSU) Tidal Inversion Software and the OSU tidal model from the Pacific Ocean at a resolution of $1 / 12^{\circ}$ (Egbert et al. 1998, $2002[29,30])$.

\subsubsection{Ocean Currents: Mean and principal axes of variance}

The 3-year mean is observed over the region extending from $34^{\circ} \mathrm{N}$ to $39^{\circ} \mathrm{N}$. Except for the region above latitude $38.5^{\circ}$ where each product captures a strong southwestward flow, with velocities of $12 \mathrm{~cm} / \mathrm{s}(17 \mathrm{~cm} / \mathrm{s})$ for altimetry $(\mathrm{HFR})$, probably generated by the coastal promontory of Cape Mendocino $\left(40^{\circ}\right)$, the mean currents derived from HFR and altimetry (Fig. 3.2) share have few features in common. The altimetry mean field captures some eddy like patterns and a more energetic general southeastward flow $(5 \mathrm{~cm} / \mathrm{s})$ in the southern region. On the other hand, the HFR mean field presents essentially an offshore flow, with little variations in speed $(7-10 \mathrm{~cm} / \mathrm{s})$ or direction. It is expected that the two 
currents should look different, because the altimetry depicts only the geostrophic part of the currents and the optimal interpolation scales to create this product are large. On the figures there is a factor two magnification difference between the magnitude of the two mean flows.

To examine the patterns of variability of the currents the principal axes, or variance ellipses are calculated. In contrast to the mean flows, the variance ellipses share some common patterns. The magnitude and direction of the offshore variability is very similar for HFR and the altimeter currents until about the mid section of the coverage, with and a standard deviation $(\mathrm{STD})$ around $7 \mathrm{~cm} / \mathrm{s}$ in $\mathrm{u}$ and $\mathrm{v}$. Note that there is again a factor two magnification difference between the two figures. Yet the same high-energy areas can be seen. Close to the coast, the major axes of variance are alongshore, which is consistent with the fact that the low frequency parts of the coastal currents are generally steered to the coastline or bottom contours and may also include the signature of the Davidson current. The HFR currents can have a major axis STD larger than $10 \mathrm{~cm} / \mathrm{s}$, but the altimetry data is less energetic near shore compared to the HFR currents and this may be an artifact of the altimeter gridded product generated using large covariance scales.

The region above $38^{\circ} \mathrm{N}$, presents the largest variability with an STD of $10 \mathrm{~cm} / \mathrm{s}(15$ $\mathrm{cm} / \mathrm{s}$ ) for the altimeter (HFR) currents. Offshore the major axes of the HFR are generally oriented cross-shore. The magnitudes of the major axes for the altimetry currents are similar to the minor axes, indicating a relatively turbulent eddy-like flow. The HFR variability depicts a less turbulent flow with larger major axes magnitudes than the minor axes.

The tidal variability (not shown) is almost 10 times less than the HFR variability, in the exception of the near shore. The tides may reach $30 \mathrm{~cm} / \mathrm{s}$ during the high tide season (at the 10-km resolution grid), but their amplitude is usually less than $10 \mathrm{~cm} / \mathrm{s}$. The tides represent one component of the current, but are not dominant. Consequently, it will not overwhelm the geostrophic part of the current contained in the HFR signal. Eventually more detailed analyses would be required near shore as the tides depend on local bottom topography and coastline geometry. 

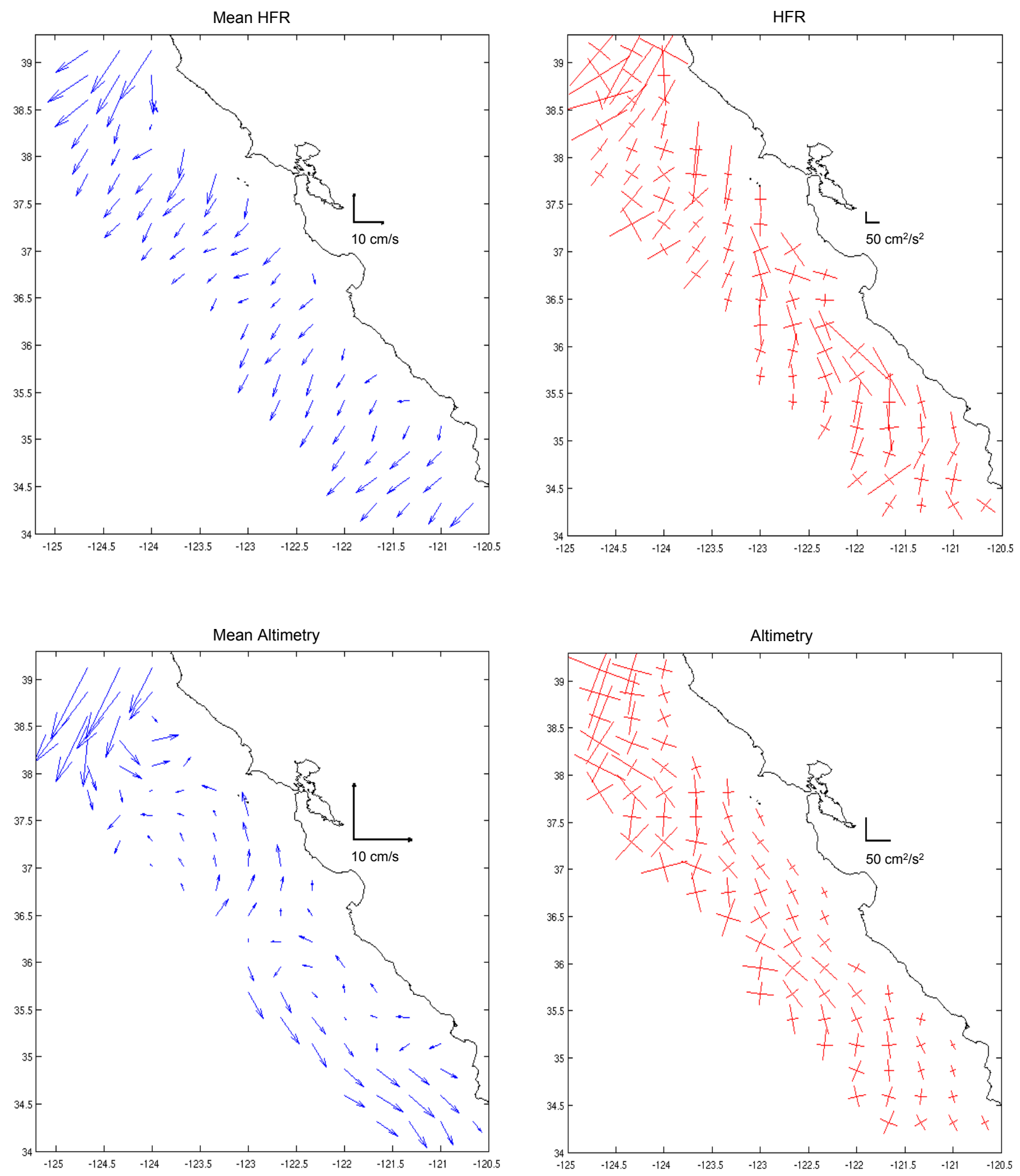

Figure 3.2: 3-year mean (Right) and Principal Axis of Variance (Left) for HFR currents (top), Altimetry currents (middle), in the region where altimetry and HFR currents are coincident on a $25-\mathrm{km}$ resolution grid 


\subsubsection{Wind: Mean and Principal axes of variance}

Seasonal wind variations are large and play an important role in the ocean dynamics. The winds vary on a 2-10 day time scale. The California coastal region usually experiences its strongest winds (mean 8-10 m/s) during the spring months (March- May). Light winds $(5 \mathrm{~m} / \mathrm{s})$ are associated with the autumn months (August- October). The 3-year mean wind velocity of $5 \mathrm{~m} / \mathrm{s}$ is oriented primarily alongshore in the southeastward direction (Fig. 3.3) as well as its major axes of variance. The magnitude of the major axes increases to the north, and its related STD almost doubles from $3 \mathrm{~m} / \mathrm{s}$ at $34^{\circ} \mathrm{N}$ to $6 \mathrm{~m} / \mathrm{s}$ at $39^{\circ} \mathrm{N}$. During spring and summer, wind direction is primarily to the south. In fall and winter the poleward wind stress increases, eventually reversing in the winter north of $38^{\circ} \mathrm{N}$. The wind-generated surface Ekman current varies proportionally to the wind speed squared, and is about 2.5 $\mathrm{cm} / \mathrm{s}(10 \mathrm{~cm} / \mathrm{s}$ and $20 \mathrm{~cm} / \mathrm{s}$ ) for the mean $5 \mathrm{~m} / \mathrm{s}$ wind speed (for a $10 \mathrm{~m} / \mathrm{s}$ and a $15 \mathrm{~m} / \mathrm{s}$ wind speed). It is present in the HFR current.
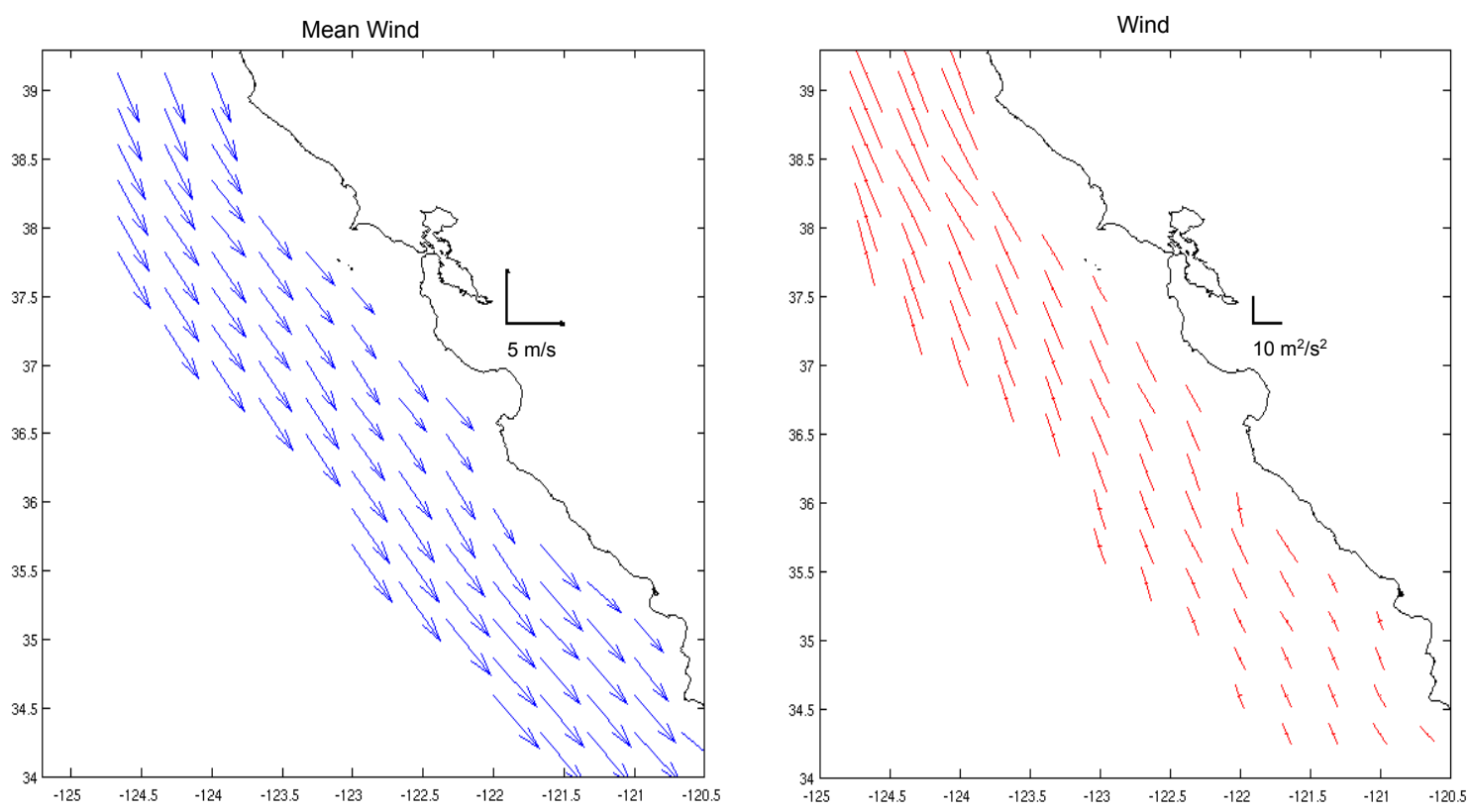

Figure 3.3: 3-year mean (left) and Principal Axis of Variance (right) for the Winds, in the region where altimetry and HFR currents are coincident on a $25-\mathrm{km}$ resolution grid 


\subsubsection{Ocean Currents and Wind time series}

This section presents the 3-year time series of zonal and meridional ocean currents and winds, averaged over the complete $100-\mathrm{km}$ wide coastal strip between latitude $34^{\circ}$ and $38^{\circ} \mathrm{N}$. This area contains 54 grid points. The weekly gridded HFR wind and altimetry datasets are each processed in a slightly different way in time and space. It is noticed that the 3-year time series of the HFR and wind observations are noisier than the one obtained from the altimetry data ((Fig. 3.4 and Fig. 3.5). An additional 5-week moving average is chosen to be applied to the HFR and wind 3-year time series to clarify the patterns included in the time series and to match the smoothness of the altimetry time series.

The HFR and altimetry ocean current time series (Fig. 3.4) show that the two currents behave differently. The currents oscillate around distinct means, and the HFR level of variability is much larger. For instance the altimetry has a positive zonal current (eastward) around $3 \mathrm{~cm} / \mathrm{s}$, and a negative (southward) meridional current around $-3 \mathrm{~cm} / \mathrm{s}$. On the contrary, the more energetic zonal HFR currents are almost always negative (westward) and the meridional currents oscillate between positive (northward) and negative (southward) currents.
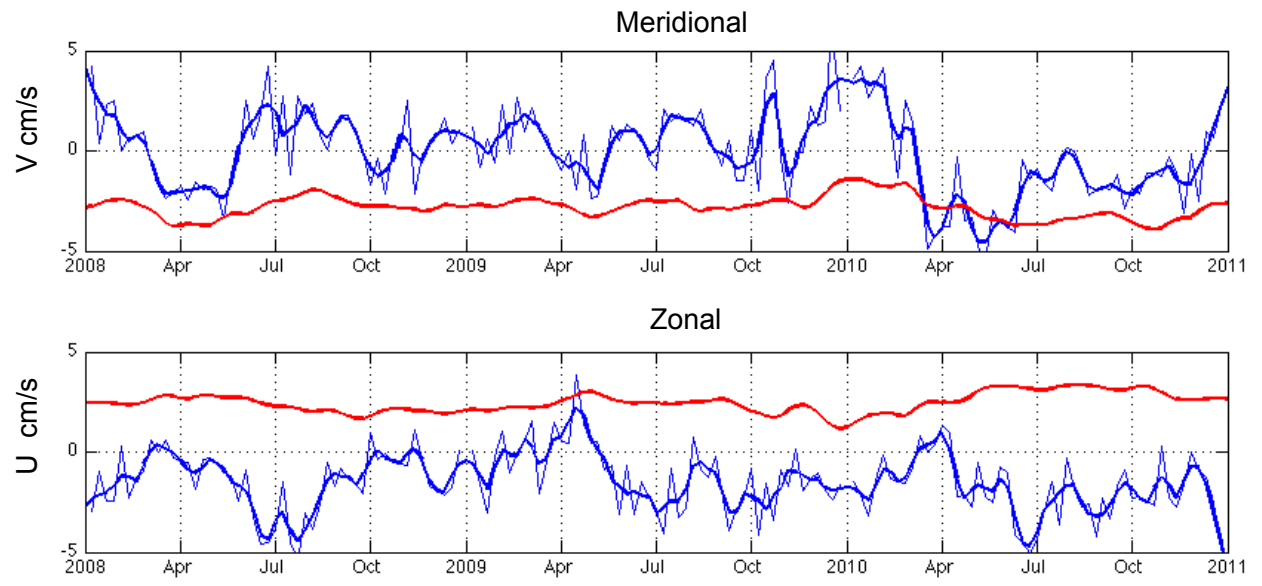

Figure 3.4: Meridional and zonal 3-year time series averaged over the 100-km wide coastal strip for HFR (in blue) and Altimetry (in red) currents 
However, the time series of the current anomalies (Fig. 3.5 top) provide a more satisfying comparison. The meridional anomalies show a strong annual periodic behavior with of northward flow in spring. The zonal anomalies pattern is less clear, although there is a systematic westward flow in fall and winter. The two meridional current anomalies are very similar with a correlation of 0.7 . The zonal current anomalies are also consistent but with more offsets, especially at the beginning of each year, and have a correlation of 0.5. These offsets seem to correspond with strong wind anomalies, relative to a mean wind of $5 \mathrm{~m} / \mathrm{s}$ flowing southeastward (Fig3.5 middle): a higher meridional velocity tending to be positive and a lower (almost null) zonal velocity component (i.e. the winds are low or flowing northward), which also match the high peaks in the wind Eddy Kinetic Energy (EKE) (Fig. 3.5 bottom).

The EKE represents the variable part of the horizontal kinetic energy per unit mass at a point contained within the flow of a fluid, i.e. after removing the Mean Kinetic energy associated with the mean velocity. The term "Eddy" flow in this context refers to the current anomaly.

$$
E K E=\frac{1}{2} \overline{(u-\bar{u})^{2}+(v-\bar{v})^{2}}
$$

where $\mathrm{u}$ is the zonal current component and $\bar{u}$ its time mean, and $\mathrm{v}$ is the meridional current component and $\bar{v}$ its time mean. The time series of the EKE presents another perspective to check the dynamics of the currents, their similarities and inconsistencies.

The altimetry and HFR EKE oscillations match well, with the HFR EKE having larger amplitudes because it contains, a part connected to the wind forcing variability. The large peaks in the HFR EKE (April-June) seem to lag after the large peaks in the wind EKE (February). Only 3-years of observation does not allow to clearly define the ocean annual oscillation structures especially because there is an El Niño starting fall of 2009 and persisting until spring of 2010. The typical wind patterns are altered and can change the ocean dynamics. In California, the northwest winds tend to be weaker during El Niño events, 

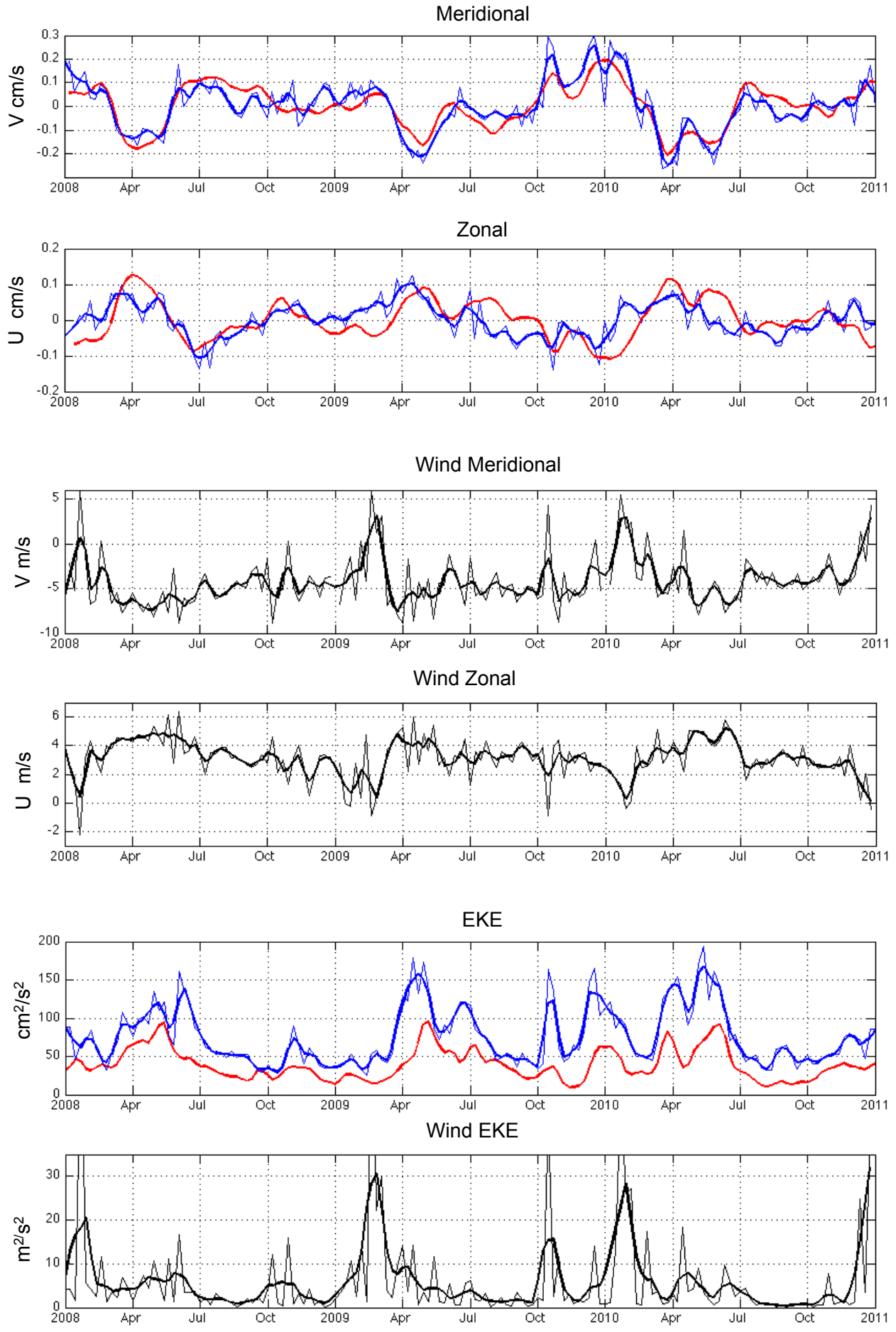

Figure 3.5: Meridional and zonal 3-year time series averaged over the 100-km wide coastal strip (top) for HFR (in blue) and Altimetry (in red) currents anomalies; (middle) for the winds (in black). (bottom) 3-year time series of the EKE for the HFR (in blue) and altimetry (in red) currents and for the winds (in black) 
which reduces the coastal upwelling.

In summary, this chapter provides the large scales characteristics and dynamics that prevail in the California Coastal Current. The wind forcing plays a large role in the ocean dynamics, and its upwelling pattern. The tides are not dominant. The altimetry and HFR current anomalies are comparable, and they are dynamic with significant temporal variations. So the aim of this research study, which is to define a methodology to retrieve the geostrophic component from the HFR makes sense and can be undertaken. And because the HFR signal contains the wind induced surface drift, we expect this relationship to depend on the wind patterns. In the following chapter, the study will continue using the gridded altimetric product to experiment with a technique to extract the large-scale geostrophic HFR currents. In subsequent chapters the methodology will be refined to take into account the smaller mesoscale features that occur along the California coast and will be investigated using the along track altimeter data. 


\section{Chapter 4}

\section{Processing of the HFR currents for the gridded Altimetry product}

\subsection{Introduction}

This chapter defines the methodology to process the HFR currents in order to make them comparable with the gridded altimetry observation at the large mesoscales $(100 \mathrm{~km}$ and 7 days). First the technique will be developed for the ocean currents, then an SSH product from the HFR currents will be generated and analyzed with respect to the altimeter SSH measurements.

\subsection{Estimating geostrophic currents from the HFR surface currents}

\subsubsection{Ekman Correction}

The strong offshore flow seen in the HFR mean field (Fig. 3.2) demonstrates little eddy variability, which is the characteristics of a geostrophic flow. This indicates that the HFR currents measure also a surface ageostrophic current. The residuals of the mean HFR currents minus the mean geostrophic currents derived from satellite altimeter gives an estimate of the mean horizontal ageostrophic currents. The residuals show velocities that are predominantly to the right of the wind and show a coherent spatial structure (Fig.4.1). This suggests that the residuals contain the Ekman surface drift induced by the effect of wind stress on the sea surface and the Earth's rotation. Ekman (1905 [31]) first theorized this effect on the upper ocean and concludes that in the northern hemisphere, the drift current at the 
surface will be deviated $45^{\circ}$ to the right of the wind, relative to the water. To simplify the mathematical treatment of the problem, Ekman assumed a steady wind, a large ocean of uniform depth, water as an incompressible fluid and a constant coefficient of turbulent viscosity. The influence of neighboring ocean currents and continents are neglected. This leads to a solution at depth z below the surface:

$$
V_{E}=V_{0} \exp \left(-\pi \frac{z}{D_{E}}\right) \exp \left(-i\left(\frac{\pi}{4}+\pi \frac{z}{D_{E}}\right)\right)
$$

In these expressions, the notation $V=u+i v$ is a complex current vector; $V_{0}$ is in the direction of the wind $W$ and its magnitude provides the velocity of the water at the sea surface:

$$
V_{0}=\frac{T}{\rho_{w} \sqrt{A_{\nu} f}}
$$

Here, $T$ is the wind stress given by the Bulk formula $\rho_{\text {air }} C_{\text {drag }}\|W\| W ; \rho_{\text {air }}$ is the density of air $\left(2.22 \mathrm{~kg} / \mathrm{m}^{3}\right) ; C_{d r a g}$ is the drag coefficient $\left(1.210^{-3}\right) ; A_{\nu}$ is the coefficient of turbulent viscosity $\left(0.01 \mathrm{~m}^{2} / \mathrm{s}\right) ; \rho_{w}$ is the density of water $\left(1025 \mathrm{~kg} / \mathrm{m}^{3}\right) ; f$ is the Coriolis parameter given by $f=2 w \sin (\phi)$ at geographical latitude $\phi$ and $w$ is the Earth's angular velocity of rotation (7.29 10-5 radian/s); $D_{E}$ is the Ekman depth or depth of frictional influence. These equations predict a spiral structure for the currents in the surface layers of the sea, with a decrease of current speed with increasing depth, and a progressive deviation on the direction of the deeper current. This wind driven Ekman spiral becomes fully developed in a day or two after the rise of the generating wind. $D_{E}$ is usually around $30 \mathrm{~m}$ but varies with location and season. The HFR current represents an average current over the top $1 \mathrm{~m}$ of the ocean surface. So the simplified expression will be used:

$$
V_{E}=\alpha_{E} V_{0} \exp \left(-i \varphi_{E}\right)
$$

derived from Eq4.1 to express the Ekman current observed by the HFR, where $\alpha_{E}$ is the amplitude relating the Ekman current to the surface winds and $\varphi_{E}$ is the angle between the wind and Ekman current, with positive values indicating a current rotated clockwise from the wind direction. In theory at the surface $\alpha_{E}=1$ and $\varphi_{E}=\pi / 4$. 


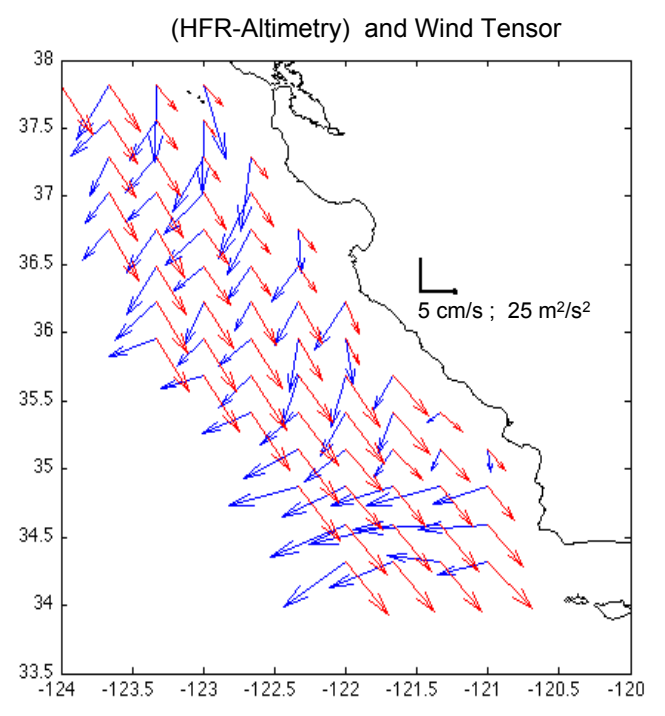

Figure 4.1: Residuals of the mean HFR currents minus mean Altimetry currents (in blue); 3-year mean wind tensor $\|W\| W$ (in red) on a $25 \mathrm{~km}$ resolution grid

The parameters $\alpha_{E}$ and $\varphi_{E}$ are estimated for each of the 54 collocated grid points, using a least square technique to find the parameter values that minimize the sum of the squares of the residuals between the modeled and the observed Ekman currents over 3 years of weekly data. Here the mathematical model is from Eq4.2 and is computed using the NCEP winds; the observations are the observed Ekman currents derived from the residuals HFR minus Altimeter currents. The observations have some errors, but these are ignored in this simple estimation technique.

Fig. 4.2 shows the spatial distribution of the parameters $\alpha_{E}$ and $\varphi_{E}$. $\varphi_{E}$ is usually more than $60^{\circ}$, with lower values in the nearshore regions, and $\alpha_{E}$ is around 1 , with higher values nearshore. Since the color scales vary in opposition, they reveal a similar spatial structure, except around $34^{\circ} \mathrm{N}$. Near the coastline, there is a periodic pattern of increasing and decreasing $\varphi_{E}$. Further offshore the distribution is more uniform, with the intrusion of filament like structure more evident for $\varphi_{E}$, around $36^{\circ} \mathrm{N}$, the other for $\alpha_{E}$ around $34^{\circ} \mathrm{N}$. The southern region reveals deeper values of $\varphi_{E}$ turning as much as $110^{\circ}$. Similar structures were also observed by Matthews (2008 [43]) with Ekman currents estimated from ocean currents 
calculated from Sea Surface Temperature (SST) satellite measurements and altimeter data.
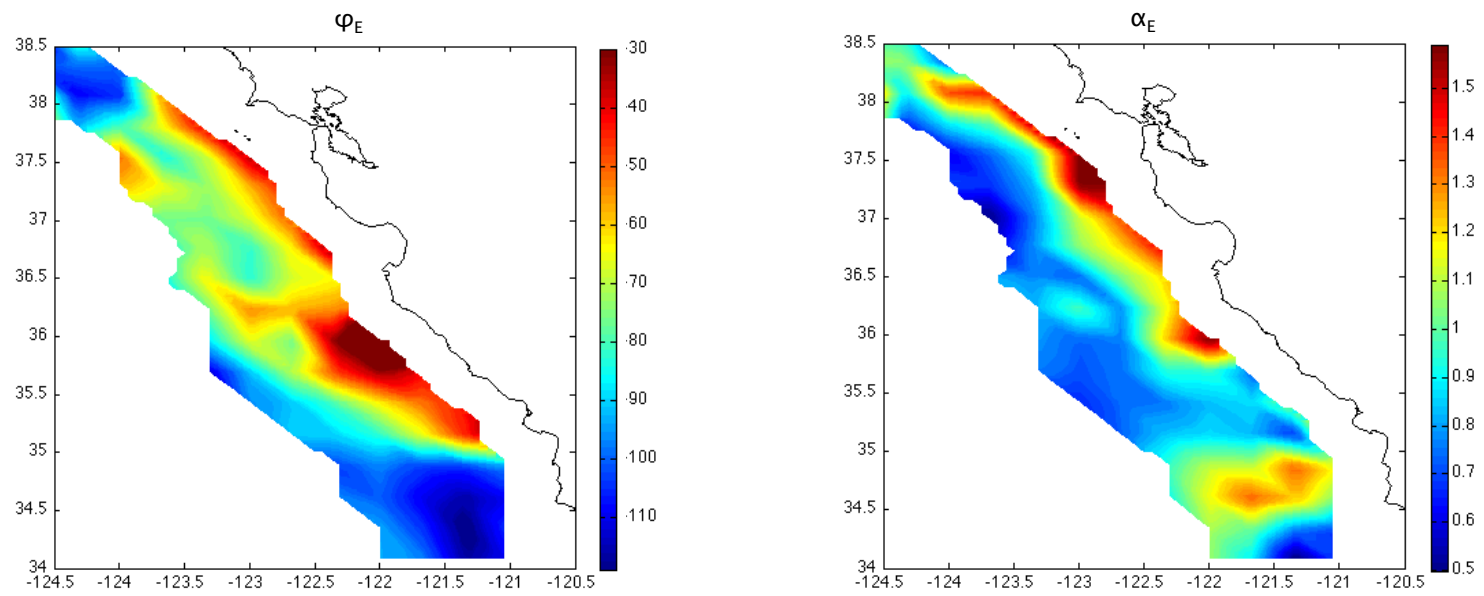

Figure 4.2: Estimates of $\varphi_{E}$ in degrees (left) and $\alpha_{E}$ (right) from the Least Squares
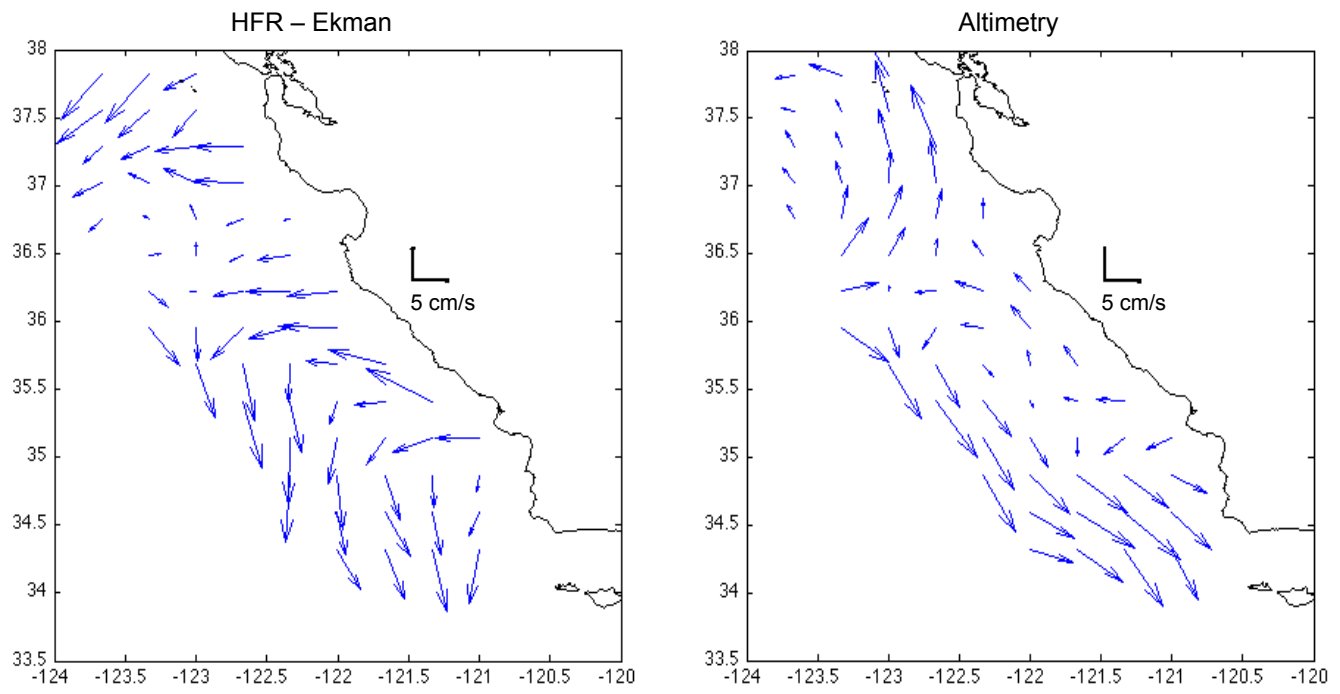

Figure 4.3: 3-year mean of the residuals HFR - Estimated Ekman currents (left) and 3-year mean Altimetry currents (right)

The relationship that exists between $\varphi_{E}$ and $\alpha_{E}$ arises from their connection via the Ekman depth as expressed in the theoretical formulation: $\varphi_{E}=-\pi / 4+\pi z / D_{E}$ and $\alpha_{E}=\exp \left(-\pi z / D_{E}\right)$. A deeper Ekman depth will induce less rotation and a larger am- 
plitude coefficient, and indicates where the frictional influence of the wind is higher. When the Ekman depth derived from the first does not correspond to the second, there is a breakdown in the classical Ekman solution. Also the Ekman depth may be limited by a vertical stratification with larger velocities in shallow mixed layers and directions of $V_{E}$ more strongly deflected to the right of the wind than previously expected. For example, Rascle (2007 [62]) extrapolated to the surface measurements from mooring data and found directions of $V_{E}$ between $45^{\circ}$ and $90^{\circ}$, more than the $45^{\circ}$ given by the constant eddy-viscosity model of Ekman. Analyses of HFR data by Ardhuin et al. (2009 [3]) give an average Ekman deviation of $60^{\circ}$.

The two spatially dependent Ekman parameters are used to generate a 3-year time series model of the Ekman currents, which are then removed from the HFR total currents. This HFR corrected Ekman current should provide a better estimate of the geostrophic current. Indeed the 3-year mean of the HFR corrected Ekman current now reveals quite similar patterns to the altimeter derived geostrophic currents (Fig. 4.3), as opposed to the persistent offshore flow from the mean HFR currents (Fig. 3.2). The RMS of the zonal and meridional components between the altimetry and the HFR Ekman corrected currents decreases from an RMS in $\mathrm{u}=5.5 \mathrm{~cm} / \mathrm{s}$ and RMS in $\mathrm{v}=5.4 \mathrm{~cm} / \mathrm{s}$ (using HFR only) to an RMS in $\mathrm{u}=1.8 \mathrm{~cm} / \mathrm{s}$ and RMS in $\mathrm{v}=2.2 \mathrm{~cm} / \mathrm{s}$, an improvement of more than $60 \%$.

The quality of the Ekman correction is important, and probably depends on the season because of the changes in the ocean stratifications, and also the wind history since the Ekman theory assumes a steady wind. But this Ekman correction is not easy to estimate because there exist other ageostrophic surface current components. The surface wind also induces a Stokes Drift, and baroclinic currents and other phenomena that are difficult to separate from the wind-driven component. The quantitative variations of surface drift as a function of forcing parameters is still relatively poorly understood. 


\subsubsection{Optimal Interpolation to force geostrophy}

The methodology is explained in detail in Roesler et al. (2013 [65]), a short version is given here.

Altimetry maps the vertically integrated $\mathrm{SSH}$ that can be related to the geostrophic flow, whereas HFR data gives us a surface total velocity and includes ageostrophic processes that need to be removed to compare the two data sets. In this research an Optimal Interpolation (OI) is chosen to retrieve the geostrophic currents from the total velocity surface measured by the HFRs. More precisely, the OI estimates the non-divergent stream function and, assuming near-geostrophy, the HFR two-dimensional SSH and geostrophic velocity fields. The OI requires an analysis of the time and spatial scales of the coastal oceanic features.

The variable to be estimated is the stream function $\psi$, because we are interested in capturing the geostrophic (non-divergent) part of the HFR observed-flow. If the flow is assumed to be nearly geostrophic, there is a linear relationship between the stream function and the observed velocities: $(u=-d \psi / d y$ and $v=d \psi / d x)$. The stream function $\psi$ can, then, be calculated directly from the HFR derived currents using an OI (Bretherton et al. 1976 [13], Wilkin et al. 2002 [75]). The OI forces the flow to be non-divergent, but if the assumptions of near geostrophy are erroneous, this non-divergent field may not be representative of the true geostrophic structures.

The mapping procedure using an Optimal Interpolation (OI) generates weekly maps of geostrophic currents from the HFR total currents, on the MADT $1 / 3^{\circ} \times 1 / 3^{\circ}$ resolution grid. The time and spatial scales of the CCS oceanic features are derived from an analysis

of the observed HFR velocity structures. In the open ocean $(\geq 50 \mathrm{~km})$, the time scales are about 7-10 days and the spatial scales larger than $60 \mathrm{~km}$, with the Walstadt covariance function fitting the observed spatial covariance function from the HFR data.

Before applying the OI, the HFR currents are averaged over a week, which is consistent 
with the time scales of the mesoscale features. This removes the effects of tides and wind generated inertial currents. The velocity noise error for the HFR is assumed to be constant $e=15 \mathrm{~cm} / \mathrm{s}$ although in reality this error varies depending primarily on the radar and current geometry. This value is chosen considering the typical errors found in the HFR velocities. In this section the OI uses the realistic Walstadt covariance function with spatial scales of $60 \mathrm{~km}$. As long as we are studying the coincident coverage area of the altimeter and HFR current fields, the OI mapping error is constant throughout because the underlying HFR are gridded with a resolution of $6 \mathrm{~km}$ and there are rarely missing data over a weekly period. The number of averaged points on a particular grid point over a week was not taken into consideration and is a supplemental uncertainty in the HFR observations.

\subsection{Comparison of Altimetry currents with various HFR processing}

The effect of using several methods to process the HFR data before comparing it with the altimetry currents anomalies are analyzed. Four options are considered:

(1) simply use the HFR as given.

(2) remove the Ekman current from the HFR.

(3) force geostrophy by applying the OI.

(4) remove the Ekman current from the HFR before applying the OI.

All these products are re-gridded to the altimeter grid

\subsubsection{3-year time series of Current anomalies averaged over the 100-km wide coastal zone}

The first option was already presented in the previous chapter in Fig. 3.5 top and displays that, already, when there is no specific processing the meridional component of HFR and altimeter current anomalies are surprisingly well correlated (0.75). The most significant 
differences appear for the zonal current anomalies, especially in the winter times; and this is the component that will be analyzed more in depth here. Although the correlation and RMS for all the options and both meridional and zonal current components are summarized in Table 5.1. Fig. 4.4 shows the 3-year time series of the zonal current anomalies averaged over the coincident 100-km wide coastal strip for different options of HFR current processing, together with the altimeter data. This is first improved by removing the Ekman currents from the HFR (Fig. 4.4 middle), the correlation for the zonal part increases from 0.45 to 0.7. This 3-year time series also reveals that the estimated Ekman parameters may not be adequate for all seasons and/or weather conditions. For example, in the fall 2010 removing the Ekman currents creates an additional offset that is not present in the original HFR.

However the third option by forcing geostrophy provides a clear improvement (not shown) with a zonal correlation of 0.83 , expect that this smoothes the OI processed currents by a factor of 1.7. Once this multiplicative factor is applied, the RMS for the zonal and meridional current anomalies are reduced by $50 \%$ compared to the first option. Finally there is only a marginal difference between the method 4 (OI and Ekman Corrected) and the previous method (OI only). For this reason only the OI and Ekman Corrected method is presented in Fig. 4.4 bottom.

To conclude, for the gridded product, at the large time and spatial scales, the OI method provides the most noticeable approximation to geostrophy, and adding the removal of the Ekman component only improves slightly the geostrophy. In the following sections the OI Ekman Corrected method will be used for further quantitative comparisons.

\subsubsection{Spatial statistics using the OI and Ekman Corrected HFR method}

The spatial distribution for the 3-year correlation between the weekly altimetry and HFR current anomalies is presented, for each 54 grid-points. Fig. 4.5 displays the correlation obtained from two HFR products: the first one derived from method 1 (HFR only) and the second one generated by the OI Ekman Corrected method (method 4). 


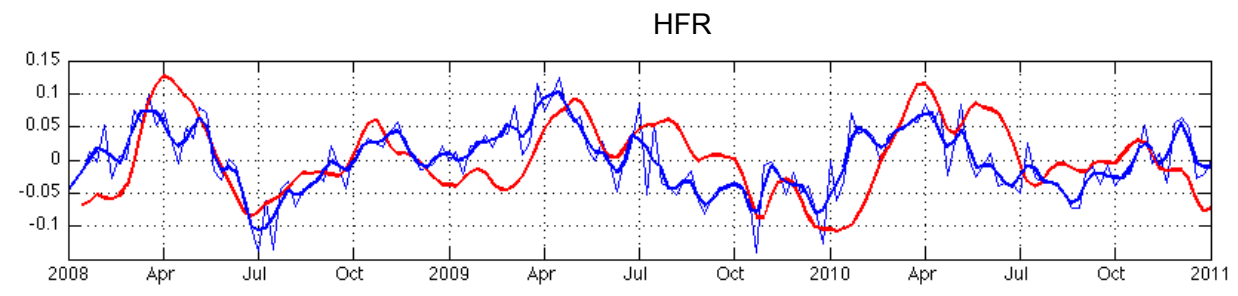

HFR Ekman Corrected
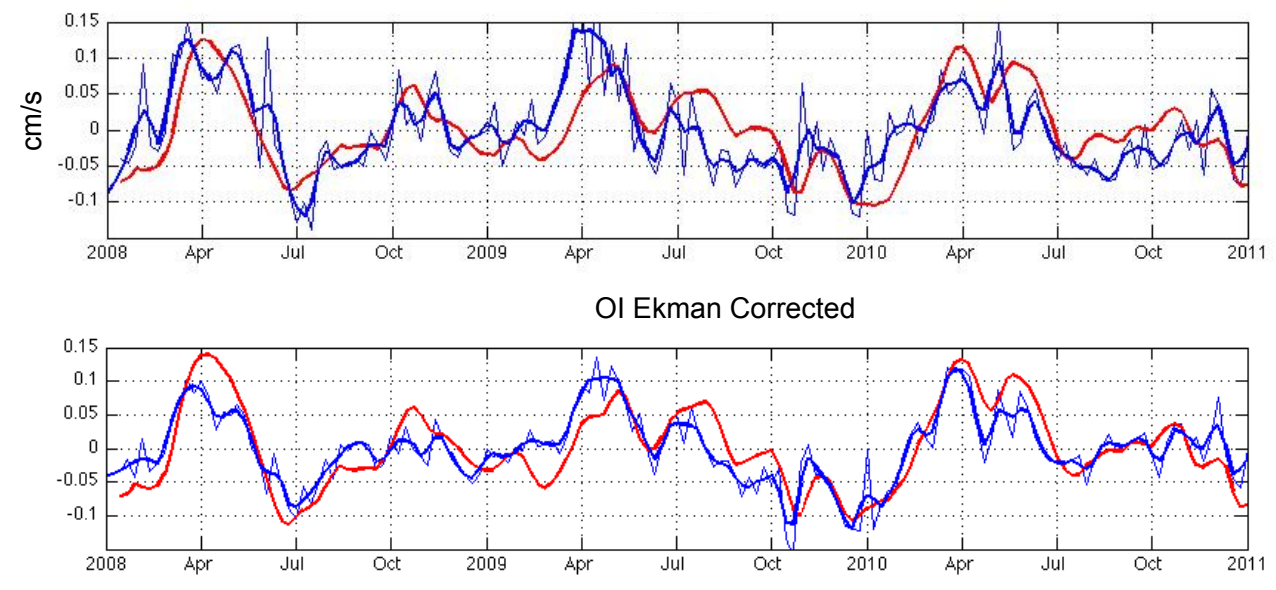

Figure 4.4: 3-year time series of Zonal current anomalies for various processing of the HFR currents. Top: HFR only, Middle: Ekman Corrected HFR, Bottom: OI Ekman Corrected HFR

Table 4.1: Statistics for the 3-year time series of zonal (u) and meridional (v) current anomalies averaged over the 100-km wide coastal zone, between altimetry and 4 processed HFR sets.

\begin{tabular}{|c|c|c|c|c|}
\cline { 2 - 5 } \multicolumn{1}{c|}{} & HFR only & $\begin{array}{c}\text { HFR Ekman } \\
\text { Corrected }\end{array}$ & OI only & $\begin{array}{c}\text { OI and Ekman } \\
\text { Corrected }\end{array}$ \\
\hline Corr u & 0.45 & 0.7 & 0.83 & 0.83 \\
\hline Corr v & 0.77 & 0.85 & 0.89 & 0.89 \\
\hline RMS u (cm/s) & 0.16 & 0.16 & 0.066 & 0.065 \\
\hline RMS v (cm/s) & 0.16 & 0.1 & 0.045 & 0.044 \\
\hline
\end{tabular}

Both methods give very similar spatial distribution of the correlation, but the correlations calculated from the OI Ekman Corrected HFR currents show more spatial uniformity and an improvement of $20 \%$ compared to the unprocessed HFR (Table 4.2). Overall the 
meridional currents agree better with correlations larger than 0.5 except in the central area of $36^{\circ} \mathrm{N}$. The low correlation areas differ for the zonal and meridional currents. The main improvements are for the zonal component with weaker correlations in two regions, the southern part and the western area around $37^{\circ} \mathrm{N}$. But even after the amelioration the correlations stay low in these regions around 0.35 .

Table 4.2: The 3-year mean correlation between altimetry and the various processed HFR current anomalies. The mean correlation for the zonal $(\mathrm{u})$ and meridional (v) current anomalies is computed for the 54 collocated grid points.

\begin{tabular}{|c|c|c|c|c|}
\cline { 2 - 5 } \multicolumn{1}{c|}{} & HFR only & $\begin{array}{c}\text { HFR Ekman } \\
\text { Corrected }\end{array}$ & OI only & $\begin{array}{c}\text { OI and Ekman } \\
\text { Corrected }\end{array}$ \\
\hline Corr( $\mathrm{u})$ & $0.46 \pm 0.23$ & $0.5 \pm 0.23$ & $0.56 \pm 0.22$ & $0.57 \pm 0.22$ \\
\hline $\operatorname{Corr}(\mathrm{v})$ & $0.53 \pm 0.16$ & $0.54 \pm 0.16$ & $0.58 \pm 0.15$ & $0.6 \pm 0.14$ \\
\hline
\end{tabular}

To get another quantitative measure of how well the altimetry and OI Ekman Corrected HFR currents agree, their complex correlation is calculated. Complex correlation is defined as follows (after Kundu, 1976[42])

$$
\rho=\frac{\overline{V_{A L T} V_{H F R}^{\star}}}{\sqrt{\overline{V_{A L T} V_{A L T}^{\star}} \overline{V_{H F R} V_{H F R}^{\star}}}}=|\rho| e^{i \phi}
$$

where the velocity field $(\mathrm{u}, \mathrm{v})$ for either altimetry (ALT) or HFR is represented by the complex number $\mathrm{V}$, and $\mathrm{V}^{\star}$ is its complex conjugate. Physically, the magnitude correlation $|\rho|$ is a measure of the relative magnitude variations between the two datasets. The phase angle $\phi$ gives the mean counter-clockwise rotation of the HFR radar current vectors with respect to the altimeter vectors, and is meaningful only when the magnitude correlation is high.

Complex correlations were computed for each of the 157 sets of coincident HFR and altimetry vectors and then averaged over 3 years. They were calculated for each option of processed HFR and the results are summarized in Table 4.3. Fig. 4.6 displays their spatial 
a)

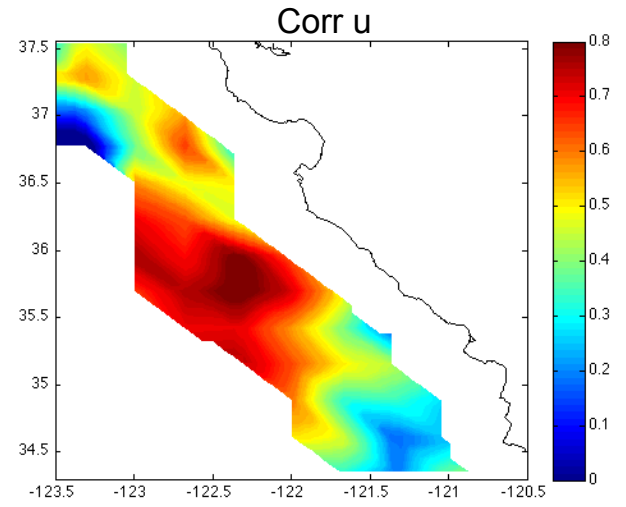

b)

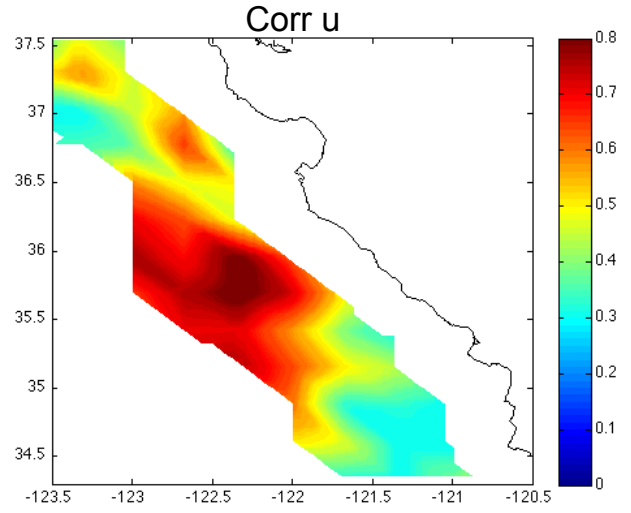

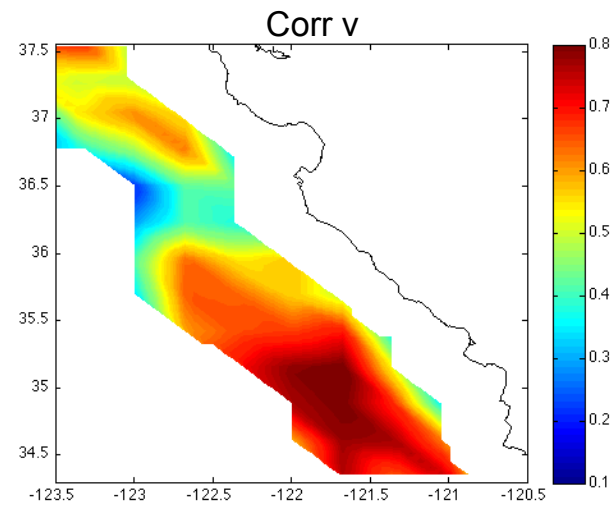

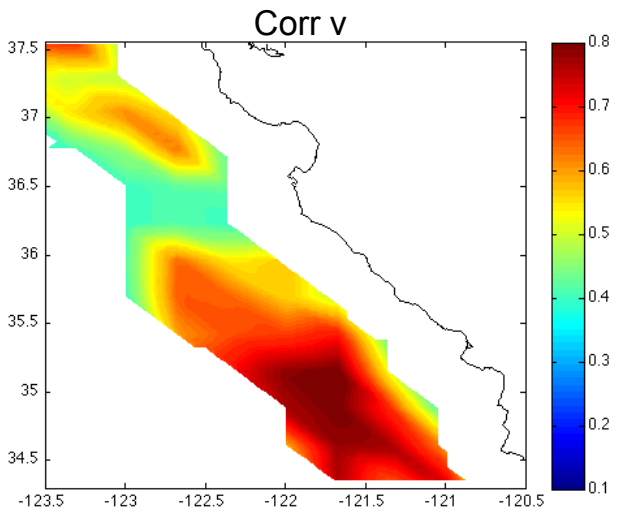

Figure 4.5: a) 3-year mean correlation in $\mathrm{u}$ and $\mathrm{v}$ between altimetry and OI Ekman Corrected HFR current anomalies. (b) 3-year mean correlation in $\mathrm{u}$ and $\mathrm{v}$ between altimetry HFR current anomalies.
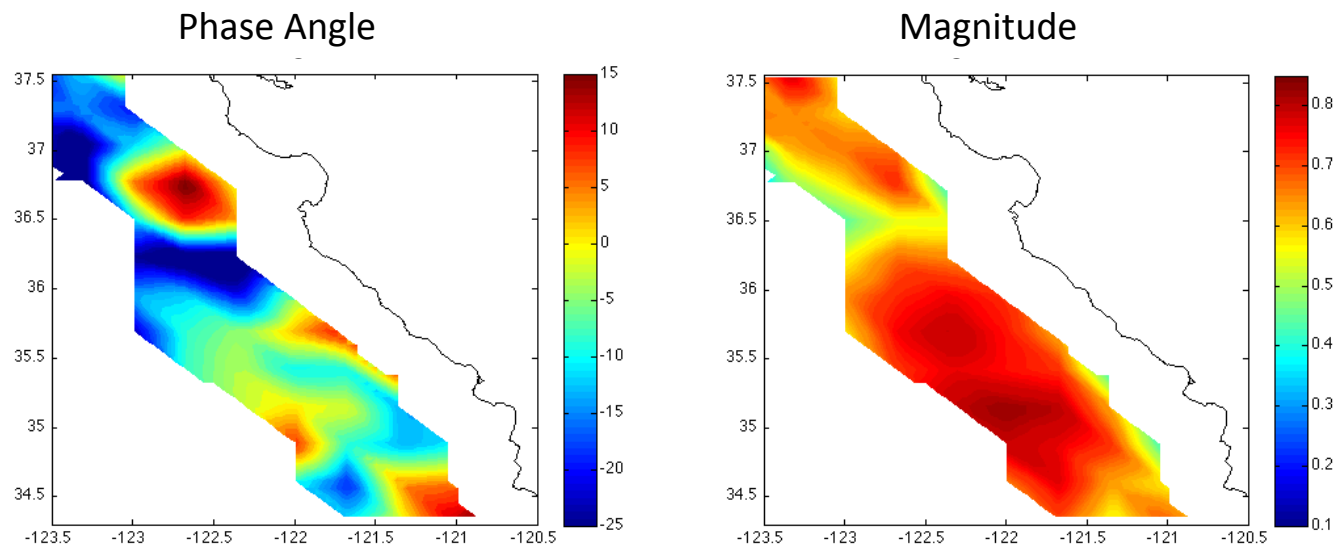

Figure 4.6: 3-year Mean Complex correlation between altimetry and OI Ekman Corrected HFR current anomalies (left) Phase Angle (right) Magnitude 
distribution for the OI Ekman Corrected method. The magnitude correlation is well uniformly spread with values mainly larger than 0.6 and a mean of 0.72 , so that the correlation angle can easily be interpreted. The spatial distribution of $\phi$ reveals that there are more differences in direction of the currents in the Northern section, varying between $-25^{\circ}$ and $15^{\circ}$, so that the direction errors in the geostrophic derived HFR currents, seem related to specific oceanic conditions. The mean correlation angle is $-2 \pm 15^{\circ}$.

Table 4.3: The phase and magnitude of 3-year mean Complex correlation between altimetry and the various processed HFR current anomalies, computed for the 54 collocated grid points.

\begin{tabular}{|c|c|c|c|c|}
\cline { 2 - 5 } \multicolumn{1}{c|}{} & HFR only & $\begin{array}{c}\text { HFR Ekman } \\
\text { Corrected }\end{array}$ & OI only & $\begin{array}{c}\text { OI and Ekman } \\
\text { Corrected }\end{array}$ \\
\hline $\begin{array}{c}\text { Angle (Complex Corr) in } \\
\text { degrees }\end{array}$ & $-9 \pm 16$ & $-4 \pm 17$ & $-3.7 \pm 16$ & $-2 \pm 15$ \\
\hline $\begin{array}{c}\text { Magnitude } \\
\text { (Complex Corr) }\end{array}$ & $0.64 \pm 0.16$ & $0.65 \pm 0.14$ & $0.7 \pm 0.13$ & $0.72 \pm 0.12$ \\
\hline
\end{tabular}

\subsubsection{Hovmöller diagram of OI and Ekman Corrected HFR and Altimeter currents}

The 3-year Hovmöller diagram for both velocity component anomalies and instruments are examined for 7 grid points along a fixed longitude of $-122.33^{\circ}$ between the latitudes of $35.5^{\circ}$ and $37^{\circ}$ (Fig. 4.7). The HFR currents are processed following the OI and Ekman corrected method and are magnified by a factor of 1.7 to match the altimeter levels of variations. First, the Hovmöller diagrams display very similar patterns for altimetry and HFR currents, with current anomalies in the range [-20 25] cm/s. Compared to the meridional (v) component, the zonal $(\mathrm{u})$ currents have moderately less amplitude variations and for a fixed time present more heterogeneity in the latitudinal response, with the point around $36^{\circ} \mathrm{N}$ being more energetic. The zonal currents also behave quite periodically with extrema 
between April and July. The RMS in $\mathrm{u}$ is $4.39 \pm 0.58 \mathrm{~cm} / \mathrm{s}$ and the RMS in $\mathrm{v}$ is $5.58 \pm 0.69$ cm/s. The difference (Altimetry minus HFR) Hovmöller diagram shows a structure with high and low patches related to higher and lower values of altimeter geostrophic currents which is more emphasied for the meridional component.
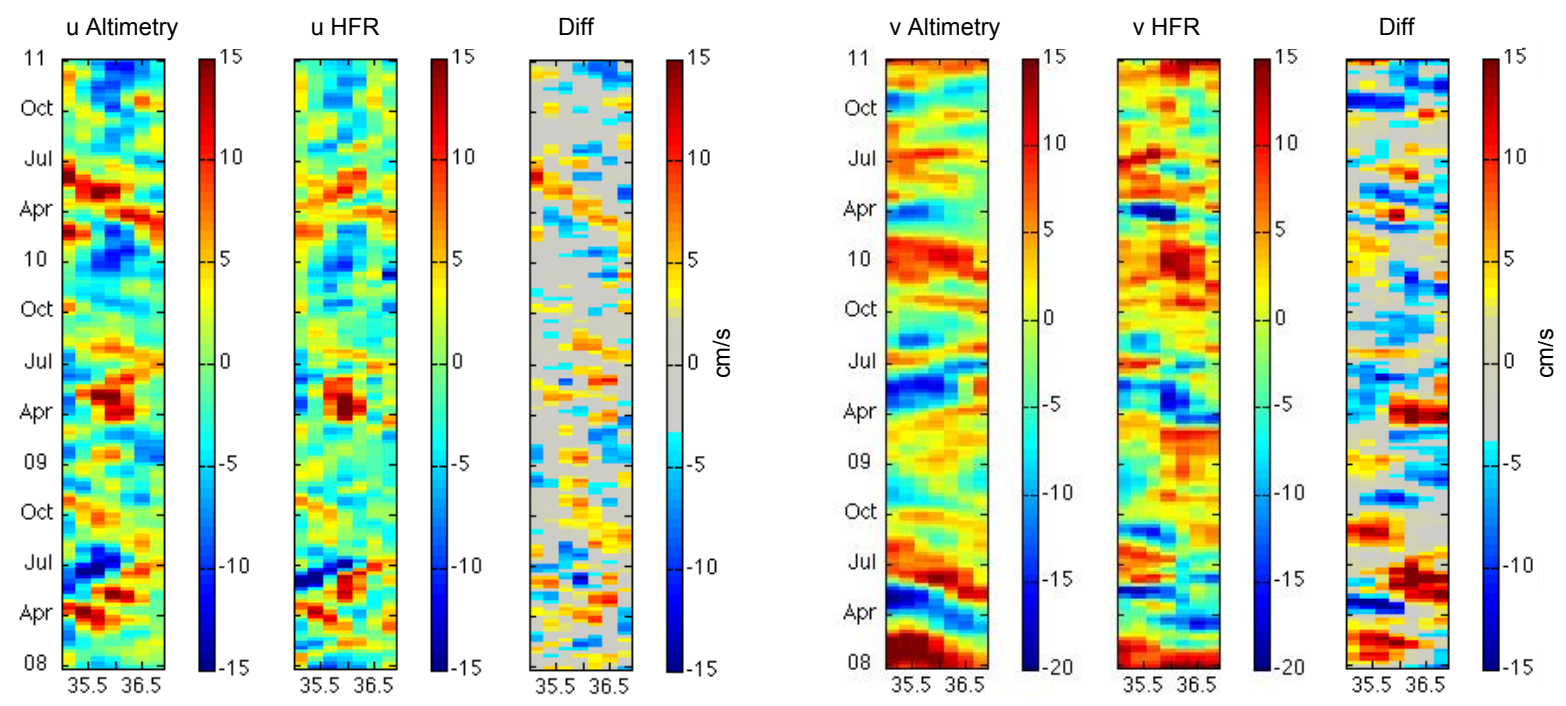

Figure 4.7: 3-year time series of zonal(left) and meridional (right) current anomalies for altimetry and OI Ekman Corrected HFR and their difference (ALT - HFR), along longitude $-122.33^{\circ}$

\subsection{Processing HFR from currents to Sea Surface Heights}

\subsubsection{Methodology}

As previously mentioned, the OI estimates directly from the HFR currents the nondivergent stream function $\psi$. If the field is geostrophic then $\psi$ and sea level SSH are proportional with a factor $g$, the gravitational acceleration, divided by $f$, the local Coriolis parameter: $\psi=\frac{g}{f} S S H$. The SSH field is inherently less noisy than the derived OI velocity field which are calculated from a derivation of the SSH $($ or $\psi): u_{O I}=-\frac{d \psi}{d y}, v_{O I}=\frac{d \psi}{d x}$. But this also implies that these SSH contain an unknown integration bias, that will be the same for each grid point of a weekly set, but different for each weekly set, because they are 
estimated from currents. In this section the synthetic SSH will be computed with the OI Ekman Corrected method that was found to be more appropriate in the previous analyses. Here the weekly altimetry MSLA (Merged Sea Level Anomalies) from AVISO/DUACS over the years 2008 till 2010 are analyzied, with the corresponding weekly HFR synthetic SLAs, on the $1 / 3^{\circ} \times 1 / 3^{\circ}$ grid for the Californian coastal region. The mean of the sea level time series is removed from both data sets. To adjust for the unknown bias for each weekly HFR sea levels (derived from the OI method), an estimate of the bias over the complete region is computed, and then subtracted. The bias for each week is computed by taking the mean of the difference between the HFR adjusted by a factor 1.7 and MSLA sea levels, over the entire region.

\subsubsection{Results}

For the year 2008, a movie for the weekly time series of both sets was created and is presented as snap shots in Fig. 11 of Roesler et al. (2013 [65]) with a sampling of every 6 weeks. After August 2008, the field extends to the north, because the coverage of the 6-kmHFR grid increases. The time evolution of these two fields for the year 2008 shows excellent agreement: the formation and development of eddies is nearly identical in both series. The time series has now been extended over 3 years. To quantify this relationship the SLA time series for the 157 weeks, for each grid point, in the sub-region $34^{\circ}$ to $38^{\circ}$ available for the whole 3 years, have been retrieved for both HFR and altimetry. The correlations for the 81 grid points between the two SLA time series (Fig. 4.8 left) are excellent and is over 0.8. Note that the coverage for the SLA is slightly larger than the one for the current anomalies, because the derivation required to obtain the OI currents reduces their availability around the region boundaries. This spatial correlation has been observed to vary from year to year, and was larger for the year 2010, which is also connected with lower winds.

The 3-year Hovmöller diagram for both SLAs along longitude -122.33 for 10 grid points, displayed in (Fig. 4.8 right), shows very similar SLA behavior for both datasets. 

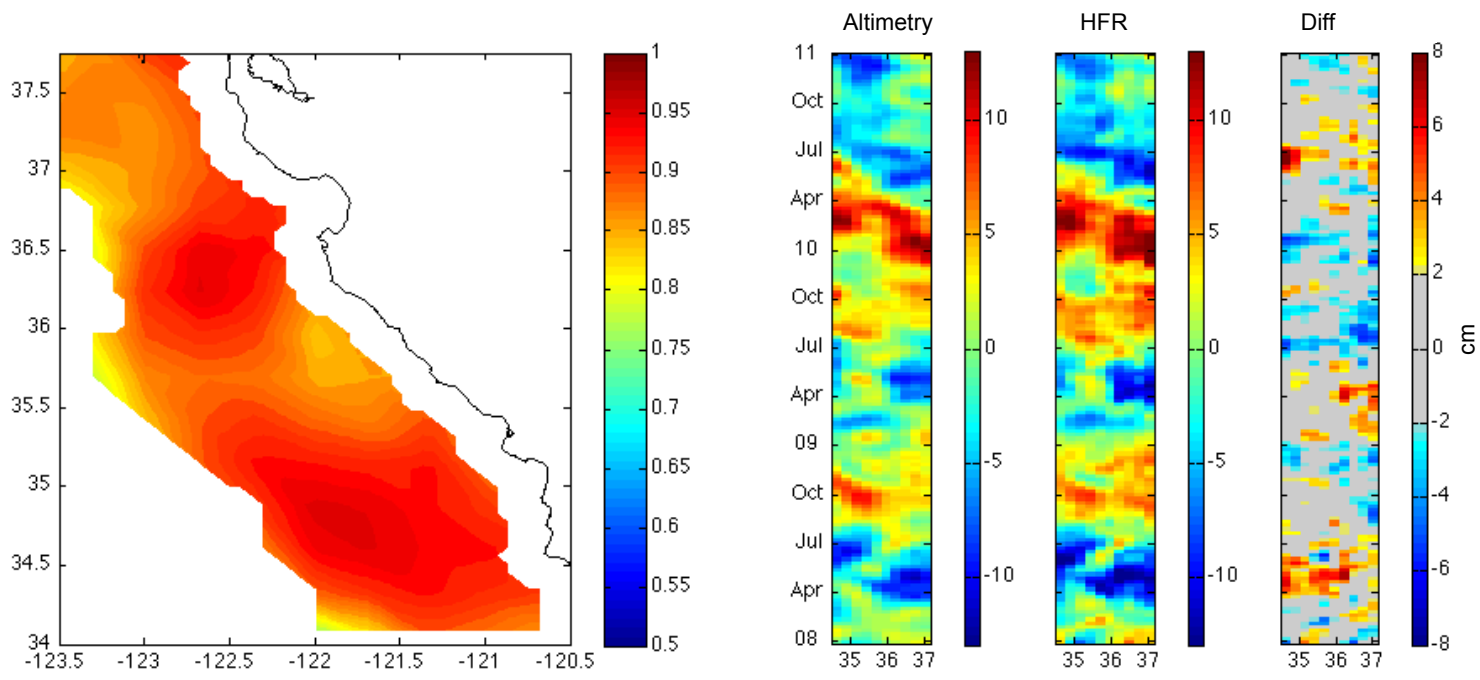

Figure 4.8: (Left) 3-year mean correlation in SLA between altimetry and OI Ekman Corrected HFR. (Right) 3-year time series of the SLA for altimetry and OI Ekman Corrected HFR and their difference (ALT - HFR), along longitude -122.33. ${ }^{\circ}$

Overall besides some phase shifts, the altimeter SLA seem to deamplify the lows and highs relative to HFR synthetic SLA. The averaged RMS for the time series is $1.8 \pm 0.25 \mathrm{~cm}$.

This chapter developed a methodology to retrieve the geostrophic part of the HFR currents, by averaging the HFR currents in time, removing the Ekman current, and applying an OI to force geostrophy. These results, although on a large time scale and low resolution spatial grid, suggests that the HFR synthetic SSHs can be used as a proxy for the altimetric measured heights in the open ocean where the waveforms are, usually, not distorted due to proximity to land. Next the methodology is improved and evaluated to fit the characteristics of an altimeter track. 


\section{Chapter 5}

\section{Methodology along an offshore altimeter track parallel to the coastline}

\subsection{Introduction}

The methodology to retrieve the geostrophic signal from the HFR currents is tested and improved to fit the characteristics of an offshore Jason-1 altimeter along track measurements. This track offers $750 \mathrm{~km}$ of altimeter data that are coincident with the 6 -km HFR currents. Using this offshore altimeter track, where the altimeter data are trusted, provides a testbed to explore the validity of the technique at the $1-\mathrm{Hz}$ level (i.e. a sampling every $6 \mathrm{~km}$ ), and at the altimeter time of passage. This will be a marked improvement relative to the large time and space scales that were investigated in the previous chapter. In this section the comparison of the HFR/Altimetry datasets for the year 2010 is studied in detail.

\subsection{Altimeter Track Location}

Jason-2, after its launch in June 2008, shared the same orbit as Jason-1 for calibration purposes. Jason-1 assumed a new orbit, the interleaved orbit, midway between its original ground tracks after March 2009 (until March 2012, when it was placed in a geodetic orbit). With Jason-1 in its interleaved orbit there is the opportunity to use the Pass 206 (P206), which is very conveniently located for this study. Jason-1 P206 runs parallel to the California coastline with on offshore distance of at least $50 \mathrm{~km}$, in a region where the altimeter measurements are reliable, that is not affected by the nearshore coastal problems. Fig. 5.1 shows the location of J1 P206 in relation to the coastline and the HFR currents coverage. 
P206 is a descending track; the altimeter is travelling north to south. Fig. 5.1 also presents the repartition of the number of hourly 6-km resolution HFR data samples for the year 2010. The along track segment between lat $34^{\circ}$ and $39^{\circ}$, is sampled almost all year around, and is chosen for the comparison. This track offers $750 \mathrm{~km}$ of continuous along track altimeter data that are coincident with the 6-km HFR currents.

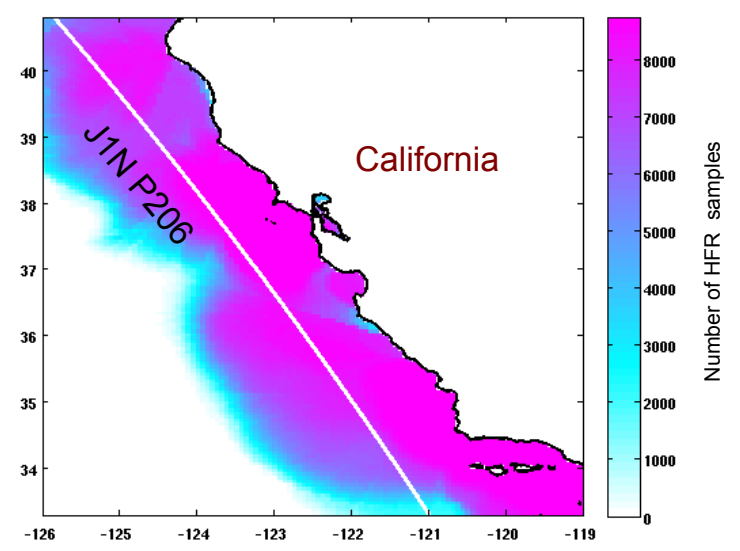

Figure 5.1: 6-km resolution HFR data coverage for the year 2010. The color indicates the number of HFR hourly samplings over the year. The white line is Jason-1 P206 in its interleaved orbit

\subsection{Data Processing}

\subsubsection{Processing of the altimeter data}

The along-track altimetry data used in this section are from the GDR product for Jason-1 (version C), obtained from AVISO. The 1-Hz MLE-4 data rate (equivalent to a resolution of approximately 6-7 km) have been reprocessed, using the $20-\mathrm{Hz}$ data rate to remove outliers following specific criteria to generate a good quality estimate of SLA.

- The editing criteria provided in the altimetry handbook have been applied to the auxiliary corrections so that they are edited when they are beyond a certain threshold. 
- The dual-frequency rain flag algorithm based on MLE3 sig0 (Tournadre 2004 [71]) is used to detect rain events.

- In addition, specific measurements at the $20-\mathrm{Hz}$ level have been remove: with sig0 larger than $16 \mathrm{~dB}$ : to remove waveforms contaminated by low winds, or surface slicks; With SWH higher than $5 \mathrm{~m}$, which increases the noise level in the SLA data.

- All the usual corrections to the range measurements have been applied, to obtain the SLA.

- The extreme outliers have been removed using a Lee-filter (Lee 1980 [55]) at the 20-Hz level, with a 3 sigma boundary editing.

- The data are interpolated at equally sampled reference points along a nominal ground-track. Each along-track can deviate from 0 to $1 \mathrm{~km}$ from a reference ground track. The nominal ground-track for 2010 has been selected as the along track that falls in the middle of the cluster of the 2010 along tracks.

- Since the noise level limits the ability to observe the smaller along-track wavelengths, an along track spatial low-pass filtering, using a minimum $30 \mathrm{~km}$ (91 points) boxcar window is applied before data analysis. The altimeter SLA have been sampled at the $1-\mathrm{Hz}$ data rate, which is also the resolution of the HFR currents. The mean of the 2010 time series is removed from the SLA dataset, so that the sea level variability will be consistent with the one derived from the HFR currents.

\subsubsection{Processing of the HFR currents}

In this section the 6 -km resolution gridded HFR surface currents are used, because there are no 2-km resolution HFR currents that reach P206. Altimetry maps the vertically integrated SSH that can be related to the geostrophic flow, whereas HFR data gives us a surface total velocity and includes ageostrophic processes that need to be removed to compare 
the two data sets. In this study an OI is chosen to retrieve the SSH from the total velocity surface measured by the HFRs. The OI requires an analysis of the time and spatial scales of the coastal oceanic features.

The methodology is explained in detail in section3 of Roesler et al. (2013 [65]) for an ascending altimeter track approaching the coastline, where the spatial covariance scales decrease from about $100 \mathrm{~km}$ offshore to $10 \mathrm{~km}$ near-shore. The oceanic spatial scales have been computed from the analysis the HFR currents over the year 2008. Jason-1 P206 is located in a dynamic offshore region, further than $60 \mathrm{~km}$ offshore. Statistically the size of the eddies are about $60 \mathrm{~km}$ wide and squeezed between them there could be upwelled coastal filaments or jets, with a width of $50 \mathrm{~km}$ or less, and flowing with speeds that could reach $50 \mathrm{~cm} / \mathrm{s}$. The OI will be applied by using only one spatial covariance scale chosen to be $30 \mathrm{~km}$. This spatial scale has been selected as a compromise between too much smoothing by using too large of a spatial scale and not being able to track the small features, and not enough so that the eddy structure will not be adequately detected. The velocity noise error for the HFR currents is assumed to be constant $e=15 \mathrm{~cm} / \mathrm{s}$.

Before applying the OI, the HFR surface currents are averaged over 3-days to remove the short-term ocean variability due to winds and other factors, which are not in geostrophic balance. Also, in case there is a gap in the HFR time series, the tides estimated from the $10 \mathrm{~km}$ OSU Pacific Ocean tide model are removed. This data will generate one set of sea level heights derived from the HFR currents. Another set will be produced by removing an estimate of the Ekman Currents to the HFR currents, before applying the OI. The Ekman currents are estimated using the 4-hour NARR NCEP reanalysis wind data distributed on a $25 \mathrm{~km}$ resolution grid. This wind data is interpolated hourly on the $6-\mathrm{km}$ HFR grid. The Ekman coefficients are chosen to be constant along the track, with $\alpha_{E}=1$ for the amplitude and a deviation $\varphi_{E}$ of $60^{\circ}$ to the right of the wind, that were estimated from the previous analysis (Chap4.2.1). Note that the closer the input set is in geostrophic balance, the closer the OI derived sea levels should be to the true sea surface dynamic heights. This fact will 
be tested to check how much improvement is obtained from the second set.

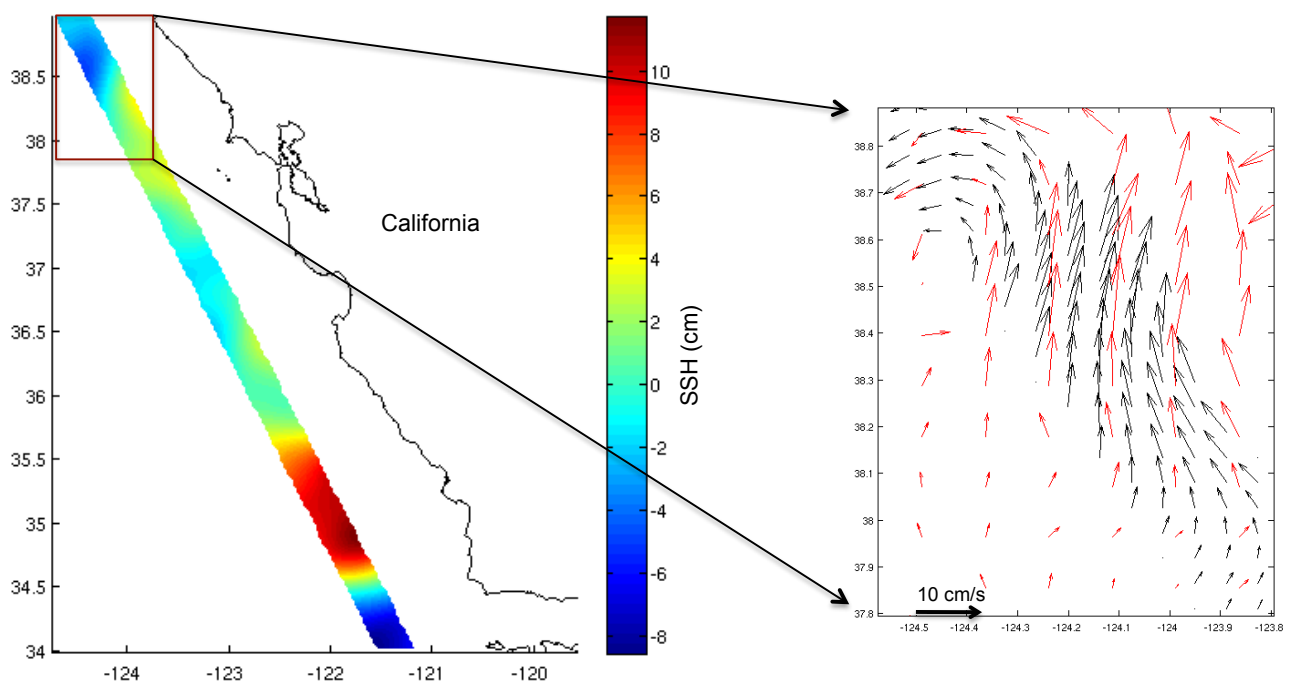

Figure 5.2: OI output. (Left) OI retrieved SSH around J1 P206. (Right) Corresponding 6-km OI output currents in black; HFR input currents in red, every other $6 \mathrm{~km}$

Only a 40-km-wide strip around P206 is inverted. Fig. 5.2 shows one SSH map derived from the HFR currents on April 20, as well as the corresponding output OI currents (in black) and the original input HFR currents (in red). The OI mapping error is relatively constant throughout the whole year and throughout the strip. The residuals of the optimally interpolated field should be consistent with the assumed error variance of the data. Over 2010, we find that the root mean square (RMS) difference of the HFR and the OI mapped velocities in the $\mathrm{u}$ and $\mathrm{v}$ component are $3.6+/-0.93 \mathrm{~cm} / \mathrm{s}$ and $3.9+/-0.75 \mathrm{~cm} / \mathrm{s}$ less than the estimated error variance of $15 \mathrm{~cm} / \mathrm{s}$. Recall that the HFR synthetic sea surface heights contain an unknown bias, because the OI cannot determine the absolute SSH. The mean of the HFR synthetic SSH time series over 2010 is removed to obtain the HFR SLA. 


\section{$5.4 \quad$ Results}

\subsubsection{Along track SLA}

Fig. 5.3 displays the altimeter SLA filtered with a boxcar window of $45 \mathrm{~km}$ for the 36 Jason-1 cycles repeating every 10 tens days for the year 2010. Cycle C315 at the end of July is unavailable, and the altimeter data for C296, on January 21, with our stringent outliers removal for high SWH are invalid. This leaves 34 cycles, which is just above the minimum number of 30 (central limit theorem) required so that the cycle measurements errors can be assumed to have a population with normal probability density function. In Fig. 5.3 for each cycle, the SLA are demeaned over the along track, so that each cycle can be viewed in a $[-15$, 15] $\mathrm{cm}$ window. This mean, that is being removed, and which is calculated as the mean over the section of the along track between $34^{\circ}$ and $39^{\circ} \mathrm{N}$ for each cycle, represents the seasonal variation of the altimeter SLA. The SLA seasonal variation (Fig. 5.4) during the year 2010, are typical for the California coastal region, and are characterized by higher sea levels in winter; a rapid decrease in spring an low sea levels lasting until mid-summer, here with an extreme low event in July; and finally a steady increase of sea levels after August.

Two HFR SLA datasets are also presented in Fig. 5.3, one without (raw HFR SLA )( in red) and the other with the Ekman correction (Ekman corrected HFR SLA) ( in blue). The datasets are demeaned over the along track, for each cycle. This has the effect of removing the unknown bias contained in each HFR SLA set, since the OI processing cannot determine the absolute sea surface heights. No amplification factor has been applied to the HFR SLA. The first noticeable fact is that the quality of the HFR SLA are remarkably improved after the Ekman correction is applied, relative to the assumed true SLA given by altimetry. The cycles C300 (Mar-02), C306 (Apr-30) and C330 (Dec-24) are good representative cases of the amelioration. The strong linear deviation observed in some of the raw HFR SLA, such as C295, C300, C330, can easily be explained. To simplify, assume a steady wind field that generates an Ekman current of $5 \mathrm{~cm} / \mathrm{s}$. It can add an additional $5 \mathrm{~cm}$ in sea level height 

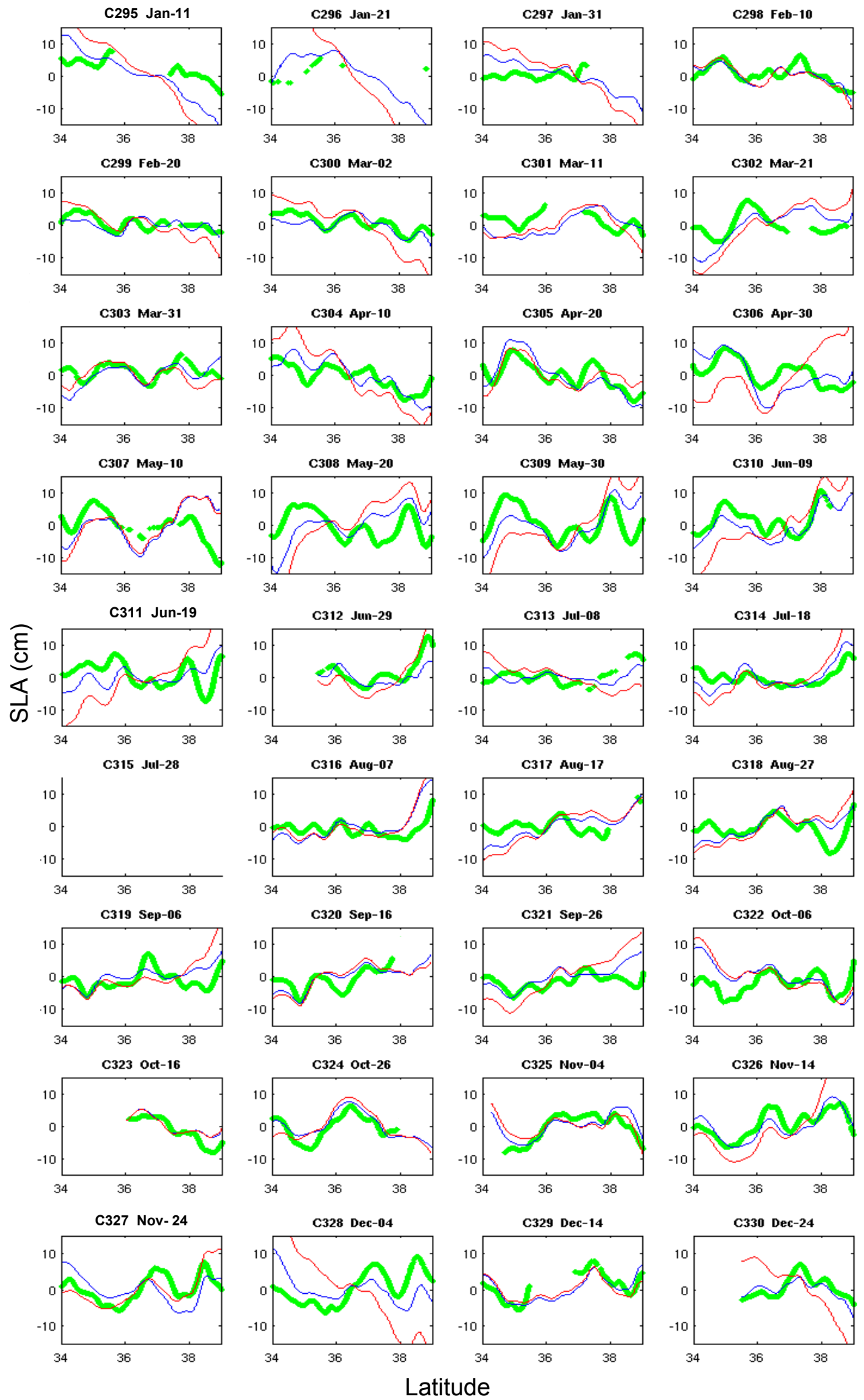

Figure 5.3: Altimeter and HFR sea levels along P206; Jason-1 SLA smoothed with a 45-km low pass filter (green); Raw HFR SLA (red); OI Ekman Corrected HFR SLA (blue) 
every $100 \mathrm{~km}$, which is seen as a linear trend superimposed on the true SLA signal. The obvious case C295 (Jan-11) presents clearly a strong decreasing linear trend in the raw HFR SLA about the level just mentioned. It is reduced, after the Ekman correction, but not completely resolved, as there is still a linear residual. On the other hand, the large SLA linear trends observed before the Ekman Correction for the cycles C300 (Mar-02) and C330 (Dec-24) are satisfactorily eliminated after. These examples confirm that the problem of reducing the wind driven components is not straightforward.

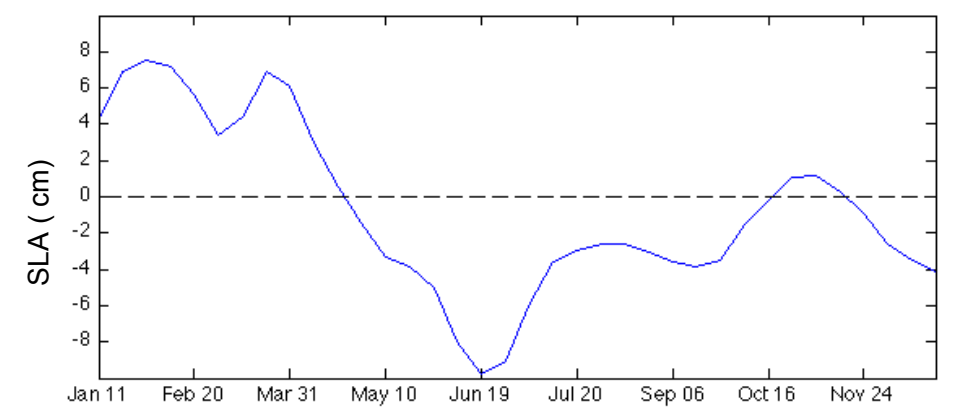

Figure 5.4: Seasonal variation of the altimeter SLA averaged along the Californian portion of P206 during 2010

\subsubsection{Spectral Analysis}

Fig. 5.3 also reveals that in this region the 6-km HFR SLA contain less high frequency levels than the altimeter data. This is a limitation for the purpose of this study, because in order to compare the two products, the altimeter along-track data need to be filtered to the same level of smoothness as the HFR SLA. This implies that the detection of the small scales oceanic or atmospheric perturbations that affect the altimeter measurements may be left unnoticed using the HFR currents as a validation reference. This threshold on the tractable spatial scale is limited by the frequencies in the HFR currents, as well as the weakness of the perturbation. To better understand the difference in the spatial spectral characteristics 
between the altimeter and the Ekman corrected HFR SLA, the power spectral density (PSD) have been computed along the California portion of P206 (Fig. 5.5). For both datasets, the average spatial spectrum over the year 2010 has been computed using a sampling rate of $6 \mathrm{~km}$ (the resolution of the HFR) and restricted to the $750 \mathrm{~km}$ along track segment. Two spectra for the altimeter SLA are obtained, one directly from the 1-Hz data (in black); the other after applying two successive filters to the 20-Hz data, first a running average over 91 points (i.e. $30 \mathrm{~km}$ ), then another over 150 points $(45 \mathrm{~km})$ and finally resampled to $1-\mathrm{Hz}$.

The Ekman corrected HFR SLA have been used. Its spectrum (in blue) shows that the HFR SLA noise level starts just below wavelengths of about $50 \mathrm{~km}$, unlike the $1-\mathrm{Hz}$ altimeter SLA noise level that starts below $30 \mathrm{~km}$. The altimeter white noise due to geophysical and instrument noise will vary from one region to another, and will also depend on the processing techniques. Statistically for this location, the eddy sizes are about $60 \mathrm{~km}$ but squeezed between them there could be coastal filaments or jets, with a maximum width of $50 \mathrm{~km}$. So part of the altimeter SLA content at the mesoscales is of dynamical content. Being able to separate the signal from noise is a delicate issue. For wavelengths higher than $50 \mathrm{~km}$ the HFR spectra present a steeper increasing slope with lower spectral content, until the larger wavelengths of $200 \mathrm{~km}$. The spectrum from the filtered altimeter SLA is very similar to the HFR spectrum with slightly more energetic content. This filtered altimeter SLA dataset will be the one used to compute more in depth statistics between the HFR/altimeter SLA.

\subsection{Statistics RMS/ Correlation}

To quantify the amelioration in the quality of the HFR SLA, the RMS between the altimeter SLA and both HFR SLA datasets are computed for each cycle, together with their correlation. The results for each cycle are presented in Fig. 5.6. The RMS computed using the raw HFR SLA are generally higher than the RMS derived from the Ekman Corrected HFR SLA. Over the 34 cycles the mean RMS $=5.74+/-3.4 \mathrm{~cm}$ for the first set, compared to 


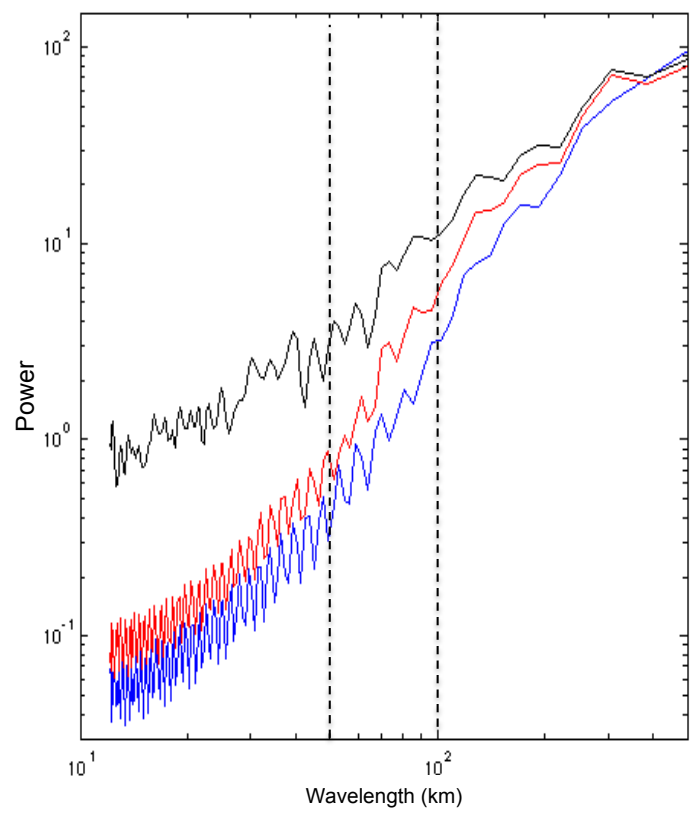

Figure 5.5: Spectrum for the Ekman Corrected HFR SLA (blue); for the Jason-1 at the 1-Hz level (black) and filtered with a boxcar window of $50 \mathrm{~km}$ (red)

$\mathrm{RMS}=3.51+/-1.4 \mathrm{~cm}$ for the other. This represents an improvement of $40 \%$ of the RMS after removing the Ekman current. Also shown on Fig5.6 are the standard deviations (STD) of the altimeter SLA along the track for each cycle. They indicate cycles that have large SLA variations along the track. For instance the altimeter SLA have STD higher than $4 \mathrm{~cm}$, from April until the end of June. This corresponds to the upwelling season, with strong winds, the generation and strengthening of eddies as well as the intrusion of upwelled filaments. During this period the RMS from the raw HFR SLA are also very high with values above $8 \mathrm{~cm}$. But the RMS from the corrected Ekman do not follow this trend, and are usually similar or less than the altimeter STD (except on May 10th). The meaningful correlations above 0.6 between the HFR and altimeter SLA are also plotted on this Fig. There are 17 cycles with such correlations and only 2 cycles (C312 June -29 and C327 Nov-24) when the correlation is better by calculating it from the raw HFR SLA, but those are also cases when the RMS for the two HFR SLA datasets are similar. The mean correlation, over the 17 cycles that 
have a correlation larger than 0.6 , is computed for both HFR SLA datasets. It is $0.63 \pm 0.3$ using the raw HFR SLA and the one derived from the Ekman corrected HFR SLA is 0.73 \pm 0.11 . There is an amelioration of $17 \%$. For these 17 cycles the RMS lowers to $2.51 \mathrm{~cm}$.

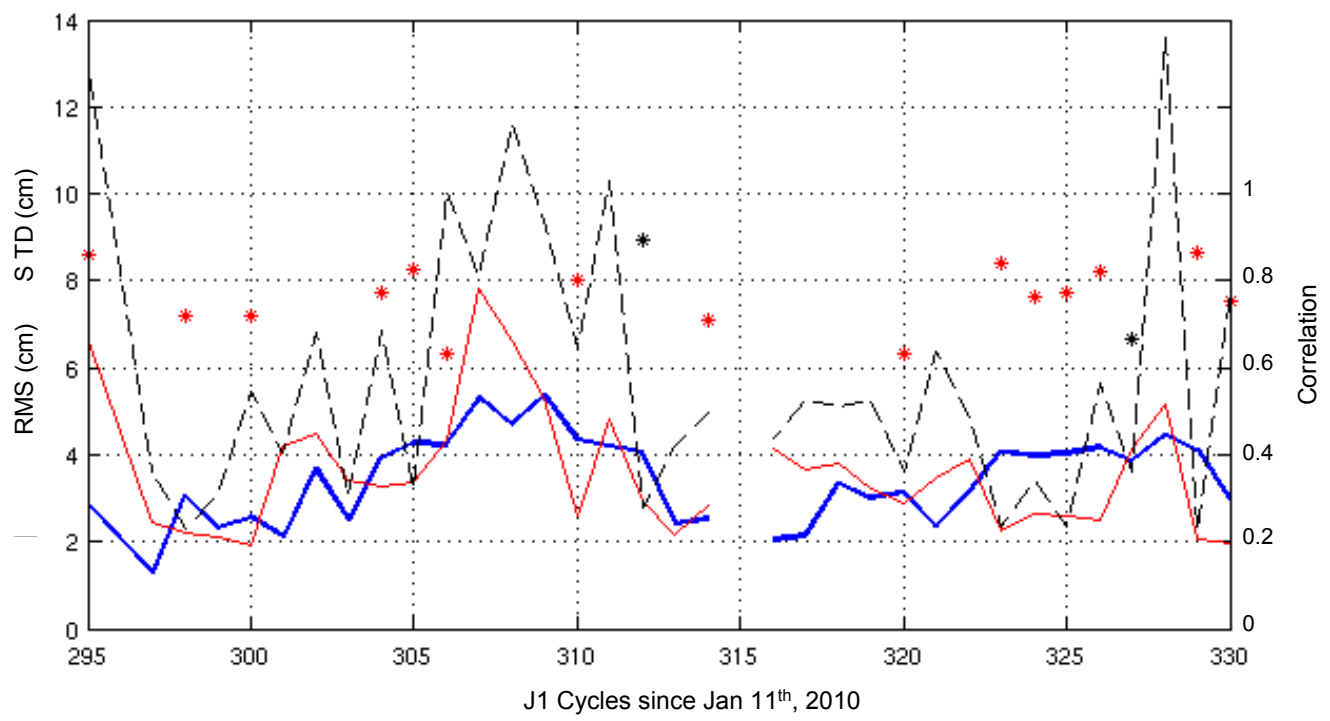

Figure 5.6: Statistics for the 2010 Jason-1 cycles. STD of the altimeter SLA (blue). RMS between the raw HFR and altimeter SLA (dotted black). RMS between the Ekman Corrected HFR and altimeter SLA (red). Correlation between Ekman corrected HFR and altimeter SLA above 0.6 (red stars). Two cases are better correlated using the raw HFR SLA (black stars)

\subsubsection{Time Averaging choice for the HFR currents}

So far a 3-day averaging of the HFR currents has been applied to remove the inertial currents. The question remains whether a smaller time averaging would contain more high frequencies spectral information and improve the relationship with the altimeter measurements, which are taken at one specific time. To answer this question, the Ekman corrected HFR SLA are processed using 5 different time averaging: 3-day, 2-day, 1day, 15-hour and 1-hour. Table 5.1 displays the statistical results in terms of mean RMS and correlation between the various time-averaged HFR datasets and altimeter SLA over the cycles. The 3-day averaging provides the best statistics with an $\mathrm{RMS}=3.5 \pm 1.4$ and correlation $0.5 \pm$ 
0.3 and the 1-hour the lowest with an $\mathrm{RMS}=6.5 \pm 3 \mathrm{~cm}$ and correlation $0.32 \pm 4$.

One of the problems is that the Ekman correction is difficult to apply for the 1-hour averaging, because this is done using the wind at that time, which may not be suited as the theoretical Ekman correction assumes a steady wind condition. So that even if the 1-hour SLA datasets contain slightly higher frequency features, they are superimposed on unrealistic SSH patterns caused by short-term surface wind drifts that are difficult to remove. Although there is not much significant difference between the 2-day and 3-day averaging statistics, the 3-day time average is chosen in this study.

Table 5.1: RMS and correlation between the HFR/Altimeter SLA for various time-averaging of the HFR currents

\begin{tabular}{|c|c|c|c|c|c|}
\cline { 2 - 6 } \multicolumn{1}{c|}{} & 3-day & 2-day & 1-day & 15-hour & 1-hour \\
\hline RMS (cm) & $3.5 \pm 1.4$ & $3.8 \pm 1.6$ & $3.9 \pm 2$ & $4.7 \pm 2.2$ & $6.5 \pm 3$ \\
\hline Correlation & $0.5 \pm 0.3$ & $0.49 \pm 0.3$ & $0.48 \pm 0.31$ & $0.45 \pm 0.34$ & $0.32 \pm 0.4$ \\
\hline
\end{tabular}

\subsection{Influence of the wind history}

The HFR surface currents contain some wind-driven drift, so there must be a relationship between the wind history and the quality of the geostrophic HFR SSH. This relationship is investigated in this section.

\subsubsection{Wind anomaly and quality of the HFR SLA in terms of RMS}

The wind tensor $\|W\| W$ anomaly is interpolated along the altimeter track and averaged over 3 days, for each cycle. The mean magnitude and mean direction of the wind tensor anomaly are calculated over the $1000 \mathrm{~km}$ segment, for each cycle. This provides and 
indication for the wind condition at the altimeter time of passage. Fig. 5.7 displays the wind tensor anomaly magnitude and direction, together with the RMS between the HFR and altimeter SLA. First lets look at the RMS derived from the raw HFR SLA. These RMS are higher when either the wind tensor anomaly in direction or/and speed is large. This behavior is also seen in the RMS computed from the Ekman corrected HFR, but with more subtle variations. Clearly, the winds play a role in the quality of the extraction of the geostrophic signal from the HFR currents.
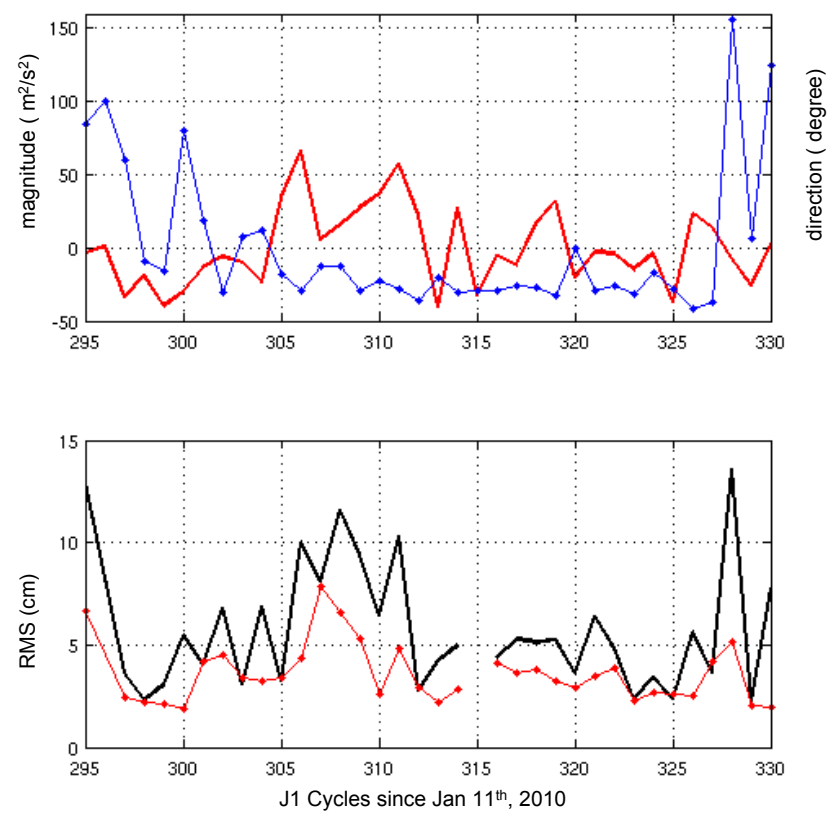

Figure 5.7: SLA and wind tensor anomaly Statistics for each 2010 Jason-1 Cycle. Top: mean averaged over the segment of the wind tensor anomaly magnitude (red) and direction (blue). (bottom) RMS between the raw HFR and altimeter SLA (dotted black). RMS between the Ekman Corrected HFR and altimeter SLA (red).

\subsubsection{Concerned only with the across-track Ekman current}

In this section one example will provide a better understanding of the influence of the wind on the methodology. The case C326 (November 14) is a situation when at each along track location, the wind direction and speed stay relatively constant, but vary with latitude 
(Fig. 5.8). So locally, the steady wind condition is valid. The theoretical Ekman current will be $60^{\circ}$ to the right of the wind tensor, for the HFR current in this California coastal region, as estimated in the previous chapter. Because the altimeter can only measure the across track geostrophic currents, only the projection of the Ekman current perpendicular to the track are of concern and will influence the quality the HFR geostrophic current retrievals. At the California latitudes, Jason-1 P206 is inclined $30^{\circ}$ to the left of the North direction and runs almost parallel to the coastline. The wind variability is mainly in the north-south direction, and with a rotation of $60^{\circ}$ to the right of the wind the Ekman current will be directed mainly across-track. Thus, their influence cannot be neglected for this specific track P206. For the case (C326) the across-track Ekman current will be larger as we move to the North, because not only the wind tensor magnitude increases but also the direction changes. This can be related to the strong linear deviation observed in the raw HFR SLA after the latitude $37^{\circ}$. Since locally the steady wind condition is valid, the Ekman corrected HFR SLA match the altimeter data.
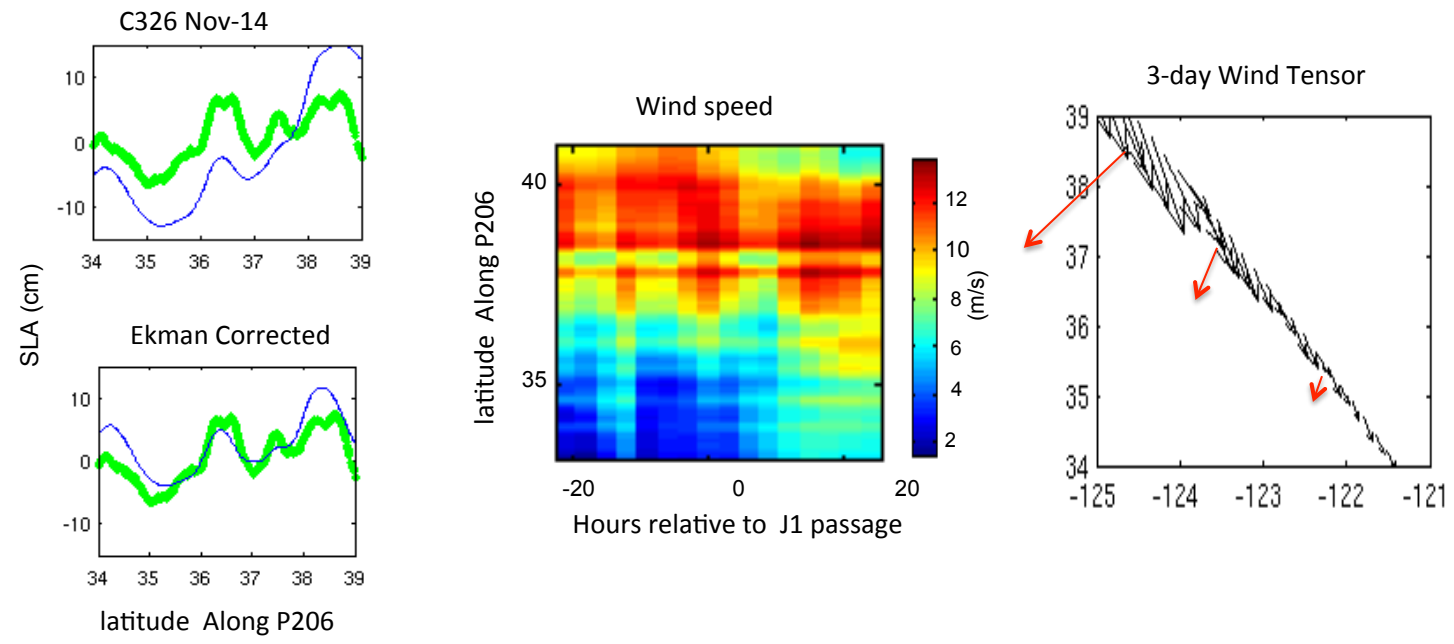

Figure 5.8: Case study for C326. (Left) Altimeter (green) and HFR (blue) SLA for (top) Raw HFR SLA (bottom) OI EKman Corrected HFR SLA. (Middle) Hourly wind speed along the altimter track. (Right) 3-day averaged wind tensor on P206 (black); Ekman Current (red) not to scale 


\subsubsection{Wind history: 4 general case studies}

For each cycle, the mean and STD as a function of latitude, along P206, of the wind direction and speed over 3 days, can help characterize the wind behavior. But these indices have no indication of when or how the wind changes in time. The use of the daily averaged wind over the California region will provide a hint about their time evolution. Next, four typical scenarios of wind event are discussed as well as their effect on the quality of the HFR SLA.

(1) Constant wind speed and direction

The cases when the wind is not changing over time (Fig. 5.9) satisfy the requirement of the steady wind assumed for the theoretical Ekman drift. These events are typical of upwelling conditions, with strong winds blowing to the south. They can be observed on April 30, May 30, June 19, July 18, Sep 26 and Nov 14. For all these dates, the Ekman Corrected HFR SLA resolves a lot of the ageostrophic surface wind drift, and is a much better fit to the altimeter SLA; especially considering that during these events, the sea level variations are large along the track. Looking more closely at our statistic and time indicators, can explain some of the misfits. For example on April 30, and July 19, the winds are overall higher than the other cases, with winds larger than $10 \mathrm{~m} / \mathrm{s}$. The consequence is that an incorrect estimate of the Ekman coefficients or winds will have a larger error on the correction, since the Ekman current varies as a function of the wind speed squared.

(2) Winds turning and high speed

On January 11, there is an atmospheric perturbation passing through the California coastal ocean. Initially on January 10, the region is separated into a northern section above $37^{\circ}$ where the winds are blowing to the north, and a southern section with southward winds. Then as the perturbation travels, on January 12, all the winds are blowing more consistently to the north. The winds also change in speed, with 
increasing speeds in the northern area.

This is a case where in the northern section there is a clear deviation in direction from the 3 -year mean direction, which is around $-50^{\circ}$. This section of northward blowing winds also has wind speeds that are increasing throughout the 3 days to reach $15 \mathrm{~m} / \mathrm{s}$. These indicate complications with the Ekman correction. Furthermore the winds in the southern section are slowly turning to the north. There is a perturbation passing by. Indeed, even if the Ekman corrected HFR SLA are improved relative to the raw HFR SLA, they still contain some large wind drift residuals.

(3) Winds turning but decreasing in speed after the passage of the altimeter October 6, October 26 and November 4 are cases when the STD in direction are large. But the medium wind field before the passage of the altimeter has been blowing in a steadily way to create a steady Ekman response. Progressively the winds decrease, so that even if they turn, the induced wind drift error becomes small. For those cases the Ekman Corrected SLA fits the Altimeter SLA, except in the boundary parts where the altimeter could still be in error (maybe there are some outliers not removed and/or there are some smoothing effect at the boundaries).

(4) Low winds turning but increasing in speed after the passage of the altimeter These cases are represented by the cycles of August 27, and December 4. The winds are low before the passage of the altimeter. But suddenly increases to a constant speed and direction. The Ekman response takes several hours to a day to arrive at its steady state condition. In these cases there are still some wind driven residuals in the Ekman corrected HFR SLA.

Studying the wind history at the hourly scales also explains why a smaller-time averaging would give a better estimate in certain cases. 
a)

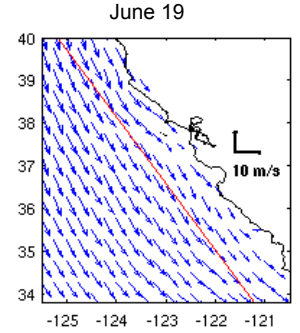

b)

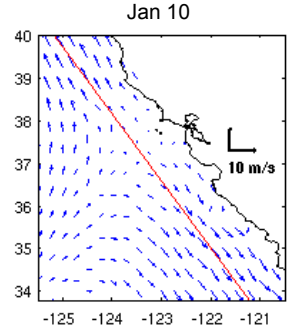

c)

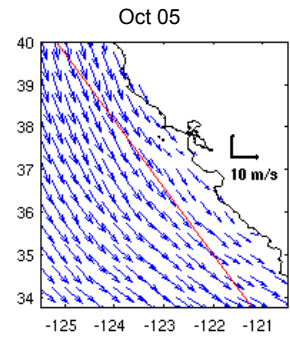

d)

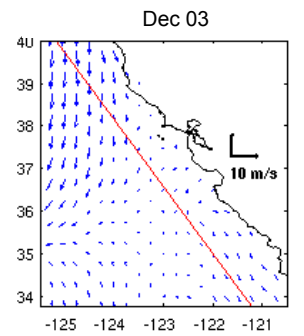

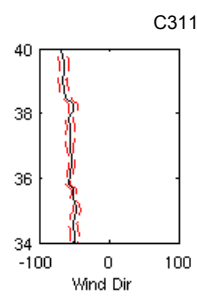

311 June-19

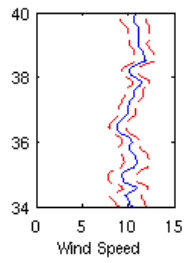

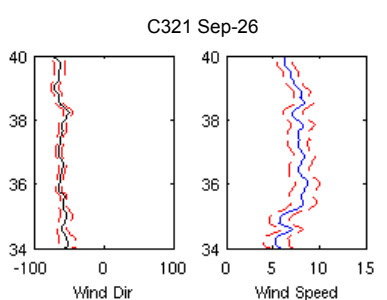

Jan 12
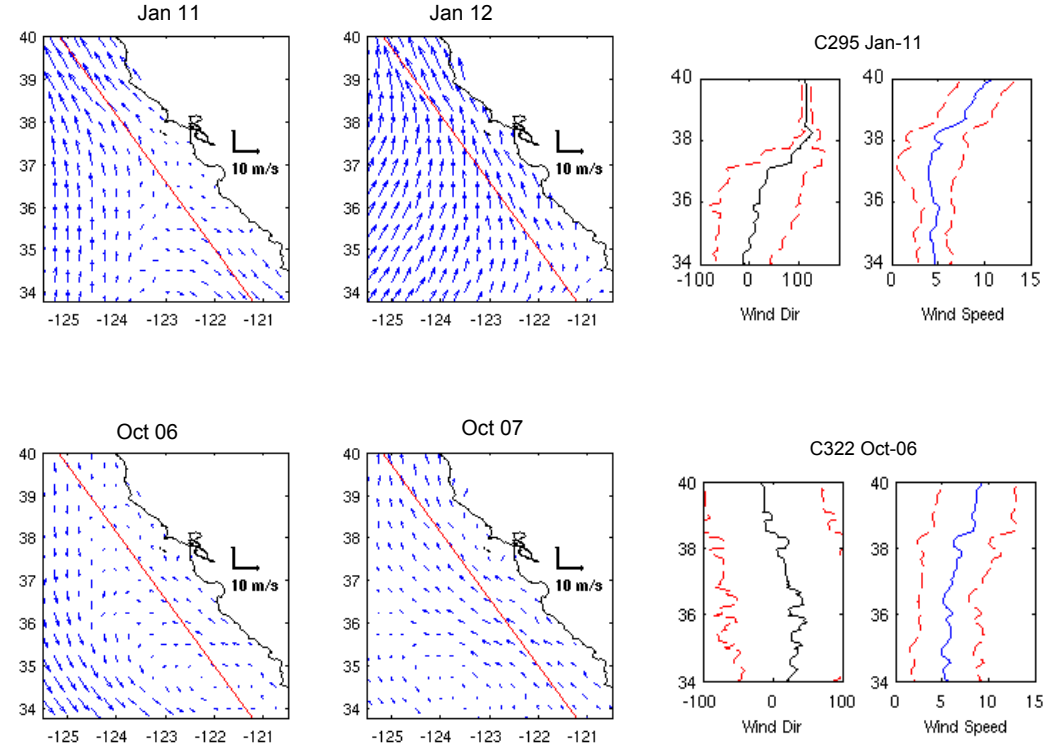
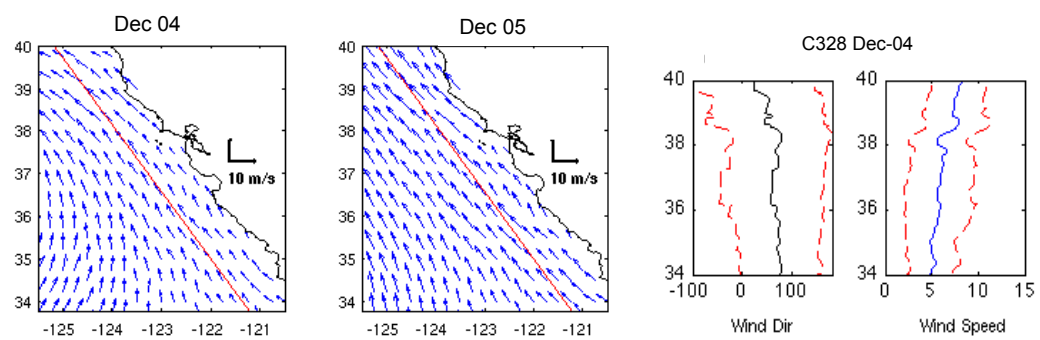

Figure 5.9: a) (Left) Daily winds centered at the time of passage for C311. (Right) 3-day (centered at the time of passage of the altimeter) mean and STD of the wind direction and magnitude along P206 for 2 similar cases C311 and C321. (b, c, d) Three consecutive daily winds, with the middle one centered at the time of passage of the altimeter. (Right) 3-day mean and STD of the wind direction and magnitude along P206.

\subsection{Using an SST image to decide between HFR or Altimetry SLA as a closer estimate}

The fact that the two HFR/altimetry sea level sets may have a phase shift between 
them arises from the filtering processes. What is more of a concern is when the altimetry SLA have a strong signal that is not observed by the HFR SLA. For instance on June 29, C312 the SLA variations between $38^{\circ}$ and $39^{\circ} \mathrm{N}$, are much larger. There are several possible reasons: The Ekman correction was inappropriate: too much/not enough; the 3-day average reduces too much of the variability present at the time of passage of the altimeter track. The event captured by the altimeter could be short lived, or drifting away; the HFR currents simply do not capture the signal observed by the altimeter.
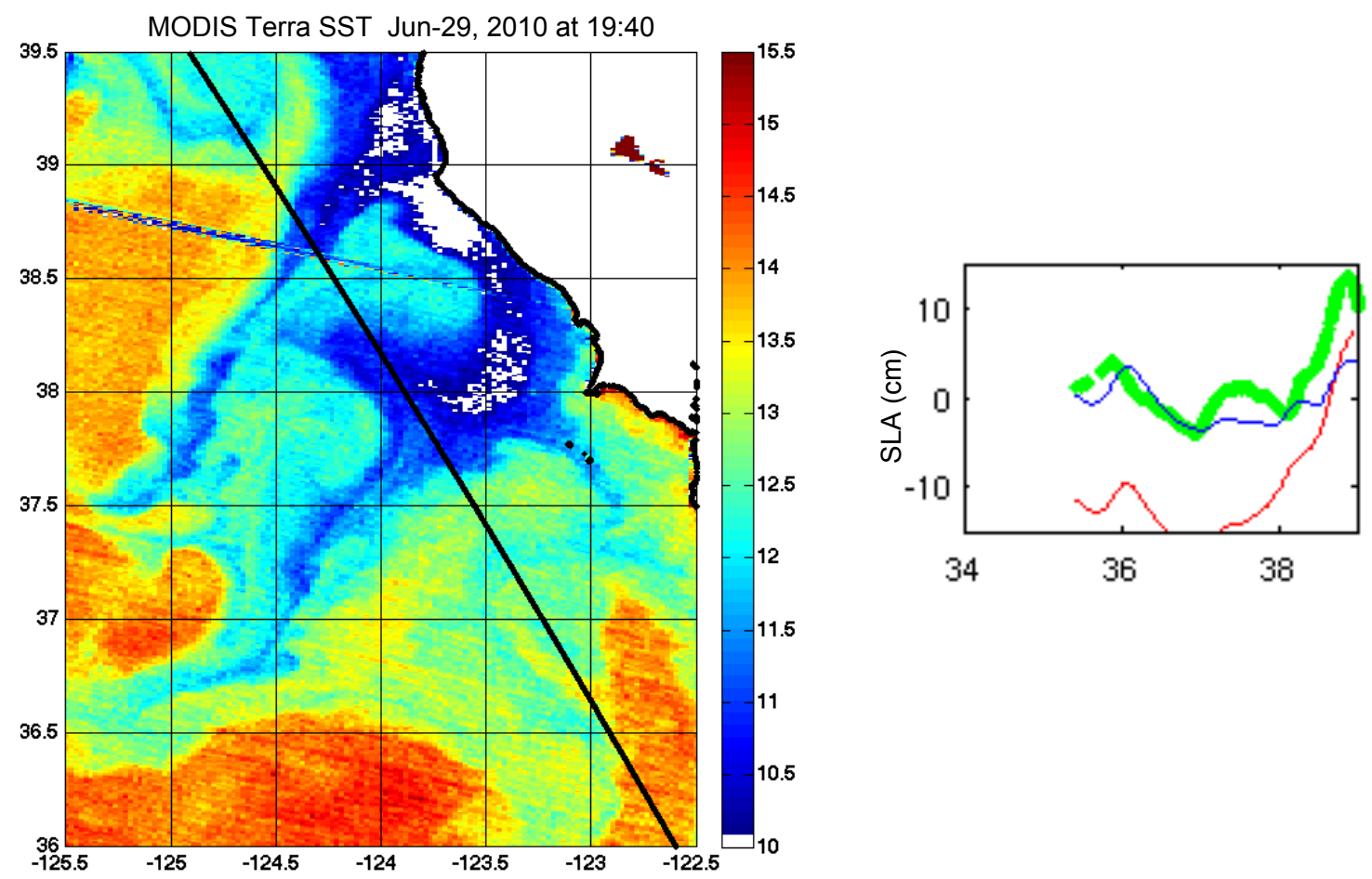

Figure 5.10: (Left) SST image from the MODIS sensor on Terra on Jun 29, 2010 at UTC 19:40, 18 hours after the altimeter time of passage (Right) Altimeter and HFR sea levels along P206 for C312 on June 29. In green: The Jason-1 SLA are smoothed with a 45-km low pass filter; In red: the raw HFR SLA; In blue: The OI Ekman Corrected HFR SLA

Using the Terra SST image from the MODIS satellite sensor on June 29, taken 18 hours after the satellite altimeter measurements, will help to debate whether the altimeter SLA over amplifies the signal or not (Fig. 5.10). The satellite SST only measures the sea surface temperature and may not reveal the dynamic height structure, unless the surface 
temperatures are representation of deep temperature features. This holds true for the cold upwelled filament, centered at $38.7 \mathrm{deg}$ and approximately $30 \mathrm{~km}$ wide, flowing across the altimeter track. This filament carries the signature of the upwelled water, while being in geostrophic adjustment associated with the meandering jet. The cold upwelling filament can

generate strong currents. The across track velocity observed by the altimeter is $\frac{\triangle S L A}{\Delta x} \frac{g}{f}=$ $\frac{8 \mathrm{~cm}}{25 \mathrm{~km}} \frac{9.81 \mathrm{~ms}^{-2}}{10^{-4} \mathrm{~s}^{-1}}=32 \mathrm{~cm} / \mathrm{s}$ which seems reasonable. The OI Ekman corrected HFR SLA measure a current half that velocity. Because the wind speeds are relatively high, around $39^{\circ}$ with strength exceeding $15 \mathrm{~cm} / \mathrm{s}$, there is a strong argument for the case of a misestimated Ekman correction. And because the winds at this location are strongest at the time of passage of the altimeter, shorter HFR currents averaging times do not resolve the problem, unlike the cases of C311 (June 19) and C319 (September 6). Finally this upwelling event is well captured for C314 (July 18) and C316 (August 7), when the winds are slower.

\subsection{HFR and Altimetry across track velocity anomaly comparison}

The cross-track geostrophic velocities from both the altimeter and HFR datasets are compared. The altimeter cross-track velocities are computed directly from the $20-\mathrm{Hz}$ data stream of sea level heights, $v_{a}=\frac{g}{f} \frac{d S L A}{d x}$, using finite difference, where $v_{a}$ is chosen to be positive if it contains an eastward component, and $d x$ is the distance between two $20-\mathrm{Hz}$ measurements which is around $300 \mathrm{~m}$. The finite difference operator acts as a high pass filter. To mitigate this noise, the resulting $20-\mathrm{Hz}$ velocities are filtered with a Lee filter over a window of 41 points $(\sim 12 \mathrm{~km})$. Finally two consecutive boxcar filter are applied over 91 points $(\sim 35 \mathrm{~km})$ and 151 points $(\sim 45 \mathrm{~km})$. For absolute height accuracy, path length and environmental correction (i.e. ionospheric, dry and wet tropospheric, tides, ect) are applied to the altimeter range measurements. These corrections are typically of large wavelength and it is unnecessary to include them for the calculation of the $20-\mathrm{Hz}$ velocities, where the distance between the measurements is around $300 \mathrm{~m}$. This is an advantage, especially in coastal regions when the inaccuracies in the corrections increase at the $1-\mathrm{Hz}$ level and can 
magnify the noise floor of the slope without improving the slope accuracy (Powell and Leben, $2004[64])$

Due to the noise content the altimeter geostrophic velocities require more filtering relative to the SLA to retrieve estimates consistent with the variability of the HFR crosstrack velocities.
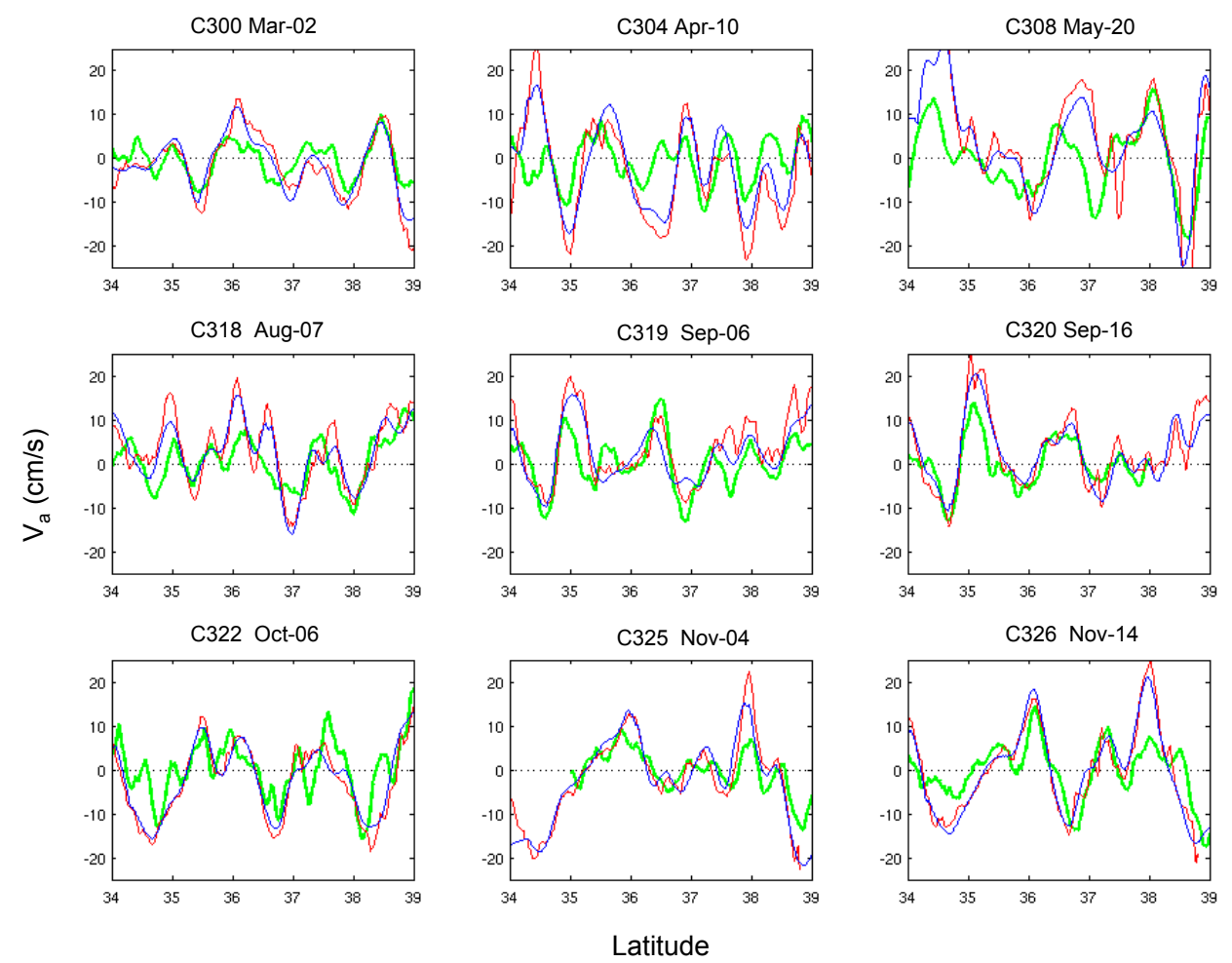

Figure 5.11: Cross track Geostrophic velocities for 9 cycles in 2010: Altimeter (in green), OI Ekman Corrected HFR (in blue), 3-day averaged Ekman Corrected HFR surface currents projected across track P206 (in red)

The cross-track HFR geostrophic velocities are computed in two ways. The first way is simply to project the 3-day averaged and Ekman corrected HFR currents across the P206 track. The other way is to calculate the cross velocities directly from the OI Ekman Corrected HFR SLA, using finite difference, without any additional filtering. As can be seen in Fig. 5.11, the two HFR cross-track velocities are very similar, which means that in regions when the OI cannot be implemented such as in nearshore coastal areas, or close to islands, 
the first method can still provide satisfying estimates of geostrophic velocities.

Another advantage of using the cross-track velocities is that differences between the altimeter and HFR measurements, that were observed as linear trends in the SLA, are now represented as local offsets, thus are less susceptible to create large RMS difference discrepancies over long distances. However, the velocity variations are noisier than for the SLA, and may be harder to interpret in terms of validating the altimeter measurements because it requires an additional differentiating step.

The correlations between the altimeter and HFR cross-track velocity anomalies are larger than 0.6 for 22 cases in 2010, with a mean correlation of $0.71 \pm 0.07$ (0.68 \pm 0.1$)$, and the RMS differences is $6.6 \pm 1.55 \mathrm{~cm} / \mathrm{s}(7.4 \pm 1.9 \mathrm{~cm} / \mathrm{s})$ using the $\mathrm{HFR}$ velocities computed from the OI Ekman Corrected SLA (3-day averaged Ekman Corrected currents). The crosstrack current anomalies STD were $14.3 \mathrm{~cm} / \mathrm{s}$ over the year 2010. The velocity RMS is within the limit of the geostrophic altimeter velocity error estimates found in the California Current System of 3-5 cm/s (Strub et al. 1997 [69], Powell and Leben 2004 [64]), and the velocity

resolution of $5 \mathrm{~cm} / \mathrm{s}$ from the HFR. Also the velocity RMS differences are consistent with the ones between current meters and altimetry observed to be $7-8 \mathrm{~cm} / \mathrm{s}$ in the CCS (Strub et al. 1997 [69]), and the ones between HFR and altimetry around $8 \mathrm{~cm} / \mathrm{s}$ for time series at specific locations along the Oregon Coastline (Saraceno et al. 2008 [67])

\section{$5.9 \quad$ Discussion-Overview}

In this section the HFR and altimeter SLA were compared and analyzed over a 750 $\mathrm{km}$ long offshore altimeter track. There are some cases that match well, but not all and the fundamental reasons are due to the wind history. There are also some limitations that arise from the smoothness of the HFR SLA product. There are some unresolved wind or wave induce surface drifts in the HFR current which are seen as linear drifts in the HFR SLA. These are hard to account for, especially in the presence of strong winds. The comparison of cross track geostrophic velocity anomalies reduces this problem, but it adds 
an additional noisy processing step of the altimeter sea levels measurements which we would like to validate. Taking into account the wind history can help decide whether the HFR geostrophic approximation will be adequate.

There are also other surface generated drifts, such as wave induced Stokes Drift. Stokes drift is a surface current produced by the waves propagating on the surface of the ocean. Its strength, depends linearly on the wind speed, instead of quadratically for the Ekman current. A wind speed of $10 \mathrm{~m} / \mathrm{s}$ can induce a $10 \mathrm{~cm} / \mathrm{s}$ Stokes Drift. As a test, the ECMWF Stokes Drift current produced every 6-hours on a $25 \mathrm{~km}$ resolution grid, were removed from the HFR currents, before the OI processing. The Stokes Drift and Ekman Corrected HFR SLA did not generate a better quality product. One reason is that the HFR surface current captures only a filtered version of this Stokes Drift, in a direction that may be distinct from the one on the surface.

In the next chapter, some Jason-2 ascending altimeter tracks are studied in a similar way near shore over a maximum along track of length of $150 \mathrm{~km}$. However in this section the HFR/Altimeter datasets were analyzed over a $750 \mathrm{~km}$ long track. To make the transition to the next chapter more meaningful, the statistics from this chapter are reevaluated. The RMS between the HFR/altimeter SLA are recomputed for 5 independent $150 \mathrm{~km}$ segments along P206, for each cycle. But before the RMS is computed, for each segment the HFR unknown offset is now being adjusted to the altimeter SLA on this $150 \mathrm{~km}$ long segment instead of the whole track. The overall HFR SLA will be discontinuous, but continuous by segments. This has the consequence to eliminate some of the linear drifts inconveniencies, and because this study is concerned with relative calibration of the altimeter data, and that both the HFR and altimeter spectrum indicates similarities above the $150 \mathrm{~km}$ wavelengths, this should not compromise the comparison too much. The mean of the RMS over the 36 cycles and over these 5 segments (a total of 180 segments) is $2.16 \pm 0.7 \mathrm{~cm}$, which is $30 \%$ less than the one calculated over the whole track. This segmentation may be a solution to the problem of the linear HFR SLA drifts, regarding the validation of the altimeter SLA. 


\section{Chapter 6}

\section{Methodology along altimeter tracks crossing the coastline}

\subsection{Introduction}

The Jason-1 and 2 ascending tracks moving from northwest to southeast, and travelling almost cross-shore to the California coastline are analyzed, especially the one passing over Conception Point, P221 travelling through Monterey Bay. The cross-track altimeter geostrophic velocities capture the variable alongshore flow. There are about $150 \mathrm{~km}$ of collocated altimeter and HFR data which is the furthest extent of the HFR surface currents. A novelty of this section is that the 2-km resolution HFR surface currents are available closer to shore, and the information gained by using them will be assessed on the validation of the altimeter data. Also several coastal retrackers included in the Jason-2 PISTACH product will be evaluated using the HFR SSH as the reference. Finally the altimeter is moving from the open ocean to the nearshore regions, and the space and time scales will be reduced. These changes need to be taken into consideration in the methodology to infer the SSH from the HFR surface currents. This section is a summary of the paper from Roesler et al. (2013 $[65])$.

\subsection{Motivation}

The region of interest will be mainly $25 \mathrm{~km}$ till about $150 \mathrm{~km}$ offshore and over the years 2008-2009 . Our domain does not include the near shore area. This was chosen to reduce the errors in range correction that accumulate in the SSH altimetric estimates as the altimeter 
approaches the coastal regions. The potential of HFR to correct coastal altimetric heights can still be explored, because there are unresolved issues in retrieving altimetry range in this domain such as rain events, low winds and small scales ocean dynamics. For example, Lee et al. (2010 [53]) find that, on average for Jason-2 from July 2008 until July 2009, a retracker developed for non-ocean surfaces improves the Brown-retracked SSH over the Californian continental shelf. The following questions will be addressed. How should we process the HFR surface current data to make them comparable with altimeter sea level measurements? How well are they related and what are the limitations of this comparison? Is there any information gained by validating the 2-km HFR data and high-frequency altimeter data? Can we use HFR to detect invalid segments of the traditional open-ocean retracked altimeter measurements and, if so, to decide which PISTACH retracker better fits the segment, as well as evaluate the performance of the retrackers under various sea state condition.

\subsection{Methodology}

Altimetry maps geostrophic flow only, whereas HFR data gives a total velocity and includes ageostrophic processes that will be partly eliminated using an Optimal Interpolation (OI) which forces the flow to be non-divergent. The spatial velocity covariances, determined from analysis of the HFR surface currents, diminish substantially with distance to shore (from $100 \mathrm{~km}$ to $10 \mathrm{~km}$ ) and the OI uses spatially varying spatial scales depending on the mapping grid point distance from the coast. The temporal covariance of HFR surface velocity is observed to decrease from 10-days in the open ocean to 3-days near the coast. Correspondingly, the input-currents to the OI, the first approximation of the geostrophic currents, at the time of passage of the altimeter, is selected as a 3-day average to remove the tides and short-term wind events. The velocity-noise error for the HFR is assumed to be constant $\mathrm{e}=15 \mathrm{~cm} / \mathrm{s}$. Note that in this section the Ekman component is not removed. The angle between these ground tracks and the North meridian at these latitudes is about $-30^{\circ}$ (symmetric position relative to the descending tracks). Consequently, the cross-track 
projection of the HFR surface currents should be less affected by the Ekman current contamination due to winds blowing predominantly in the north-south direction, in contrast to the descending track studied in the previous chapter. In fact, in this section the Ekman currents are not taken into consideration. Other reasons, are that the winds will not be well captured by the ECMWF gridded product, the Ekman coefficient may vary as we approach the coastline, and that the length of the along track studied (maximun of $150 \mathrm{~km}$ ) will not induce noticeable deviations in the HFR SLA, except maybe during high winds.

\subsection{Comparison over the open ocean with standard altimetry product}

The 6-km HFR heights were computed during 2008 along the altimeter track P221, which terminates in Monterey Bay, California (the nomination P221 is for Jason-1 prior to

its interleaved orbit and corresponds to Jason-2 P221). They were compared (first 12 cycles [65] Fig. 13) with the coincident along track Jason-1 standard open-ocean 1-Hz SLA product distributed by AVISO that were smoothed with both a $25 \mathrm{~km}$ cut-off and a $50 \mathrm{~km}$ cut-off in wavelength. From this small sample we can see that frequently both the HFR and altimetric heights agree well. In other cases the two sets diverge in segments that seem time dependent but not related to the distance from the coast. The correlation coefficients between the HFR SSHs and the 50-km filtered Jason-1 SSHs calculated for the 33 cycles during the year 2008 confirm our conclusions. There are a few sets that are significantly negatively correlated or not statistically different from zero, but the mean correlation, for 23 sets with a correlation larger than 0.5 , is 0.82 .

\subsection{Comparison with PISTACH retrackers}

The Jason-2 PISTACH coastal product provides 20-Hz range output for several retracking schemes: MLE-4: the 4 parameter Maximum Likelihood Estimator (the conventional Jason-2 deep-ocean retracker); Oce3: the 3 parameter MLE-3 performed on a de-noised waveform; Red3: MLE-3 done on a restricted analysis window around the leading edge, to 
remove the eventual gates corrupted by the effects of land; Ice3: a 30\% threshold method (Davis 1997 [23]), also implemented on a restricted analysis window. This will help to determine the feasibility of using the SSH computed from the HFR currents as a way to validate different retrackers. On a first experiment, the PISTACH retrackers were averaged to create a 1-Hz data stream along P221. They were processed without any editing (because examining the quality of the ocean retracked SSH and their potential improvement is one of the objectives of this study) and with the standard corrections applied, in a section of the ocean farther than $30 \mathrm{~km}$ offshore. Fig. 16 [65] illustrates the different behavior between ICE3 and MLE-4, that can be depicted. There are examples when both MLE-4 and Ice3 behave similarly, and either fit or not the HFR set. There are cases when either MLE-4 or Ice3 are

more consistent with the HFR data. Finally there are some sets when we could combine a segment of MLE-4 and a segment of Ice3 in order to get closer to the, presumed more accurate, HFR sea surface height estimates. Over the first 16 cycles during 2009, Ice3 is better correlated with HFR, except for 4 cases. When keeping only the highest correlated set, the mean correlation increase from 0.71 , if only MLE-4 were selected, to 0.82 . However the Threshold retracker uses an empirical relationship between the range and the shape of the waveform, it is not based on a physically sound model and care should be taken in its interpretation. This underlies the fact that even offshore the waveforms may diverge from the Brown model, and the conventional MLE retrackers do not apply.

\subsection{Explaining some disagreement between HFR and altimetry}

Various sea state conditions can affect the quality of altimeter SSHs. This can explain some of the disagreements between HFR SSHs and those from altimetry. For instance, the presence of unusually high Sig0 values ( $\operatorname{Sig} 0>16 \mathrm{~dB}$ for Jason-2) in the altimeter footprint, from blooming events, may signal a breakdown in the typical Brown model. The Blooming events, on P221 by Monterey Bay, extend over a few tens to hundreds of kilometers with unpredictable frequency. The Ice3 and Red3 retrackers were examined in relation to the sea 
state characterized by Sig0 and SWH. There is a tendency for Ice3 to fit better in cases of blooming events, and Red3 during high SWH. But this relation is not clear. In the future, this needs to be investigated more systematically to see if a trend could be predicted. Next the 20-Hz data stream are processed differently, to start examining the possibility of extracting a higher resolution product near shore and to compare it with the 2-km derived HFR SSH available over the year 2009. To edit the noisy $20-\mathrm{Hz}$ data a Lee filter is implemented and the smoothing scales are reduced to 7 and $21 \mathrm{~km}$. One source of caution: after the publication of the paper, it was discovered that the variability in the 2-km HFR SLA near the coast (for along track distance to shore $<50 \mathrm{~km}$ for P221, since the track travels through Monterey Bay) is an artifact of the OI methodology, due to the presence of coastal boundaries. So that for instance the near shore wiggles of the HFR SLA for C034 are noise. The 2-km HFR SSH field, derived from this methodology is slightly smoother than first expected. The consequence, is that it seems improbable to be able to use the HFR SLA to validate the altimeter data for spatial scales less than $20 \mathrm{~km}$.

Three-day-averaged HFR currents are used as input to the OI, but in the near-shore regions whether a 1 or 2-day average be more representative of the instantaneous altimeter along-track SSH, has not been tested. For the altimetry processing, the concerns are about editing (depending on the goals) and finding the best filtering to match the HFR heights variations.

This chapter presented the analysis of HFR and altimetry up to $25 \mathrm{~km}$ from the coast fro P221, and shows that in more than half the cases the HFR and altimeter match well (same conclusions for P145 to the north and P43 to the south). By referencing to the HFR SLA, several PISTACH retrackers are evaluated in relation to the sea state. The optimal method for altimetry retracking seems to be a combination of different retrackers for various segment of the altimeter track and depends on the cycle, even in the open ocean. However caution must be taken because the conditions for the consistency between HFR and altimetry are not well understood, except that it depends strongly on the wind history. 


\section{Chapter 7}

\section{Applications}

\subsection{Introduction}

Conventional open-ocean satellite altimetry is able to provide globally repeated sea surface information with very high accuracy resolution. However the retrieval of the sea level information is based on the assumption that the waveforms follow the typical ocean Brown waveform model. When this is not the case, other retracking procedures need to be implemented, or the data is flagged as bad. The previous analyses have demonstrated that under certain conditions the geostrophic HFR field matches the one from altimetry. Consequently these can be used as a validation reference for the retracking method along an altimeter track, when the waveforms do not conform to the Brown model.

\subsection{Open Ocean}

In the open ocean, in cases of blooming events, such as low winds or rain cells, the waveforms will be contaminated. These cases are easily detectable, by the rain flag, or auxiliary wind information. Fig. 7.1 provides an example of a rain event, before latitude $36^{\circ}$ N. Furthermore, in the open ocean, some of the environmental corrections that need to be applied to the altimeter SSH will not be affected by the event. The effects of these events on the waveform will depend on the extent of the problem. Some will be close to the Brown model, others will have a very different shape. For the latter new retracking methods may have to be developed which will enhance the retracking theory. In instances of low winds 
or surface slicks, the ocean surface backscatter along the altimeter track can be given by inversion of the altimeter waveforms (Tournadre et al. 2011 [72]). This information can in turn be used to retrack the waveforms. Having the HFR SLA data as a validation reference along an altimeter track, instead of a point measurement or the geoid is an improvement.

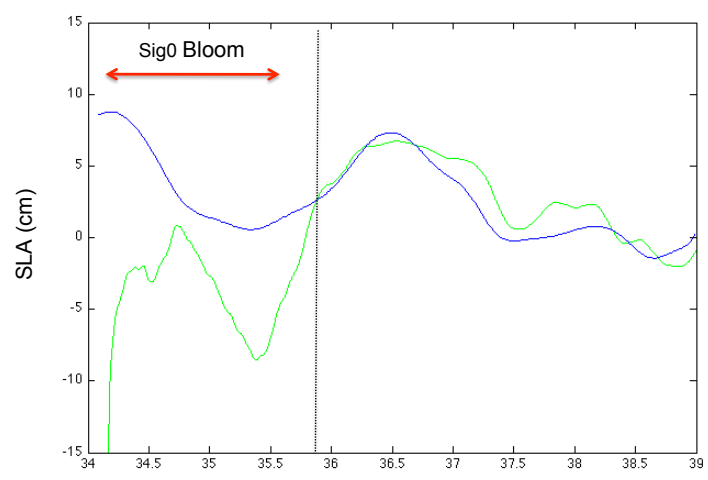

Figure 7.1: Jason-1 C323 Sep 6: HFR ( blue) and altimetry (green) SLA. There is a rain event before latitude $36^{\circ} \mathrm{N}$

Yet one needs to recall that the HFR surface currents are less reliable in the event of high SWH (strong storms) and may be unavailable during low winds. However these low wind events have been noted to last only a few hours, and doing a three day averaging on the HFR currents alleviates the problem.

\subsection{Near shore: case study Jason-2 P206 in the California Bight}

In this section the near-shore section of the descending Jason-2 P206 passing through the California Bight (Fig. 7.2) is studied. The altimeter track leaves the California coast, travels between the two islands of Santa Rosa and Santa Cruz located $50 \mathrm{~km}$ off the main coast, and then $80 \mathrm{~km}$ later passes over St Nicholas Island. The 2-km HFR data extends almost up to St Nicholas Island. The two islands Santa Rosa and Santa Cruz are separated by about $12 \mathrm{~km}$, and P206 is at its closest approach about $2 \mathrm{~km}$ away from Santa Rosa. At a distance of about $10 \mathrm{~km}$ away from land, the waveforms will be contaminated, but the 
Jason-2 tracker does not lose track, so the altimeter data is available throughout the whole region.
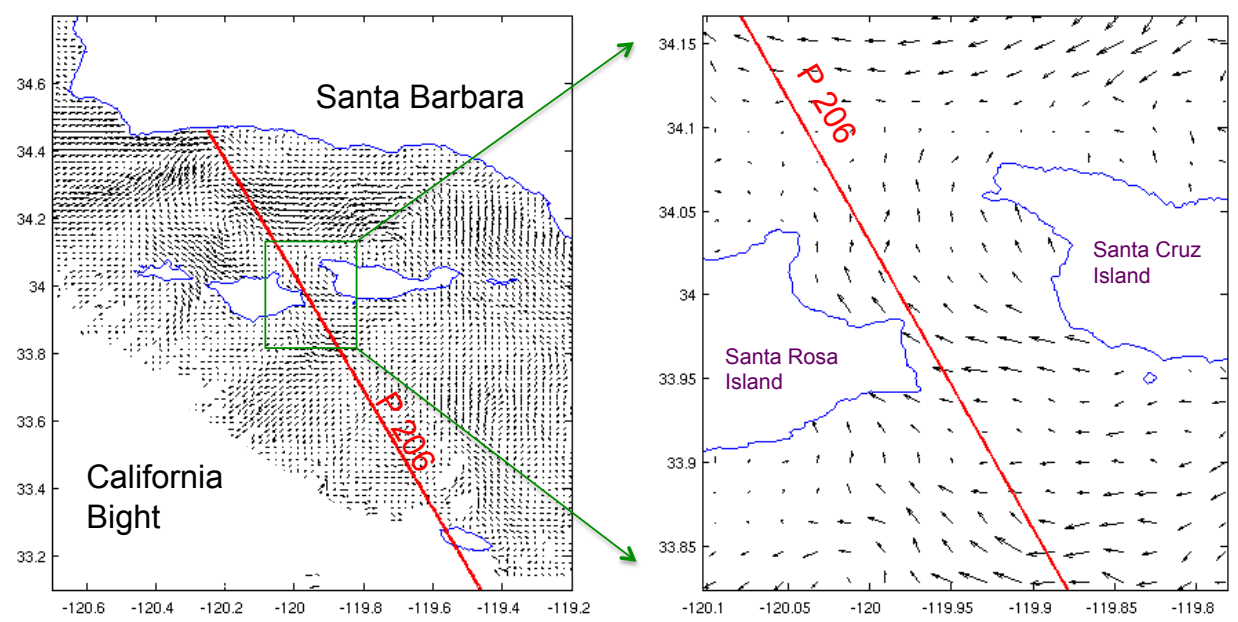

Figure 7.2: (Left) 2-km HFR ocean surface currents (in black) in the California Bight. Jason-2 P206 in red). (Right) Close up, between the two islands of Santa Rosa and Santa Cruz.

In this study over the year 2010, the HFR ocean currents are averaged over 2 days, and then are projected cross track. Finally the HFR SSH are computed by integrating the HFR cross track velocities along P206, and interpolated to the same location as the altimeter data.

The entire 20-Hz range MLE-4 data are kept, without any editing, because the behavior of the MLE-4 retracker is analyzed. All the corrections are applied to obtain the SLA, except that the Composite Water Vapor correction is used (to avoid the altimeter radiometer water vapor correction contamination by the land), the GIM ionospheric correction is used and that the SSB is not applied (the SSB is computed from the sig0 and SWH retrieved by waveform retracking, and will be erroneous, when the waveforms are contaminated by land)

The mean of the SLA data over the year 2010 (36 cycles) (Fig. 7.3) displays the typical behavior pattern of the MLE-4 retracker when it passes over land. There are clear overshoots of the mean SLA over land, although not as much as it travels between the 2 islands. 
a)
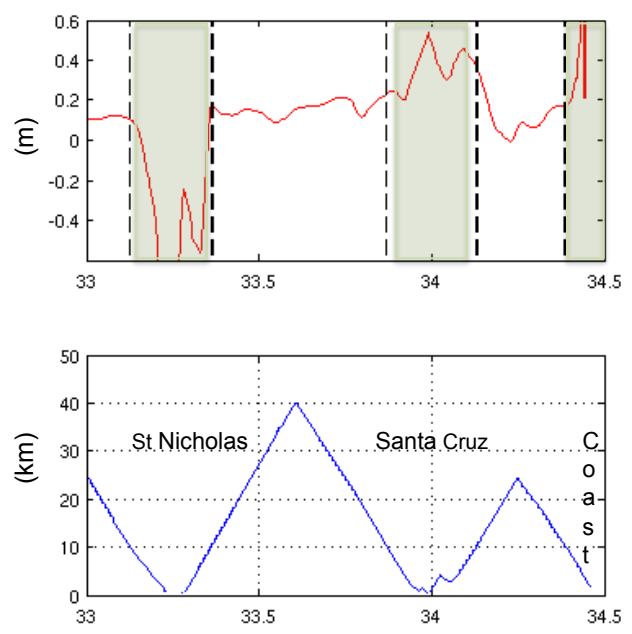

b)

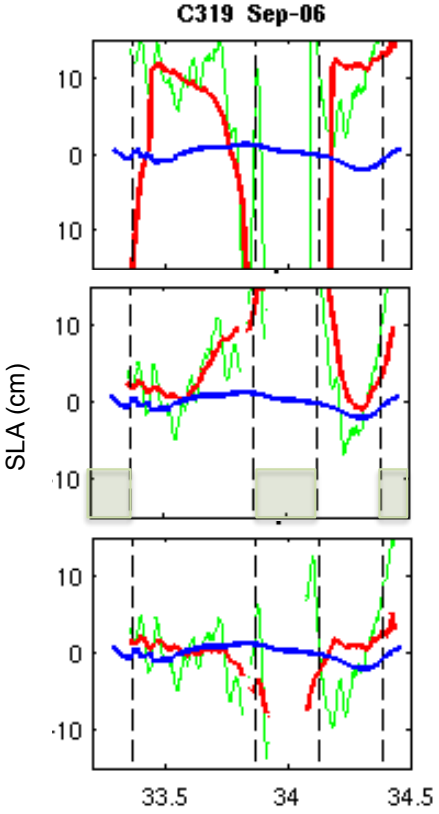

Figure 7.3: (Left) Top: the 2010 mean of the altimeter MLE-4 SLA. Bottom: the P206 distance from a coastal feature. The coast is on the right side of the figure. (Right) Comparing altimeter and HFR SLA along P206. In blue: HFR SLA. In red: altimeter SLA after a boxcar filter of 91 points. In green: altimeter SLA after a boxcar filter of 21 points. The black dotted lines delineate the 10-km borders of the coastal features. The main coast is on the right side of the figures. (top) All the altimeter data is kept and the 2010 SLA mean is not removed. (middle) All the altimeter data is kept but the 2010 SLA mean is removed. (bottom) The extreme outliers are removed, and the 2010 SLA mean is removed

The mean of the HFR data over the year 2010 is removed to access the data SLA. But the question is, can the 2010 SLA mean, which is erroneous over land, be removed from the altimeter SLA or not. Fig. 7.3 (right top) display the altimeter SLA before removing the 2010 mean, and (right middle) after. It demonstrates that using this mean is valuable, because it contains the typical pattern of the MLE- 4 retracker on the SSH, which is then reduced by applying the mean. Furthermore, if the extreme outliers are removed, and then the mean applied Fig. 7.3 (right bottom) the altimeter SLA becomes closely related to the HFR SLA. This implies that even though the altimeter data are contaminated in several sections, they can still provide a clue to know whether the corresponding HFR SLA set could be used as a validation tool. 

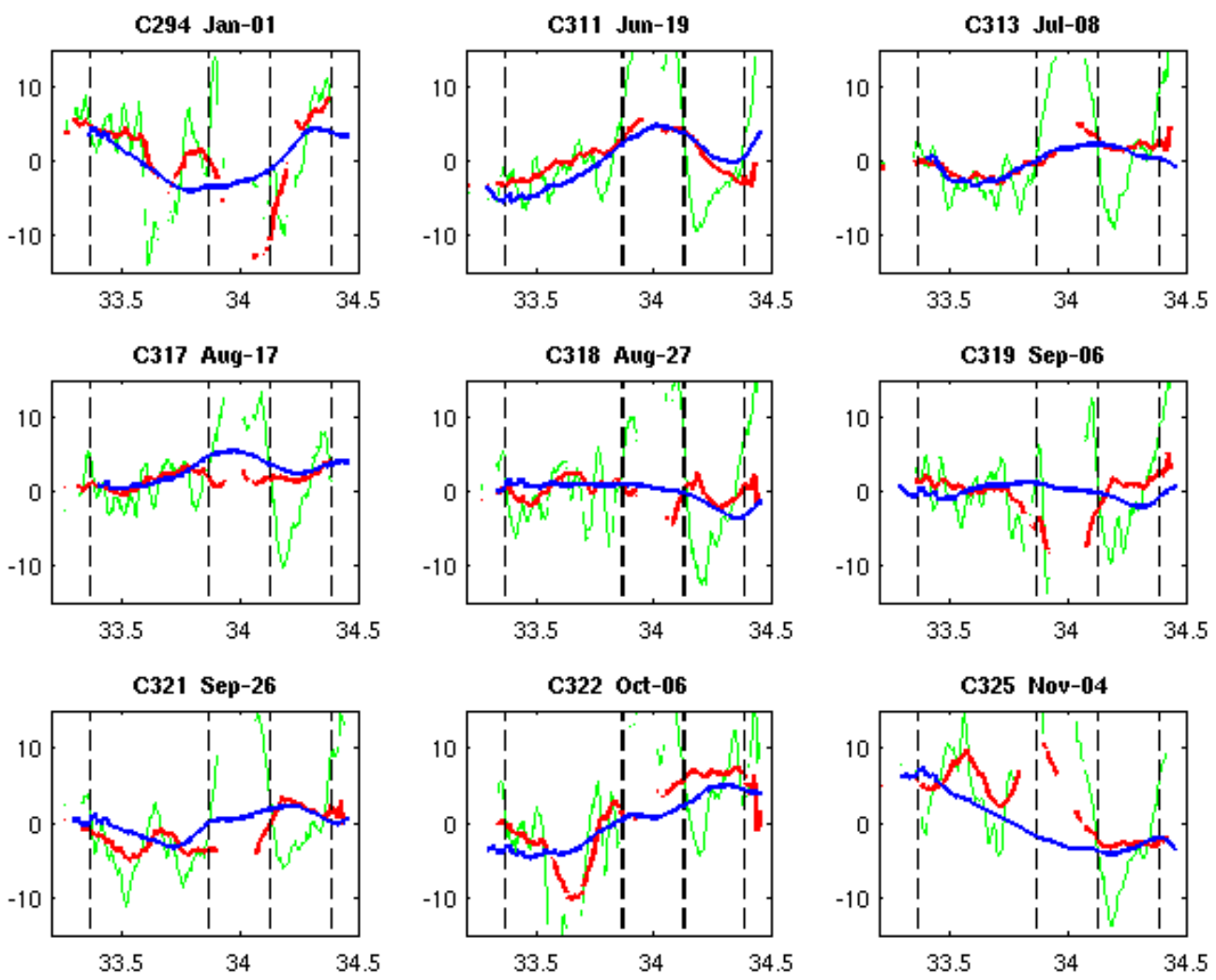

Figure 7.4: Comparing altimeter and HFR SLA along P206. In blue: HFR SLA. In red: altimeter SLA after a boxcar filter of 91 points. In green: altimeter SLA after a boxcar filter of 21 points. The black dotted lines delineate the $10-\mathrm{km}$ borders of the coastal features. The main coast is on the right side of the figures.

Fig. 7.4 presents 9 cycles when the match is satisfying. Several retracking systems can then be tested, such as the Brown Model with a Gaussian/Asymmetric Gaussian peak retracker (Halimi et al. 2012 [37]) and validated using the HFR SLA. Note that on these figures some erroneous altimeter SLA segments are not connected to land contamination, such as for C294 and C322 around 33.5 . 


\section{Chapter 8}

\section{Conclusions}

One of the challenges of using satellite altimetry in the coastal ocean is correcting for distortions of the altimetric waveforms linked to the presence of possible rapid changes in sea states and/or the presence of land within the altimeter footprint. Many retracking procedures have been developed but there is great difficulty in knowing what is the proper method and where it is best applied. The goal of this research was to evaluate the skill of the HFR coastal surface currents to validate the retrackers, which would be a marked improvement compared to sparse in-situ point validation measurements. The U.S. West coast HFR network monitors hourly ocean surface currents with an offshore range up to $150 \mathrm{~km}$ and spatial resolutions of 2 and $6 \mathrm{~km}$ depending on the radar operating frequency. The California Current System is a dynamic region driven by strong wind forcing. Coastal upwelling along coastline with large promontories and over strongly sloping bottom topography generates a rich eddy field. This makes the California coastal region a perfect laboratory to analyze the relationship between the altimeter SSH measurements and the HFR ocean surface currents.

Altimetry maps geostrophic flow only, whereas HFR data gives a total surface velocity averaged over the top meter of the ocean and includes ageostrophic processes that need to be eliminated. In particular the HFR contain surface wind drift components, including the Ekman current. Using the AVISO gridded altimeter geostrophic absolute velocities and the ECMWF gridded winds, the Ekman coefficients are estimated for the HFR surface currents using a simple linear model, in the open ocean. The Ekman corrected HFR surface 
currents are then averaged over time to remove the tides and the inertial currents. Finally by analyzing the time and space scales of the coastal oceanic features, a stream function is fitted to the HFR coastal currents to retrieve their matching SSHs, which are mapped with varying spatial scales using optimal interpolation. At the large mesoscales $(100 \mathrm{~km}$ and 7 days), the geostrophic HFR and gridded altimeter datasets are in very good agreement with correlations larger than 0.8 over a 3 year period. The RMS of the differences is $1.8 \mathrm{~cm}$ in SLA and $4.5 \mathrm{~cm}$ in velocity anomalies for both the zonal and meridional components.

Analyzing a $750 \mathrm{~km}$ long offshore altimeter track parallel to the coastline further refines the relationship. Over the year 2010, 60\% of the cases have a correlation larger than 0.6 and a resulting RMS of the differences of $2.5 \mathrm{~cm}(6.5 \mathrm{~cm} / \mathrm{s})$ in SLA (across-track geostrophic velocity anomaly), while the STD of the altimeter signal is $4.5 \mathrm{~cm}(14.3 \mathrm{~cm} / \mathrm{s})$. One drawback is that the HFR SLA, from this methodology, are relatively smooth, and do not contain much signal below the $50 \mathrm{~km}$ wavelengths. Reducing the time averaging from 3 to 1 day does not produce more information. This imposes a limit on the along track length scales that can be used for the validation of the altimeter measurements using the HFR data as a reference The altimeter data contain some instrumental white noise, which affects the altimeter signal for wavelengths less than $30 \mathrm{~km}$, part of it is of dynamical content, but separating the signal from the noise is a delicate issue.

It is shown that the match between the HFR/altimeter SLA depends on the wind history, essentially because a residual Ekman current in the HFR, will create a linear shift in the HFR SLA. The use of the cross-track geostrophic velocity anomalies alleviates this problem, but it also means that there is one more additional step in the processing of the altimeter data, which is a differentiator.

The analysis is then extended to ascending Jason-1 and 2 altimeter tracks that cross the coastline. As the altimeter travels from the one ocean to the coast, the oceanic space and time scales decrease. The 2-km HFR surface currents become available. Along these $150 \mathrm{~km}$ long collocated HFR and altimetry segments, the Ekman linear drift effect will be 
less problematic, and the Ekman current is not removed prior to the OI. The statistics for the regions $25-150 \mathrm{~km}$, not affected by land contamination, are slightly better than in the previous case. The variability of the $2-\mathrm{km}$ HFR SLA data are still relatively smooth. So the possibility to validate small scales coastal dynamics from the altimeter retrievals seems limited. But perhaps the small scales currents are weak, and the HFR SLA do represent the dynamics heights.

By referencing to the HFR SLA, several PISTACH retrackers are evaluated in relation to the sea state. The optimal method for altimetry retracking seems to be a combination of different retrackers for various segment of the altimeter track and depends on the cycle, even in the open ocean. However caution must be taken, because even though some matches are almost perfect, the conditions for the consistency between HFR and altimetry are not well understood, except that it depends strongly on the wind history.

Some of the errors between the altimeter and HFR SLA come from the measurements errors of each remote sensing instrument; others from the time sampling difference: one is instantaneous, the other an hourly-average which is then further averaged over time; others derive from the fact that the instrument do not measure the same ocean parameter; finally there are the processing errors to make the measurements comparable. So some phase shifts between the SLAs seems unavoidable, the linear drift is explained by the wind and wave induced surface drifts. But there are examples when the altimeter depicts a strong signal that seems undetected by the HFR surface currents. The question remains whether those are true ocean dynamic signals not captured by the HFR from our processing method, or if there are some specific ocean dynamics that affect slightly the altimeter waveform.

The new applications concern the validation of the retracking techniques along an altimeter track and will provide a continuity between the open ocean and coastal retracked SSH. This would be a great improvement compared to sparse point measurements validation methods. The inferred geostrophic HFR field can be used as a validating reference, as long as there are sufficient collocated valid points on the altimeter track, so that the quality of 
the HFR geostrophic data can be estimated. In the open ocean specific retrackers could be implemented for cases of blooming events. Near shore, the California Bight example provides a very promising location to further develop this methodology and test several dedicated coastal retrackers. The experience gained from validating the coastal retrackers when HFR is available, could then be transferred to other remote coastal regions. 


\section{Bibliography}

[1] L. Amarouche, P. Thibaut, O. Zanife, J-P. Dumont, P. Vincent, and N. Stenou. Improving the Jason-1 ground retracking to better account for attitude effects. Marine Geodesy, (1-2):171-197, 2004.

[2] O.B. Andersen and R. Scharroo. Range and geophysical corrections in coastal regions: And implications for mean sea surface determination. In Vignudelli S., A.Kostianoy, P. Cipollini, and J.Benveniste, editors, Coastal Altimetry, pages 103-145, Berlin, 2011. Springer.

[3] F. Ardhuin, L. Marie, N. Rascle, P. Forget, and A. Roland. Observation and estimation of Lagrangian, Stokes, and Eulerian currents induced by winds and waves at the sea surface. J. of phys oceanography, 39:2820-2838, 2009.

[4] AVISO/Altimetry. Coastal and Hydrology Altimetry Product (PISTACH) Handbook, CLS-DOS-NT-10-246. CNES/CLS, France, salp-mu-p-op-16031-cn 01/00, ed.1.0, "http://www.aviso.oceanobs.com/fileadmin/documents/data/tools/hdbk_pistach.pdf" edition, 2010.

[5] AVISO/Altimetry. SSALTO/DUACS Users Handbook DT CorSSH and DT SLA Product. CNES/CLS, France, cls-dos-nt-086.381, ed. 2.0. "http://www.aviso.oceanobs.com/fileadmin/documents/data/tools/ hdbk_dt_corssh_dt_sla.pdf" edition, 2012.

[6] AVISO/Altimetry. SSALTO/DUACS Users Handbook MSLA and MADT Near-Real Time and Delayed Time Products CLS-DOS-NT06.034. CNES/CLS, France, salp-mu-p-ea-21065-cls, ed.3.4,

"http://www.aviso.oceanobs.com/fileadmin/documents/data/tools/hdbk_duacs.pdf" edition, 2013.

[7] D. E. Barrick, M. W. Evans, and B. L. Weber. Ocean surface currents mapped by radar. Science, 198:138-144, 1977.

[8] D. E. Barrick and B. J. Lipa. Evolution of bearing determination in HF current mapping radars. Oceanography, 10(2):72-75, 1997. 
[9] D. E. Barrick and B. J. Lipa. Radar angle determination with MUSIC direction finding. (U.S. Patent):5 990 834, 1999.

[10] J. Barth and B. M. Hickey. West coast oceanography: Implications for ballast water exchange. Pacific States Marine Fisheries Commission, 2005.

[11] F. Birol, M. Cancet, and C. Estournel. Aspects of the seasonal variability of the northern current (NW Mediterranean Sea) observed by altimetry. J. Mar. Syst., 81:297-311, 2010.

[12] J. Bouffard, Vignudelli S., Hermann M., Lyard F., P. Marsaleix, Y. Ménard, and P. Cipollini. Comparison of ocean dynamics with a regional circulation model and improved altimetry in the North-western Mediterranean. Terr. Atmos. Ocean. Sci., 19:117-133, 2008.

[13] F. D. Bretherton, R. E. Davis, and C. B. Fandry. A technique for objectve analysis and design of oceanographic experiments applied to MODE-73. Deep Sea Res., pages 559-582, 1976.

[14] G. Brown. The average impulse response of a rough surface and its applications. IEEE Trans. Antennas Propag., 25:67-74, 1977.

[15] S. Brown. A novel near-land radiometer wet path-delay retrieval algorithm: Application to the Jason-2/OSTM advanced microwave radiometer. IEEE Trans. Geosci. Remote Sens, 48(4):1986-1992, 2010.

[16] X. Capet, J. C. McWilliams, M.J. Molemaker, and A.F. Shchepetkin. Mesocale to submesocale transition in the California Current System part II: Frontal processes, eddy flux, and obsevational tests. Journal of Phys. Ocean., 38(1):29-43, 2008.

[17] P. C. Chavanne and P. Klein. Can oceanic submesoscale processes be observed with satellite altimetry? Geophys. Res. Lett., 37:L22602, 2010.

[18] D. M. Checkley and J. A. Barth. Patterns and processes in the California Current System. Progress In Oceanography, 83:49-63, 2009.

[19] D. Chelton, J. Ries, B. Haines, L. Fu, and P. Callahan. Satellite altimetry. In L. Fu and A. Cazenave, editors, Satellite Altimetry and Earth Sciences: A Handbook of Techniques and Applications, pages 1-132, New York, 2001. Elsevier.

[20] D. Chelton, M. G. Schlax, R. M. Samelson, and R. A. de Szoeke. Global observations of large oceanic eddies. Geophys. Res. Lett., 34:L15606, 2007.

[21] P. Cipollini, S. Vignudelli, F. Lyard, and L. Roblou. 15 years of altimetry at various scales over the mediterranean. In V. Barale and M. Gade, editors, Remote Sensing of European Seas, pages 296-306, Heidelberg, Germany,, 2008. Springer.

[22] D. D. Crombie. Doppler spectrum of sea echo at $13.56 \mathrm{Mc} / \mathrm{s}$. Nature, 175:681-682, 1955. 
[23] C. H. Davis. A robust threshold retracking algorithm for measuring ice-sheet surface elevation change from satellite radar altimetry. IEEE Trans. Geosci. Remote Sens., 35(4):974-979, 1997.

[24] X. Deng. Improvement of geodetic parameter estimation in coastal regions from satellite radar altimetry. PhD thesis, Curtin Univ. of Technol, Perth, Australia, 2004.

[25] X. Deng and W. E. Featherstone. A coastal retracking system for satellite radar altimeter waveforms: Application to ERS-2 around Australia. J. Geophys. Res., 111:C06012, 2006.

[26] X. Deng, W. E. Featherstone, C. Hwang, and P. A. M. Berry. Estimation of contaminations of ERS-2 and POSEIDON satellite radar altimetry close to the coasts of Australia. Mar. Geod., 25(4):249-271., 2002.

[27] X. Deng, W. E. Featherstone, C. Hwang, and C. Shum. Improved coastal marine gravity anomalies at the Taiwan Strait from altimeter waveform retracking. In C. Hwang, C. Shum, and J. Li, editors, International Association of Geodesy Symposia, volume 126, pages 41-45, Berlin, Heidelberg, 2003. Springer.

[28] C. Desportes, E. Obligis, and L. Eymard. On the wet tropospheric correction for altimetry in coastal regions. IEEE Trans. Geosci. Remote Sens., 45(7):2139-2149, 2007.

[29] G. Egbert, A. Bennett, and M. Foreman. TOPEX/Poseidon tides estimated using a global inverse model. J. of Geophys. Res., 99(C12):24,821 - 24,852, 1994.

[30] G. Egbert and S. Erofeeva. Efficient inverse modeling of barotropic ocean tides. J. Atmos. Oceanic Technol., 19(2), Feb 2002.

[31] V. W. Ekman. On the influence of the Earth's rotation on ocean currents. Ark. Mat. Astron. Fys., 2:1-53, 1905.

[32] Stokes G.G. On the theory of oscillatory waves. Trans, Cambridge Phil. SOc., 8:441-455.

[33] J. Gómez-Enri, I. Caballero, G. Navarro, and P. Villares. Towards a validation of ENVISAT RA-2 high rate significant wave height in coastal systems: Case study of the Gulf of Cadiz,. Prague, Czech Republic, June 2011. 2011. 5th EARSeL Workshop on Remote Sensing of the Coastal Zone.

[34] C. Gommenginger, P. Thibaut, L. Fenoglio-Marc, X. Deng, J. Gómez-Enri, and Y. Gao. In Coastal Altimetry, chapter Retracking altimeter waveforms near the coasts,, pages 61-10. Springer, Berlin, 2011.

[35] Hayne G.S. Radar altimeter mean retrun waveform from near-normal incidence ocean surface scattering. IEEE Trans. Antennas Propag., 28(5):687-692, 1980.

[36] K.W. Gurgel, A. Dzvonkovskaya, A. Pohlmann, T. Schlick, and E. Gill. Simulation and detection of tsunami signatures in ocean surface currents measured by HF radar. Ocean Dyn., 2008. 
[37] A. Halimi, C. Mailhes, J-Y Tourneret, P. Thibaut, and F. Boy. Parameter estimation for peaky altimetric waveforms. Trans. Geosc. and Remote sens., 2012.

[38] G. Herbert, N. Ayoub, P. Marsaleix, and F. Lyard. Signature of the coastal circulation variability in altimetric data in the Southern Bay of Biscay during winter and fall 2004. J. Mar. Sys., 88(2):139-158, 2011.

[39] B. M. Hickey. Coastal oceanography of Western North America from the tip of Baja California to Vancouver Island. The Sea, 11:345-393, 1998.

[40] C. Hwang and S. A. Chen. Circulation eddies over the South China sea derived from Topex/Poseidon altimetry. J. Geophys. Res., 105(23):943-965, 2000.

[41] C. Hwang, J. Guo, X. Deng, H. Y. Hsu, and Y. Liu. Coastal gravity anomaly from retracked Geosat/GM altimetry: Improvement, limitation and role of airborne gravity data,. J. Geod., 80:204-216, 2006.

[42] Kundu P. K. Ekman veering observed near the ocean bottom. J. of Phys oceanography, 6(2):238-242, 1976.

[43] Matthews D. K. Examinig the Wind forced velocity structure of the California Current System using Observations derived from Satellite remote sensing. PhD thesis, Uni. of Colorado at Boulder, 2008.

[44] K. A. Kelly, R. C. Beardsley, R. Limeburner, K.H. Brink, J.D. Paduan, and T.K. Chereskin. Variabilty of the near-surface eddy kinetic energy in the California Current based on altimeter, drifter and moored current meter data. J. Geophys. Res., 103:1306713083, 1998.

[45] S. Y. Kim. Observations of submesoscale eddies using high-frequency radar-derived kinematic and dynamic quantities. Cont. Shelf Res., 30:1639-1655, 2010.

[46] S. Y. Kim, E. J. Terrill, and B. D. Cornuelle. Mapping surface currents from HF radar radial velocity measurements using optimal interpolation. J. Geophys. Res., 113:C10023, 2008.

[47] S. Y. Kim, E. J. Terrill, B. D. Cornuelle, B. Jones, L. Washburn, M. A. Moline, J. D. Paduan, N. Garfield, J. L. Largier, G. Crawford, and P. M. Kosro. Mapping the U.S West Coast surface circulation: A multiyear analysis of high-frequency radar observations. J. Geophys. Res., 116:C03011, 2011.

[48] J. T. Kirby and Tsung-Muh Chen. Surface waves on vertically sheared flows: Approximate dispersion relations. J Geophys Res, 94(C1):1013-1027, 1989.

[49] K. E. Laws and J. D. Paduan. Error assessment of HF radar based ocean current measurements: An error model based on sub-period measurement variance,. Monterey, Calif, 2011. IEEE/OES Current, Waves and Turbulence Measurement Conference. 
[50] M. Le Hénaff, L. Roblou, and J. Bouffard. Characterizing the Navidad current interannual variability using coastal altimetry. Ocean Dyn., 61:425-437, 2010.

[51] P.-Y. Le Traon, F. Nadal, and N. Ducet. An improved mapping method of multisatellite altimeter data. J. Atmos. Oceanic Technol., 15:522-534, 1998.

[52] N. A. Lebedev, A. G. Kostianoy, A. I. Ginzburg, D. P. Medvenev, N. A Sheremet, and S. N. Shauro. In Coastal Altimetry, chapter Satellite altimetry applications in the Barents and White Sea, pages 389-415. Springer, Berlin, 2011.

[53] H. Lee, C. Shum, W. Emery, X. Deng, C. Roesler, Y. Yi, C. Kuo, Z. Lu, and S. Calmant. Validation of Jason-2 altimeter data by waveform retracking over California coastal ocean. Mar. Geod., 33(1):304-316, 2010.

[54] H. Lee, C. Shum, Y. Yi, M. Ibaraki, J. Kim, A. Braun, C. Kuo, and Z. Lu. Louisiana wetland and water level monitoring using retracked TOPEX/POSEIDON altimetry. Mar. Geod., 32:284-302, 2009.

[55] J. S. Lee. Digital image enhancement and noise filtering by use of local statistics. IEEE Transactions on Pattern Analysis and Machine Intelligence, PAMI-2:165-168, 1980.

[56] E. W. Leuliette and L. Miller. Closing the sea level rise budget with altimetry, Argo, and GRACE. Geophys. Res. Lett., 36:L04608, 2009.

[57] B. J. Lipa and D. E. Barrick. Extraction of sea state from HF radar sea echo: Mathematical theory and modeling. Radio Sci., 21(1):81-100, 1986.

[58] Y. Liu, R.H. Weisberg, S. Vignudelli, L. Roblou, and C.R.Merz. Comparison of the XTRACK altimetry estimated currents with moored ADCP and HF radar observations on the WestFlorida Shelf. Adv. Space Res., 50(8):1085-1098, 2012.

[59] P. Marchesiollo, J. C. McWiliams, and A. F. Shchepetkin. Equilibrium structure and dynamics of the california current system. J. Geophys. Res, 33:753-783, 2003.

[60] G. Mitchum, D. Hancock, G. Hayne, and D. Vandemark. Blooms of Sig0 in the Topex radar altimeter data. J. Atmos. Oceanic Technol., 21:1232-1245, 2004.

[61] R. Morrow and P. Y. Le Traon. 15 years of satellite altimetry and mesoscale ocean dynamics observed with 15 years of altimetric data. Venice, Italy, 2006. Symposium on 15 Years of Progress in Radar Altimetry, ESA.

[62] Rascle N. Impact of waves on the ocean circulation. PhD thesis, Uni. de Bretagne Occidentale (France), 2007.

[63] J. D. Paduan and L. K. Rosenfeld. Remotely sensed surface currents in Monterey Bay from shore based HF radar (Coastal Ocean Dynamics Application Radar). J Geophys Res, 101(C9):20669-20686, 1996. 
[64] B. S. Powell and R. R. Leben. Filter for geostrophic mesoscale currents from along-track satellite altimetry. J. Atmos. Oceanic Technol., 21:1633-1642, 2004.

[65] C. J. Roesler, W. J. Emery, and S.Y. Kim. Evaluating the use of high-frequency radar coastal currents to correct satellite altimetry. J Geophys Res, 118(C):1-20, 2013.

[66] S. Ruiz, A. Pascual, B. Garau, I. Pujol, and J. Tintorè. Vertical motion in the upper ocean from glider and altimetry data. Geophys. Res. Lett., 26:L14607, 2009.

[67] M. Saraceno, P. T. Strub, and P. M. Korso. Estimates of sea surface height and nearsurface alongshore coastal currents from combinations of altimeters and tide gauges. J. Geophys. Res., 113:C11013, 2008.

[68] J. Severini. Estimation et classification de Signaux Altimètriques. PhD thesis, IPN, Toulouse, France, 2010.

[69] P. T. Strub, T.K. Chereskin, P. Niiler, C. James, and M.D. Levine. Altimeter-derived variablity of surface velocities in the Calilfornia Current System. 1 Evaluation of TOPEX altimeter velocity resolution. J. of Geophys. Res., 6:C12727-12748, 1997.

[70] P.T. Strub and C. James. Altimeter-derived surface circulation in the NE Pacific Gyres: Part I Seasonal variability. Progress In Oceanography, (53):163-183.

[71] J. Tournadre. Validation of Jason and Envisat altimeter dual frequency rain flags. Marine Geodesy, 27:153-169, 2004.

[72] J. Tournadre, B. Chapron, and N. Reul. High-resolution imaging of the ocean surface backscatter by inversion of altimeter waveforms. J. Atmos. Oceanic Technol., 28(8):1050-1062, 2011.

[73] J. Tournadre, B. Chapron, N. Reul, and D. C. Vandemark. A satellite altimeter model for ocean slick detection. J. Geophys. Res, 111:C04004, 2006.

[74] N. Tran, S. Labroue, S. Phillips, E. Bronner, and N. Picot. Overview and update of the sea state bias corrections for the Jason-2, Jason-1 and TOPEX missions. Mar. Geod., 33(S1):348-362, 2010.

[75] J. L. Wilkin, M. S. Bowen, and W. J. Emery. Mapping mesoscale currents by optimal interpolation of satellite radiometer and altimeter data. Ocean Dyn., 52(3):95-103, 2002. 
Appendix A

JGR paper Roesler et al. 2013 


\title{
Evaluating the use of high-frequency radar coastal currents to correct satellite altimetry
}

\author{
C. J. Roesler, ${ }^{1}$ W. J. Emery, ${ }^{1}$ and S. Y. Kim ${ }^{2}$ \\ Received 22 August 2012; revised 25 April 2013; accepted 26 April 2013; published 1 July 2013.
}

[1] Coastal altimeter waveforms may differ from the ones in the open ocean, either from rapid changes in the sea state or the presence of land within the satellite altimeter footprint. The optimal retracking method for an individual track may turn out to be a combination of several retrackers and may depend on the sea state. The coastal high-frequency radar (HFR) ocean surface currents, hourly interpolated with a resolution up to $2 \mathrm{~km}$ and an offshore range up to $150 \mathrm{~km}$, are evaluated to validate the altimeter sea surface height (SSH) measurements. A method to derive HFR SSH mapped, with a varying spatial-scale optimal interpolation, from the HFR velocities has been implemented. Evaluated mainly in the regions farther than $25 \mathrm{~km}$ off the U.S. West Coast, the HFR SSH shows good agreement with Jason-1-2 altimetry products over the years 2008 and 2009. Three Jason-2 PISTACH retrackers and one generic open ocean retracker have been analyzed using the traditional 1 $\mathrm{Hz}$ sampling rate. Nearshore, an experimental reprocessing of the $20 \mathrm{~Hz}$ range measurements is also tested to check for a gain in along-track spatial resolution. Referencing to the HFR SSH indicate the need to have several retrackers available, even over the continental shelf, with Ice3 fitting better during Bloom events and MLE-4 (or Red3) for high sea states. These studies demonstrate the value of HFR as a potential tool to correct coastal altimeter SSH, refine their spatial resolution and provide some insight into the altimeter behavior as a function of ocean conditions.

Citation: Roesler, C. J., W. J. Emery, and S. Y. Kim (2013), Evaluating the use of high-frequency radar coastal currents to correct satellite altimetry, J. Geophys. Res. Oceans, 118, 3240-3259, doi:10.1002/jgrc.20220.

\section{Introduction}

[2] The ocean plays an important role in shaping global climate on a rapidly changing planet. There is a need to observe, understand and model its diverse mechanisms. With more than 20 years of experience, satellite altimetry is a mature technology over the open ocean. With the adequate constellation of satellites, multimission altimetry provides globally homogeneous, high resolution, and regular mapping of mesoscale sea level and ocean circulation variations [Morrow and Le Traon, 2006]. Yet, altimetry and its application still face many challenges in coastal regions. These shelf regions, with intense human interactions, have a special role from an economical and environmental as well as recreational and safety perspectives. With the increase of anthropogenic global climate change, this

${ }^{1}$ Colorado Center for Astrodynamics Research, Aerospace Engineering Sciences Department, University of Colorado, Boulder, Colorado, USA.

${ }^{2}$ Division of Ocean Systems Engineering, School of Mechanical, Aerospace and Systems Engineering, Korea Advanced Institute of Science and Technology, Yuseong-gu Daejeon, Republic of Korea.

Corresponding author: C. J. Roesler, Colorado Center for Astrodynamics Research, Aerospace Engineering Sciences Department, University of Colorado, Boulder, CO 80309, USA (carolyn.roesler@colorado.edu)

(C2013. American Geophysical Union. All Rights Reserved. 2169-9275/13/10.1002/jgrc. 20220 zone is susceptible to greater environmental stresses and natural hazards.

[3] The accuracy of the nadir-looking, pulse-limited satellite radar altimeter sea surface height (SSH) measurement degrades in coastal region. The geophysical (tides, dynamic atmospheric correction) and environmental (ionospheric, dry and wet tropospheric, sea state corrections) corrections, that need to be applied to the altimeter range, become less reliable and yet more variable [Andersen and Scharroo, 2011] Second, the altimeter waveform (return echo) becomes distorted. Coastal waters differ from the open ocean due to rapid changes in bathymetry on the continental slopes, shallow waters, and the presence of coastline boundaries. This induces greater variability resulting in shorter time- and space scales. Possible rapid changes in sea state and/or the presence of land within the altimeter footprint affect the shape of the waveform. Deng et al. [2002, 2003] observed that the waveforms from ERS-2 and TOPEX/Poseidon could be affected by land up to $20 \mathrm{~km}$ off the Australian coast. Furthermore, waveforms can be degraded by the presence of unrealistic high-radar return cross sections (Sig0) in the altimeter footprint, called "Sig0-bloom events" [Mitchum et al., 2004; Tournadre et al., 2006]. These Sig0-bloom events can occur from weak wind patches as well as surface slicks, which create a highly reflective specular surface. These "contaminated" waveforms will not conform to the shape of the standard 
ROESLER ET AL.: COASTAL RADARS TO CORRECT ALTIMETRY

open-ocean Brown model formulated by Brown [1977]. The ocean geophysical parameter ( $\mathrm{SSH}$, significant wave height (SWH) and Sig0 related to surface wind speed) retrievals from these waveforms (retracking), fitted to the Brown model, will be unreliable.

[4] Recovering these coastal altimetry data would be valuable for studies of the complex coastal circulation, sea level change, and the impact on this coastal circulation. Some of the reasons are that the long-term altimetric measurements are repeatable, stable, and are the only long-term coastal measurements available in some remote areas. At present, altimetry alone, even with corrected high-resolution along-track coastal data, will not resolve all the various time- and spatial scales of coastal dynamics. The revisit time (10 days for Jason-2; a cycle) and the distances between tracks $\left(\sim 100 \mathrm{~km}\right.$ at $40^{\circ}$ latitude for Jason-2) are too large, even with multiple altimeters. It must be considered as an important input to a coastal observing system. As such studies combining coastal altimetry and in situ data are adopted [Ruiz et al., 2009; Le Hénaff et al., 2010].

[5] Current altimetry products use generic open ocean processing that are retracked with the ocean Brown model and have been optimized for high-precision open-ocean variability along-track $1 \mathrm{~Hz}$ (or $\sim 7 \mathrm{~km}$ ground resolution) $\mathrm{SSH}$. There is a loss of data in coastal regions from stringent quality checks (distorted waveforms, nonavailable corrections, etc.) [Lee et al., 2009]. The use of altimeter data in the coastal zone requires the development of new retrackers, applying improved local corrections and reprocessing techniques to increase data coverage [Cipollini et al., 2008; Bouffard et al., 2008].

[6] These strategies have recently been implemented and demonstrated their values [Birol et al., 2010; Herbert et al., 2011]. The Coastal Altimetry (COASTALT) project provides experimental coastal data for several regions in the European seas (http://www.coastalt.eu). The Prototype Innovant de Système de Traitement pour l'Atimétrie Côtière et l'Hydrologie (PISTACH) coastal product [AVISO/Altimetry, 2010] is dedicated to the processing of Jason-2 altimeter data for the global coastal zone. But there are still many challenges to overcome for the full exploitation of coastal altimetry and their validation. Developing tools for the generation and quality check of these coastal products is a pertinent area of research. About 20 years of archived altimeter data in the coastal zones are waiting to be reprocessed.

[7] Improving the quality of altimeter geophysical retrievals is an important issue before using them for oceanographic applications. In this study, we will focus on the effect of retracking. Retracking is a key element to extend the use of coastal altimetry (whether pulse-limited or the new Cryosat-2 SAR/InSAR technology) and produces improved results in shallow-water tide modeling and sea surface topography determination [Hwang and Chen, 2000; Deng and Featherstone, 2006].

[8] Over the last few years, we have seen the development of new retrackers specific to coastal problems. Each has its own advantages and drawbacks. A review of waveform retracking methods can be found in Gommenginger et al. [2011]. To optimize the choice of the retracking method waveform, classifications are done [Deng and Featherstone, 2006] and are even included as a data ele- ment (waveform class) in the PISTASCH coastal product. However, there is a lack of a clear recommendation on which retracker to use depending on the situation. There is also a need to minimize the discontinuity of geophysical parameters from the open ocean to the coast. Changing retrackers from point to point along the track, depending only on the classification of the waveform, will create discontinuities from the relative biases between the various techniques. Consistency between the retrackers has to be investigated [Deng, 2004].

[9] The performance of retrackers can be estimated in a variety of ways. One possibility computes the statistics of residuals, using the geoid heights as a quasi-independent reference [Deng and Featherstone, 2006; Hwang et al., 2006]. But the geoid may not be well resolved in the coastal regions. The validation of coastal altimetry data has been performed using tide gauges [Lebedev et al., 2011] for the SSH, moored acoustic Doppler current profilers for near surface geostrophic velocity, and wave-rider buoys [Gómez-Enri et al., 2011] for the SWH measurements. The problem is that in these validations one compares sparse point measurements that may not lie exactly over the satellite track in a highly dynamic region. High-frequency radar (HFR) radial velocities perpendicular to the satellite track were used to estimate the quality of altimeter-derived velocity. The geometry of the HFR configuration, however, limits the number of collocations [Liu et al., 2012].

[10] This paper presents a novel approach to independently validate the coastal retrackers, using the HFR sea surface information that extends up to $150 \mathrm{~km}$ offshore along a continuous altimeter track and at the time of passage of the altimeter. The optimal method for altimetry retracking may turn out to be a combination of different retrackers for different parts of an altimeter track [Deng and Featherstone, 2006] or it may change for different cycles on the same track. The HFR data will ensure a continuity of the corrected altimetric SSH from the open ocean to the coast. These relationships will be explored in the west coast of the United States over the years 2008 and 2009, where the coverage of HFR surface current is excellent, with resolutions of 2 and $6 \mathrm{~km}$ depending on HFR-operating frequency. We will concentrate on Jason-1 and 2 data in this region (Figure 1) and on four retrackers, one conventional open-ocean retracker as well as three specific PISTACH retrackers. These four retrackers are available in the PISTACH data product and will hereafter be referred to as PISTACH retrackers for simplicity.

[11] Previous studies have compared altimetry and HFR surface current maps that support the potential of our methodology. Saraceno et al. [2008] show good correlations between a yearly time series of HFR velocities and an improved coastal SSH product at three locations along the Oregon coast. Two studies confirm that HFR contain more submesocale information [Chavanne and Klein, 2010; Kim et al., 2011] than present-day satellite altimetry. Consequently, the $2 \mathrm{~km}$ HFR data can help assess the feasibility of creating a higher-resolution coastal product by exploiting the higher frequency $20 \mathrm{~Hz}$ (or $\sim 330 \mathrm{~m}$ ground resolution) altimetric range rate measurements and implementing new editing and filtering techniques. This enhanced resolution coastal data set will better resolve the smaller scale of oceanographic processes in coastal zones. 


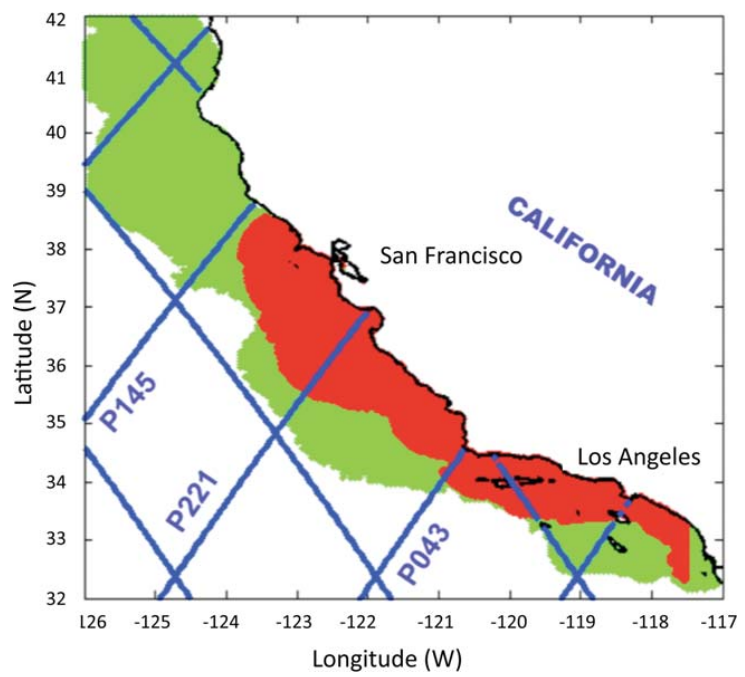

Figure 1. The U.S. West Coast HFR and altimeter data set coverage. Green corresponds to the HFR $6 \mathrm{~km}$ spatial resolution while the red is the $2 \mathrm{~km}$ resolution coverage. The blue lines are the ground tracks of the Jason-2 altimeter satellite.

[12] As part of the growing ocean observing infrastructure, HFR and altimeter data are complementary; through their respective instrument design, they observe different aspects of the coastal ocean. It is our goal, here, to fit HFR coastal currents to altimeter sea levels. The experience gained from a systematic comparison of both data sets, can provide hints on how to correct conventional coastal altimetry in regions where no HFR arrays are deployed and how to test the data quality of future altimetry missions, better suited for coastal observations.

[13] In this first study, which provides the basis for further in-depth investigations, the region of interest will be mainly $25 \mathrm{~km}$ till about $150 \mathrm{~km}$ (which represents the furthest extent of the HFR data) offshore. Our domain does not include the nearshore area, except briefly in section 4.2.2. This was chosen to reduce the errors in range correction that accumulate in the SSH altimetric estimates. Probably, a more specific HFR processing would be required in the nearshore region that includes costal boundaries. The potential of HFR to correct coastal altimetric heights can still be explored, because there are unresolved issues in retrieving altimetry range in this domain as aforementioned. For example, Lee et al. [2010] find that, on average for Jason-2 from July 2008 to July 2009, a retracker developed for nonocean surfaces improves the Brown-retracked SSH over the Californian continental shelf.

[14] The following questions will be addressed. How should we process the HFR surface current data to make them comparable with altimeter sea level measurements? How well are they related and what are the limitations of this comparison? Is there any information gained by validating the $2 \mathrm{~km}$ HFR data and high-frequency altimeter data? Can we use HFR to detect invalid segments of the traditional open-ocean retracked altimeter measurements and, if so, to decide which PISTACH retracker better fits the segment as well as evaluate the performance of the retrackers under various sea-state conditions?

[15] This paper will be organized as follows; section 2 presents the satellite altimetry and HFR data. In section 3, the methodology used to derive sea level measurements from HFR is explained. In section 4, the HFR SSHs are compared with altimetric SSH for three differently processed altimeter data sets as well as for several PISTACH retrackers. Examples of issues arising from several sea states are also examined. A discussion of the results, their limitations, and possible future extensions conclude the article in section 5 .

\section{Basic Principles and Data}

\subsection{Altimetry}

\subsubsection{Altimeter Data Sets}

[16] We use three different altimeter data sets all distributed by AVISO. The first one is the weekly multimission altimetry merged sea level anomaly (MSLA) product [AVISO/Altimetry, 2013], gridded SSHs computed with respect to a 7 year mean, on a $1 / 3^{\circ} \times 1 / 3^{\circ}$ Mercator grid. This product combines data from different missions. More specifically, we use the delayed-time, updated series. This data set usually has no values for offshore distances closer than about $20 \mathrm{~km}$, where the data has been flagged "bad" due to its proximity to land. We picked the weekly 2008 time series for the Californian coast in the region where we have coincident HFR currents.

[17] The second set is the global delayed-time alongtrack sea level anomalies (SLA) product [AVISO/Altimetry, 2012], which provides standard open-ocean $1 \mathrm{~Hz}$ (ground track spacing of $6 \mathrm{~km}$ ) along-track sea level anomalies computed with respect to a 7 year mean, with all standard corrections already applied. The time series is for the year 2008 for Jason-1, corresponding to cycles C220-C256 for the satellite-track P221, which terminates in Monterey Bay, California (Figure 1). Note that the number of this pass was for Jason-1 prior to its shift to the interleaved ground track in February 2009 and now corresponds to the Jason-2 pass denomination. Jason-type satellites have a 10 day repeat cycle.

[18] For the previous two sets, the corrections applied to the SSHs are from the regular open ocean processing; no specific coastal corrections have been applied.

[19] The third set is the Jason-2 PISTACH coastal product [AVISO/Altimetry, 2010]. For each correction affected by the proximity of land (such as the wet tropospheric correction), it offers a varied choice of correction scenarios. PISTACH also gives output for three new retracking schemes at the $20 \mathrm{~Hz}$ rate: Oce 3, Red3, and Ice 3. These three specific PISTACH retrackers will be analyzed together with one of the conventional Jason-2 deep-ocean retracker MLE-4. The latter is available in the PISTACH database and corresponds to the MLE-4 provided in the Sensor Geophysical Data Record (SGDR version "T" and the new SGDR version "D" (as of August 2012) [OSTM, 2011]).

[20] The Oce 3 retracker represents the output of MLE-3 performed on a denoised waveform, filtered after a singular value decomposition (SVD) [Severini, 2010]. The Red3 retracker also uses MLE-3 but is done on a restricted 
analysis window around the leading edge, to remove the eventual gates corrupted by the effects of land. The Ice3 retracker is a $30 \%$ threshold method [Davis, 1997], also implemented on a restricted analysis window.

[21] The PISTACH sea-level anomalies will be computed from the $20 \mathrm{~Hz}$ data stream for the various retrackers. For the specific coastal corrections we chose: the global ionospheric map (GIM) correction, the decontaminated water vapor correction where the microwave radiometer (MR) brightness temperatures are decontaminated from land before applying the water vapor retrieval algorithm [Desporte et al., 2007] and the tides from the FES2004 solution [Lyard et al., 2006]. The MLE-4 derived sea state bias (SSB) is applied to each retracker's range, because it is the only SSB field given in the PISATCH product. This assumption may not hold as each retracker behaves differently as a function the sea state. The $20 \mathrm{~Hz}$ SSB is linearly interpolated from $1 \mathrm{~Hz}$ measurements. The mean sea surface (MSS) from the Danish Space Center MSS DNSC08 is used, but this is not so critical, because we remove a mean of the time series for our final sea-level heights product. All other corrections are the standard ones. The waveforms are extracted from the corresponding SGDR product.

[22] For the PISTACH sets, the time series along P221 is analyzed from August 2008 until the end of December 2009, corresponding to cycles C004-C054 (two cycles $\mathrm{C} 005$ and $\mathrm{C} 018$ are not included). The PISTACH data will be processed in two different manners as described in section 4.2 .

\subsubsection{Altimeter Waveforms}

[23] Conventional satellite altimeters are nadir-pointing instruments that emit short pulses reflected by the sea surface. The geometry of the footprint is pulse limited, and pulse compression is used to achieve high-accuracy ranging. The time evolution of the echo, the waveform, represents the mean return backscattered power as a function of time. To reduce speckle, the individual return echoes are averaged onboard, typically over 100 successive echoes (at $\mathrm{Ku}$-band) over a period of $50 \mathrm{~ms}$. These $20 \mathrm{~Hz}$ waveforms are transmitted to the ground, where retracking (ground retracking) is applied to refine the extraction of the oceanic parameters. For open-ocean generic products, the data are averaged to $1 \mathrm{~Hz}$ [Chelton et al., 2001].

[24] Over water, after the pulse hits the surface, the illuminated surface area grows from a point to a disk and then spreads as an annulus increasing in diameter but with a constant surface area. The corresponding waveform shows a characteristic shape with a sharp rise to a maximum level (leading edge) followed by a gradually sloping trailing edge, as the off-nadir signal slowly reaches the edge of the radar beam. The waveform provides the range between the satellite and the surface at nadir via the two-way travel time of the transmitted pulse, the SWH via the slope of the leading edge and the backscattering coefficient Sig0, which represents the surface roughness via the returned power (Figure 2). This ocean shape can be represented by an analytical Brown [1977] model. For the Jason-1 and Jason-2 altimeters, considered in this study, the waveforms of 104 samples (or range gates) are retracked using a maximum likelihood estimator (MLE) fit to the Brown model. The MLE-3 retrieves three geophysical parameters (range,

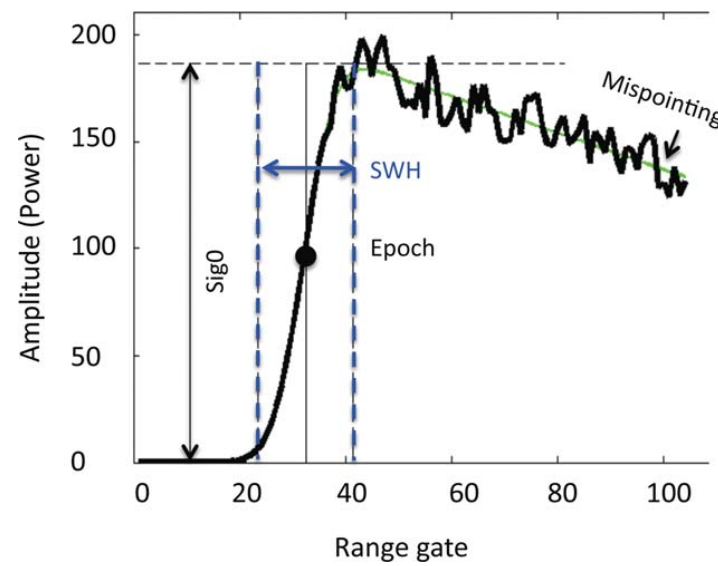

Figure 2. Jason-2 $\mathrm{Ku}$ Band Echo (in black). Brown waveform (in green) and parameters retrieved by MLE-4.

SWH, and Sig0); the MLE-4 also estimates the antennamispointing angle (slope of the trailing edge).

[25] The Brown model has been derived from the physical properties of a rough and homogeneous scattering surface for near-normal incidence. Although, different refined analytical forms exist, one of the main assumptions related to the surface properties (not the instrument, or pulse shape) are that the sea surface is homogeneous over the footprint and that the probability distribution function of the surface slope and elevation has a predefined shape, essentially Gaussian. When this is not the case, the waveform will not conform to the Brown model, and new retracking strategies will need to be implemented.

[26] The altimeter waveforms may be corrupted by nonuniform radar return Sig0 in the altimeter footprint. In the case of Sig0-blooms, there are occurrences of unusually high Sig0 due to highly reflecting ocean patches. The presence of these higher Sig0 values may signal a breakdown in the typical Brown open ocean waveform model (Figure $3)$. First of all, the onboard tracker normally centers the waveform leading edge at a predefined gate range ( 32.5 for Jason-2) to keep the waveform well centered in the analysis window. But with distorted waveforms the leading edge can shift. This can be observed in the consecutive $20 \mathrm{~Hz}$ waveform series of Figure $3 \mathrm{a}$, in the presence of a Sig0bloom around $30 \mathrm{~km}$ off the coast, for cycle 26 on P221 (as well as nearshore, for waveforms contaminated by land). Second, Sig0-blooms can create various waveform shapes. The distortion is not predetermined (Figures $3 c$ and $3 d$ ): the trailing edge slope could be increasing or decreasing; the peakiness increased; there could be the presence of a V-shape or round pattern similar to the ones observed during rain events [Quartly et al., 1998].

[27] The waveforms affected by land will not be included in this study. The interested reader can refer to Gommenginger et al. [2011]. For completion, we will mention that for an ocean to land transition, the altimeter footprint will gradually contain more and more land returns. More waveform samples will progressively be perturbed starting from the trailing edge and moving toward the leading edge. The shape of the coastline, the relief, and the 
a)

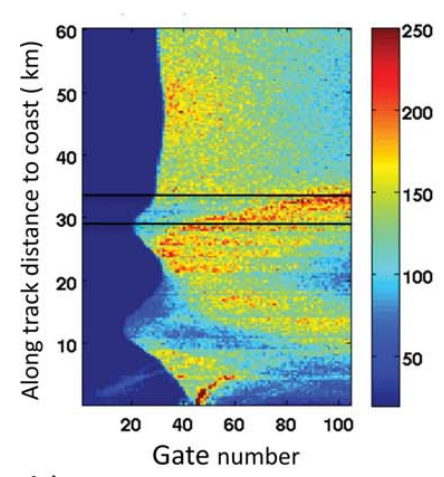

b)

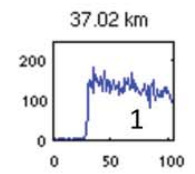

c)
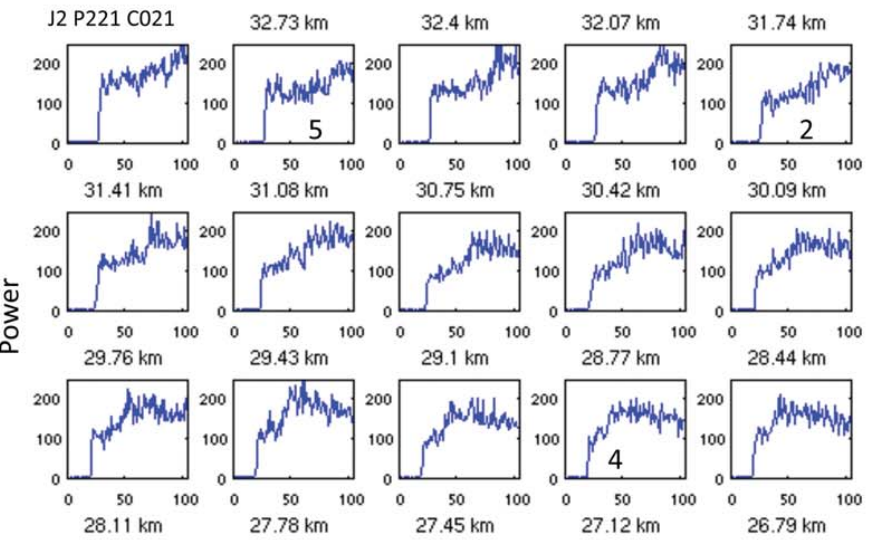

d)
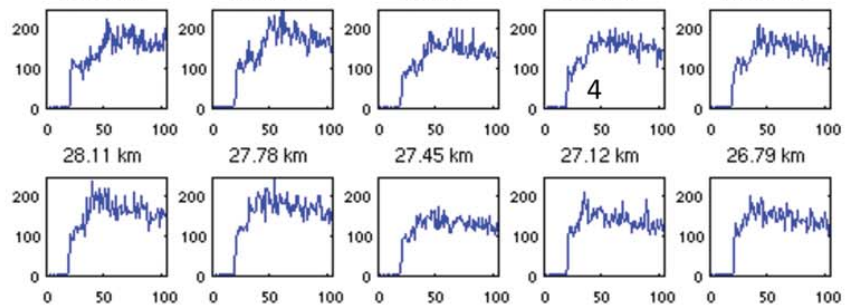

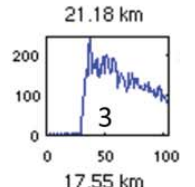

Gate number

Figure 3. (a) The $20 \mathrm{~Hz}$ waveforms from Jason-2 Cycle 26 pass 221, 3 February 2008; (b) typical Brown waveform; (c) consecutive $20 \mathrm{~Hz}$ waveforms, $30 \mathrm{~km}$ off shore in the presence of a Sig0-bloom event, over the region indicated by a black box in Figure 3a. (d) Other "bloom" waveform shapes at 21.18 and $17.55 \mathrm{~km}$. (1) Brown waveform, (2) increasing trailing edge, (3) peakiness increased, (4) round pattern, (5) V-shape.

backscattering properties of the terrain will produce a variety of coastal waveform shapes.

[28] Furthermore, over the shelf regions, the ocean characteristics are expected to change and have smaller spatial scales. There could be a variety of waveforms affected in a yet not well-defined way, because the dynamics of the system are still not well understood.

\subsubsection{Altimetric Sea-Level Height Corrections}

[29] Retracking improves the estimate of the range, but it still needs to be adjusted for atmospheric path delays (dry/ wet and ionospheric) as well as for an electromagnetic bias $\mathrm{SSB}$. These can be problematic in the littoral regions. The Jason altimeters carry onboard MRs to correct for the water vapor but close to shore the MR footprint will be contaminated by land. Due to technical improvements the advance microwave radiometer (AMR), onboard Jason-2, water vapor estimates are probably not corrupted by land until $\sim 25 \mathrm{~km}$ offshore relative to $50 \mathrm{~km}$ for the Jason-1 MR. For the highly variable in time and space water vapor corrections, different strategies exist, such as correcting the altimeter radiometer due to land contamination [Desporte et al., 2007; Brown, 2010]. The frequency-dependent ionospheric path delay is calculated from the dual-frequency altimeter (C-band and $\mathrm{Ku}$-band); but land also contaminates their footprints (C-band has a larger beam width). The ionospheric correction GIM derived from the global positioning system (GPS) network is recommended in coastal areas.

[30] The SSB correction compensates for the bias of the altimeter range measurement toward the troughs of the ocean waves, as well as for an instrumental bias. It depends on the sea state (wave types and wind field). The open ocean SSB is empirically determined from the SWH and the wind speed [Tran et al., 2006]. In the coastal zone, with complex wind and wave dynamics, this empirical relationship may not be valid. However, we have opted to include it.

[31] Finally, once the range has been corrected, the SLA is computed relative to a MSS level:

$$
\begin{aligned}
\text { SLA }= & \text { Satellite height }- \text { Corrected range }- \text { MSS } \\
& - \text { Tides height }- \text { Atmospheric pressure loading, }
\end{aligned}
$$

where Corrected range $=$ Altimeter Range

$$
+ \text { Atmospheric corrections }+ \text { SSB. }
$$

[32] Other important considerations in the coastal regions are the tidal and high-frequency atmospheric pressure loading corrections that are less accurate. All of the environmental aforementioned corrections are subjects of ongoing research.

[33] Depending on the altimeter data set used and the focus of the analysis (more on the open ocean or on the littoral regions), some improved corrections will, or will not, be implemented. It is important to acknowledge these problems so that we understand that the altimeter segments could be affected by these corrections and, each one of them, in variable amounts and at variable distances from the shoreline.

\subsection{High-Frequency Radar}

[34] Operational shore-based HFR systems provide hourly surface current maps averaged within the upper meter depth, with an offshore range of $50-150 \mathrm{~km}$ and a spatial resolution of $0.5-6 \mathrm{~km}$, depending on the radar operating frequency [Barrick et al., 1977; Lipa and 


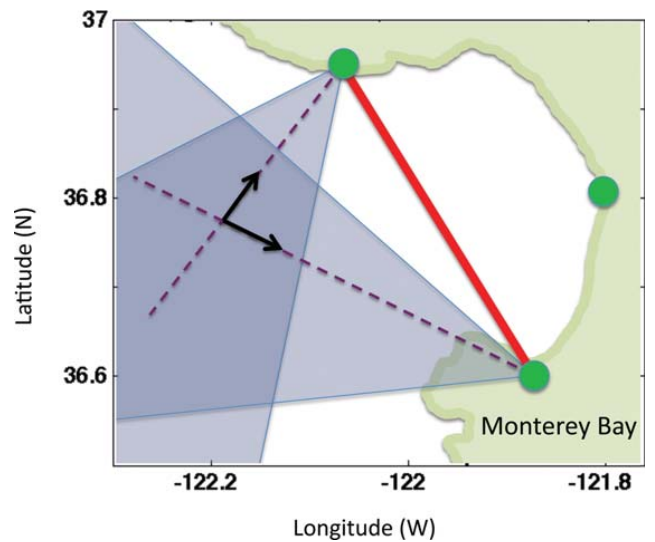

Figure 4. HFR geometry in Monterey Bay. Green dot: HFR radar sites. Red line: Baseline between two stations. Blue zone: part of HFR coverage area. Dotted line: radial line. Vector: radial surface current.

Barrick, 1986; Ohlmann et al., 2007]. The HFR emits a high-frequency radio signal in the range of 5-25 $\mathrm{MHz}$, which are backscattered from the ocean surface. The ocean gravity waves with a wavelength of half the transmitted wavelength (Bragg scatter) will reflect back coherently and result in a strong peak in the retuned energy spectrum. The Doppler shift of the peak indicates movement of these gravity waves in a direction either toward or away from the HFR site (radial). The ocean gravity waves have a known phase velocity and ride on the surface current. Subtraction of the theoretical phase velocity gives the radial ocean surface current velocity [Paduan et al., 1997].

[35] Individual HFR reports the surface radial velocity map, which is a set of projected velocity components of the true current field with respect to the radar-bearing angles. Thus, in order to extract a vector current map, multiple radial velocity maps are required (Figure 4). The geometry of the HFR sites defines the coverage where the current estimates are reliable. For instance, the baseline is a straight line between two radars [Paduan et al., 1997]. Along the baseline, the current estimate can be limited as the radial velocities from the two sites are nearly parallel. However, the postprocessing of the HFR radials can eliminate most of the artifacts along the baseline [Kim et al., 2008, 2011].

[36] The uncertainty in the HFR-derived surface current measurements can be influenced by several factors such as antenna beam pattern, signal-to-noise ratio (SNR), sea state related to wind speed and direction, radar geometry, and interference of radar frequency. For example, the locally calibrated radar beam pattern can improve the quality of radial maps and help to produce the realistic current field [Paduan et al., 2006]. Moreover, the low SNR of Bragg scatter echo due to weak wind condition can hinder to estimate radial solutions accurately. The baseline due to radar geometry can be placed on land or in the sea, which may generate spurious vector solutions in the near-coast regions. The uncertainty estimated from both independent observations and HFR radial velocity measurement itself ranges from 3 to $12 \mathrm{~cm} / \mathrm{s}$ (see Laws and Paduan [2011] and Kim et al. [2011] for more details).
[37] In this paper, we analyze the optimally interpolated HFR-derived surface currents off California coast for 2 years (2008-2009) with the resolution of 2 and $6 \mathrm{~km}$ in space and hourly in time [Kim et al., 2008; Kim, 2010]. The $2 \mathrm{~km}(6 \mathrm{~km})$ resolution HFR data have a minimum nearshore range of $3 \mathrm{~km}(9 \mathrm{~km})$ and maximum offshore range of $50 \mathrm{~km}(150 \mathrm{~km})$ (Figure 1).

\section{Analysis Method: Retrieval of Synthetic SSH From HFR Currents}

[38] Altimetry maps the vertically integrated $\mathrm{SSH}$ that can be related to the geostrophic flow, whereas HFR data gives us a surface total velocity and includes ageostrophic processes that need to be removed to compare the two data sets. In this study, an optimal interpolation (OI) was chosen to retrieve the geostrophic currents from the total velocity surface measured by the HFRs. More precisely, the OI estimates the nondivergent stream function and, assuming near-geostrophy for the associated nondivergent current field, the HFR two-dimensional SSH field. The OI requires an analysis of the time- and spatial scales of the coastal oceanic features. These features are expected to vary with the distance to the coastline and/or with the bathymetry and thus may vary regionally.

\subsection{Optimal Interpolation}

[39] The variable to be estimated is the stream function $\psi$, because we are interested in capturing the geostrophic (nondivergent) part of the HFR-observed flow. If the flow is assumed to be nearly geostrophic, there is a linear relationship between the stream function and the observed velocities: $(u=-\mathrm{d} \psi / \mathrm{d} y$ and $v=\mathrm{d} \psi / \mathrm{d} x)$. The stream function $\psi$ can, then, be calculated directly from the HFRderived currents using an OI [Bretherton et al., 1976; Wilkin et al., 2002].

[40] The velocity observations are concatenated in the data vector $\phi^{\text {obs }}=\left[\begin{array}{ll}\mathbf{u} & \mathbf{v}\end{array}\right]^{\mathrm{T}}\left({ }^{\mathrm{T}}\right.$ denotes the vector transpose), where $\mathbf{u}$ and $\mathbf{v}$ refer to the suite of measurements $\left(u_{i}\right)$ and $\left(v_{i}\right)$ done at distinct locations with velocity components $\left[u_{i}\right.$ $\left.v_{i}\right]^{\mathrm{T}}$. The observations are inexact, $\phi_{i}{ }^{\mathrm{obs}}=\phi_{i}+e_{i}$, where $\phi_{i}$ is the true value and $e_{i}$ is the measurement error, assumed to be uncorrelated with each other. The vector stream function estimate at the OI grid point locations is given by

$$
\psi^{\text {est }}=C_{\mathrm{md}}\left(C_{\mathrm{dd}}\right)^{-1} \phi^{\text {obs }}
$$

where $C_{\mathrm{md}}$ is the covariance of the estimated model with the data:

$$
\left(C_{\mathrm{md}}\right)_{\mathrm{ki}}=\left\langle\psi_{k}^{\text {est }} \phi_{i}^{\text {obs }}\right\rangle=\left\langle y_{k}{ }^{\text {est }} \phi_{i}\right\rangle
$$

[41] ( \langle\rangle is the expected value) $C_{\mathrm{dd}}$ is the covariance of the data with each other:

$$
\left(C_{\mathrm{dd}}\right)_{i j}=\left\langle\phi_{i}{ }^{\mathrm{obs}} \phi_{j}^{\mathrm{obs}}\right\rangle=\left\langle\phi_{i} \phi_{j}\right\rangle+\left\langle e_{i} e_{j}\right\rangle=\left\langle\phi_{i} \phi_{j}\right\rangle+e^{2} \delta_{i j}
$$

where $e^{2}$ is the noise-error variance of the surface currents.

[42] The uncertainty covariance matrix of the estimate is defined as 


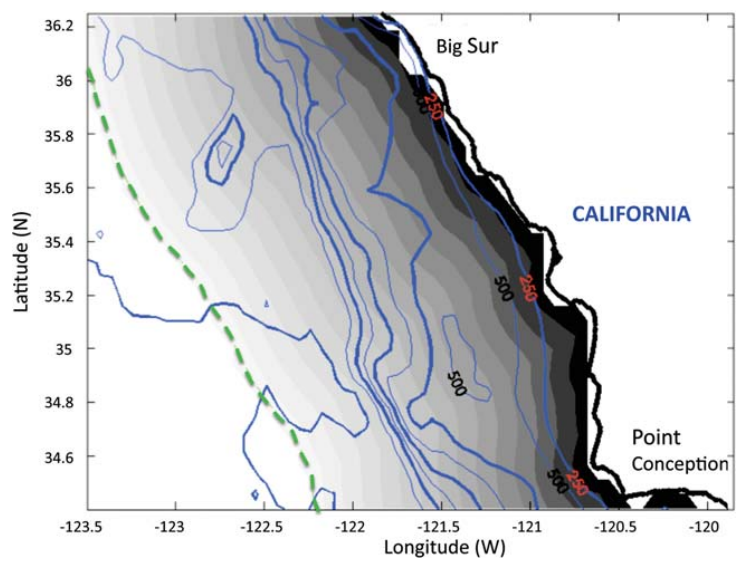

Figure 5. Data set geography between Big Sur and point conception. Gray scale: $10 \mathrm{~km}$ distance-band regions from $150 \mathrm{~km}$ to the coast. Blue contours: bathymetry with an interval of $500 \mathrm{~m}$ from 500 to $4000 \mathrm{~m}$; bold lines every $1000 \mathrm{~m}$ from 1000 to $4000 \mathrm{~m}$; light lines every $1000 \mathrm{~m}$ from 500 to $3500 \mathrm{~m}$. Dotted green line: boundary of HFR 6 $\mathrm{km}$ resolution.

$$
\xi=s_{\psi}^{2 \cdot} I-\left(C_{\mathrm{md}}\right)\left(C_{\mathrm{dd}}\right)^{-1}\left(C_{\mathrm{md}}\right)^{t}
$$

where $s_{\psi}{ }^{2}$ is the variance of $\psi$ and $I$ is an identity matrix.

[43] At this preliminary stage, we will assume that the low-frequency parts of the velocity components are nearly geostrophic [Tisch et al., 1989; Chereskin and Trunnell, 1996] The hourly HFR velocities contain HF components arising from tides and short-term wind events that need to be removed in order to better approximate the observed underlying geostrophic flow. This will de done through temporal averaging. The study of the velocity temporal scales over the coastal transition zone will provide the information about how much temporal averaging can be done, while still preserving the local structure that is required to compare with the instantaneous altimeter measurements. Finally, we need to find a functional form of the various spatial covariance functions derived from the observed HFR velocity spatial scales and structures.

\subsection{Data Covariance Scales}

[44] The first analysis was done along the southern California coast between Big Sur and Point Conception, where the coastline is relatively straight (Figure 5). For this analysis, we are using a 3 day running average of a 2008 time series sampled every 3 days, from hourly HFR measured ocean surface currents resampled and postprocessed to 6 $\mathrm{km}$ resolution. Three-day composites have been selected to remove the tidal and inertial current components in these currents. To depict how the time- and space scales vary with the distance to coast, the HFR velocity covariances have been analyzed for fifteen $10 \mathrm{~km}$ distance-band regions from $150 \mathrm{~km}$ offshore to the coast (Figure 5). The velocities have been projected on to across-shelf velocities, $u$; and alongshelf velocities, $v$; corresponding to the acrossshelf axis $X$, and alongshelf axis $Y$, respectively. A 2008 seasonal mean has been removed from the data, corre- sponding to the two characteristic current patterns of the California coastal current system.

[45] The HFR temporal covariances were analyzed over each $10 \mathrm{~km}$ wide region and averaged over the year 2008 . A decrease of the $e$-folding timescale from about 10 days in the open ocean to 3 days closer to shore can be detected (Figure 6). Over the complete domain, the data will de relatively highly correlated over the lowest timescale, which is 3 days. Hence, as a first approximation of the observed geostrophic flow we will use a 3 day averaging of the HFR velocities. This may be less representative of conditions in the near-coastal region due to smoothing; but for this first exploration, which compares altimetry and HFR, mainly, on coastal regions further than $25 \mathrm{~km}$ offshore, this level of smoothing should still be adequate. This 3 day temporal averaging was also chosen in the deep ocean by Bretherton et al. [1976] and Wilkin et al. [2002]. We acknowledge that averaging over 1 or 2 days should also be tested in the future. For example, using HFR velocities, several authors average over 2 days to retrieve the low-frequency subinertial currents [Chavanne and Klein, 2010; Saraceno et al., 2008].

[46] Also for submesoscale structures of the order of 10 $\mathrm{km}$, the Rossby radius of deformation may be approaching 1 and the advective terms cannot be neglected. Nevertheless, even if the observations are divergent, the divergence in the data will be removed by applying this specific OI gridding algorithm, which enforces nondivergence. The validity of the assumption can then be assessed by comparing the observations and the derived gridded geostrophic currents.

[47] Next, the HFR velocity spatial covariances are estimated by computing the data spatial covariance values at zero time lag. The spatial covariances are binned according to spatial lags across-shelf $X$ and alongshelf $Y$, normalized relative to the maximum, and averaged over the year of 2008 (Figure 7) for each $10 \mathrm{~km}$ wide band. The blank places in (Figure 7) represent areas of no data coverage; the coast is on the right, the open ocean is on the left. The features in Figure 7 make reasonable sense. The length scale of the across-shelf covariance $\left(C_{\mathrm{uu}}\right)$ grows in both directions $(X$ and $Y)$ with increasing distance offshore. This is consistent with the coastal boundary limiting eddy size and is the theoretical pattern for coastally trapped waves across-shelf velocity. We also observe, more or less, the same pattern in the alongshelf covariance $\left(C_{\mathrm{vV}}\right)$ especially in the central small spatial-lag area (central red area in Figure 7). Although, in contrast to $C_{\mathrm{uu}}$, there is a component that stays elongated in the $Y$, alongshelf direction, visible in the slightly larger spatial-lag area (yellow area in Figure 7). Again, this is consistent with coastally trapped wave theory. The distance from the coast does not appreciably affect the alongshelf scale for the alongshelf velocity.

[48] For the purpose of this study, we will fit a theoretical spatial covariance function to the central (red) section and assume that $C_{\mathrm{vv}}$ grows in both directions with increasing distance offshore. By making this approximation and from the structures of both observed spatial covariances, we can then assume the velocities to be consistent with the statistics of a locally homogeneous, isotropic turbulence. This implies that the stream function spatial covariance $C_{\psi \psi}$ is related to the velocity spatial covariances 
ROESLER ET AL.: COASTAL RADARS TO CORRECT ALTIMETRY
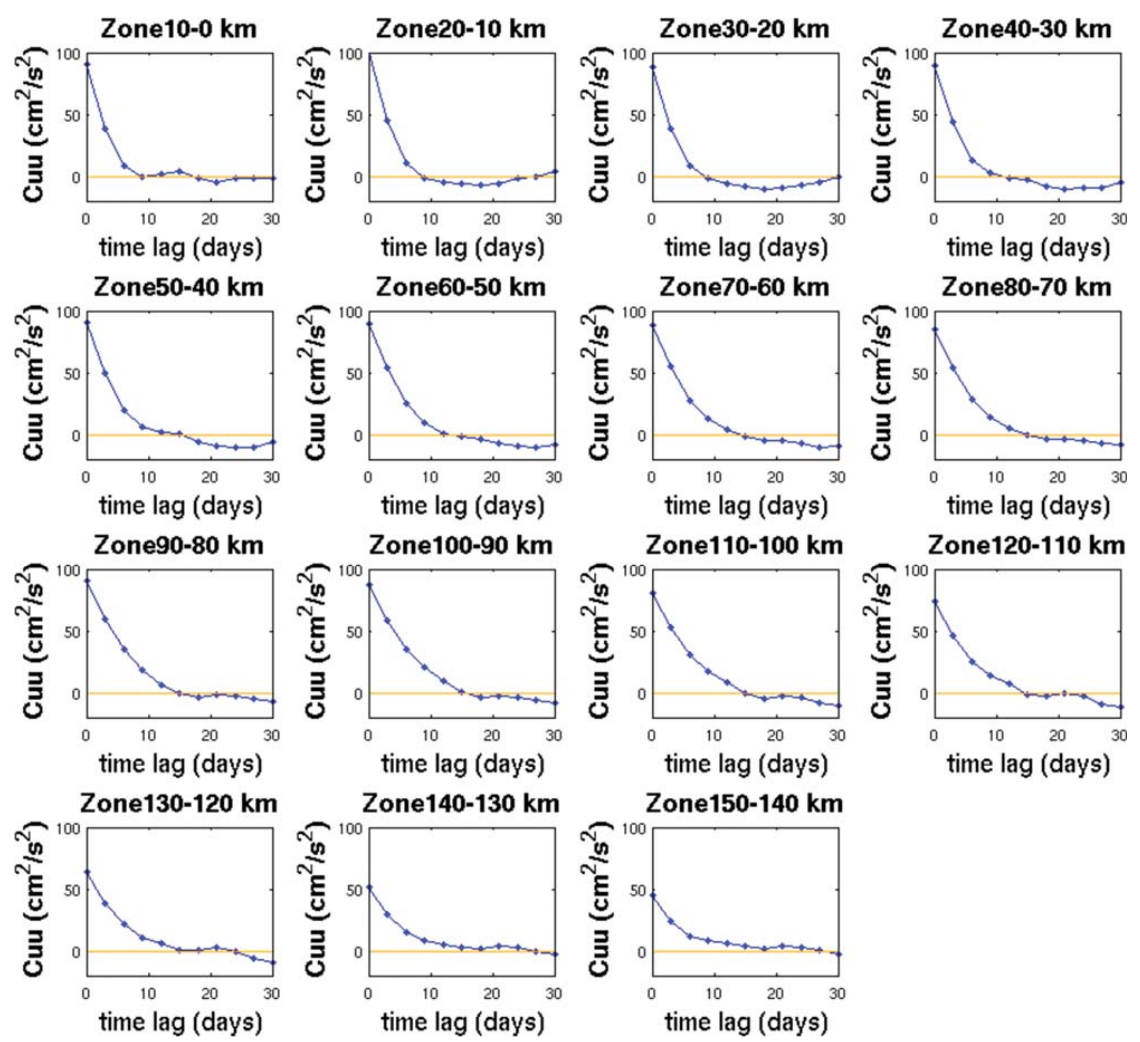

Figure 6. Temporal covariance of the across-shelf $(u)$ HFR velocity data at zero spatial lag, averaged over the year 2008 for each $10 \mathrm{~km}$ width region. The offshore region increases from top to bottom and left to right.

[Bretherton et al., 1976; Wilkin et al., 2002]. We use the Walstadt et al. 1991] stream function $\psi$ for the normalized spatial covariance defined as

$$
C_{\psi \psi}=\left(1-r^{2} / b^{2}\right) \exp \left(-r^{2} / a^{2}\right)
$$

from which the theoretical velocities covariances can be derived [Wilkin et al., 2002]

$$
\begin{array}{ll}
C_{\mathrm{uu}}=\left(X^{2} / r^{2}\right)(T-S)+S & \\
C_{\mathrm{vv}}=\left(Y^{2} / r^{2}\right)(T-S)+S \text { where } & T=-1 / r\left(\partial C_{\psi \psi} / \partial r\right) \\
C_{\mathrm{uv}}=\left(X Y / r^{2}\right)(T-S) & S=-1 / r\left(\partial^{2} C_{\psi \psi} / \partial^{2} r\right) \\
C_{\psi \mathrm{v}}=Y T & r=\left(X^{2}+Y^{2}\right)^{1 / 2} \\
C_{\psi \mathrm{v}}=-X T &
\end{array}
$$

[49] Figure 8 shows the good match between the observed and fitted covariances for the offshore region between 50 and $60 \mathrm{~km}$, normalized and averaged over the year 2008. We fit the parameters $a$ and $b$ in equation (1) for each zone. We find, for example, in the offshore zone between 50 and $60 \mathrm{~km}, a=50 \mathrm{~km}$ and $b=70 \mathrm{~km}$ and in the offshore zone between 20 and $30 \mathrm{~km}, a=35 \mathrm{~km}$ and $b=50 \mathrm{~km}$

[50] In conclusion, we can directly estimate the stream function, proportional to the SSHs (assuming geostrophy), from the observed HFR currents with an OI method that uses locally varying spatial scales depending on the distance of the OI grid point from the coast. The covariance matrices $C_{\mathrm{md}}$ and $C_{\mathrm{dd}}$ in equation 1 are:

$$
C_{\mathrm{dd}}=\left(\begin{array}{ll}
C_{\mathrm{uu}}+\mathrm{e}^{2} I & C_{\mathrm{uv}} \\
C_{\mathrm{uv}} & C_{\mathrm{vv}}+\mathrm{e}^{2} I
\end{array}\right) \quad \text { and } \quad C_{\mathrm{md}}=\left(C_{\psi \mathrm{u}} C_{\psi \mathrm{v}}\right)
$$

[51] In this OI implementation, we incorporated a linear change in spatial scale over the continental shelf. Thus, each point in the domain of interest is assigned a specific spatial scale depending on its distance from the coast. We selected observations in an area with a radius selected as the local spatial scale to reduce the amount of observations and the computational time.

[52] The velocity-noise error for the HFR is assumed to be constant $e=15 \mathrm{~cm} / \mathrm{s}$, although in reality, this error varies depending primarily on the radar and current geometry, less on weather conditions and in our case on how well the initial geostrophic assumption is satisfied. This value was chosen considering the typical errors found in the HFR velocities (section 2). From a total velocity error of $15 \mathrm{~cm} /$ $\mathrm{s}$, the individual error component could be lowered, and after a 3 day averaging, the errors could decrease even more, if the geostrophic component is properly captured. 

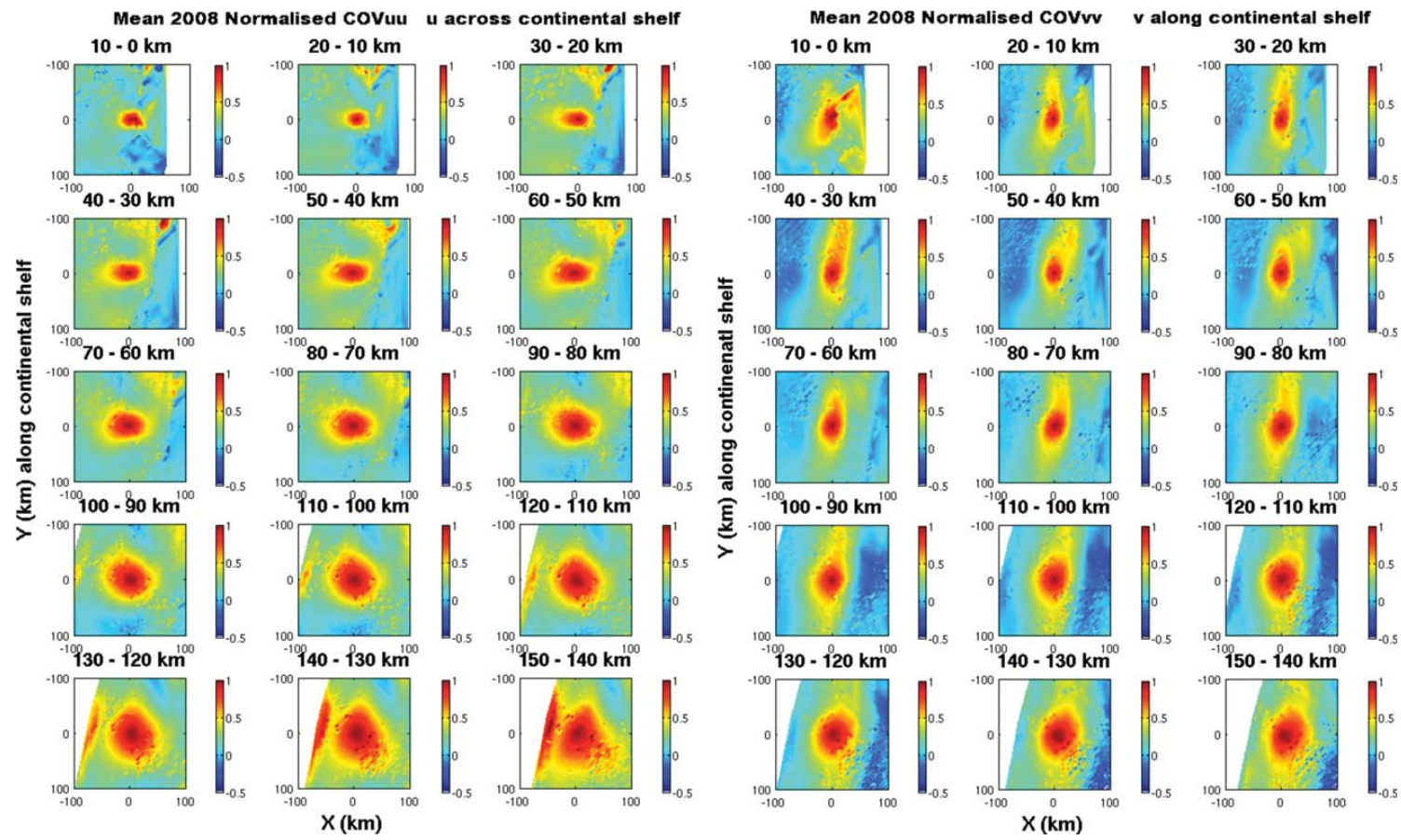

Figure 7. Covariances of HFR velocity (left) $C_{\mathrm{uu}}$ and (right) $C_{\mathrm{vv}}$ at zero time lag, for each $10 \mathrm{~km}$ wide region, binned according to spatial lag $X$ (cross-shelf) and $Y$ (alongshelf), normalized and averaged over 2008. The seasonal mean has been removed from the across-shelf velocity, $u$, and along-shelf velocity, $\mathrm{v}$. The offshore region increases from top to bottom and left to right.

Furthermore, the residuals of the optimally interpolated field should be consistent with the assumed error variance of the data. Over 2008, we find that the root-mean-square (RMS) difference of the HFR and the OI-mapped velocities in the $u$ and $v$ components are 3.9 and $4.4 \mathrm{~cm} / \mathrm{s}$ less than the estimated error variance of $15 \mathrm{~cm} / \mathrm{s}$. Also an RMS error of $10 \%$ in the HFR velocity observations results in an RMS error of about $4 \mathrm{~cm} / \mathrm{s}$ in the OI-derived geostrophic velocities, which is, again, smaller than $15 \mathrm{~cm} / \mathrm{s}$ (even if we take into account the factor of 2 (section 4 )).

[53] We implemented this OI mapping method for the region of the Jason-1 track P221 that terminates in Monterey bay (Figure 9). The bathymetry found along this area of the California coast causes the formation of eddies [Ikeda et al., 1984; Hickey, 1998; Strub et al., 1991]. This is a region prone to having large sea level anomaly (SLA) variations and a good one to test our methodology. In Figure 10 , we present the results of the OI from the $2 \mathrm{~km}$ resolution HFR velocities, for the along-track pass P221 on 6 September 2008. The mapped SSH has been computed using a varying spatial scale (Figure 10, right) versus using a single spatial scale, chosen to be the one for the zone between 50 and $60 \mathrm{~km}$ over the continental shelf (Figure 10 , left). The varying spatial-scale method clearly shows more details in the dynamic height structure.

[54] The OI methodology was explained for the more complex case of a direct comparison of HFR observations with the instantaneous altimeter along track on an OI grid of 2 or $6 \mathrm{~km}$ resolution. But we will also adapt the method- ology to compare HFR data with the weekly MSLA product (section 2.1.3) on a $1 / 3^{\circ} \times 1 / 3^{\circ}$ grid. This is simpler. First, we can directly average the HRF currents over a week, the same time sampling. Second, the OI grid is chosen to be the same as the MSLA grid, thus about $30 \mathrm{~km}$ resolution. This resolution imposes a limit on the spatialscales features that can be detected to more than $60 \mathrm{~km}$. So, in this case, we will only use one spatial scale chosen to be the one for the zone $50-60 \mathrm{~km}$.

[55] Note that the HFR synthetic SSHs contain an unknown bias due to the geostrophic relationship $(u=-g / f$ $\partial \mathrm{SSH} / \partial y$ and $v=g / f \partial \mathrm{SSH} / \partial x$ ), where $f$ is the Coriolis parameter and $g$ is the gravitational acceleration.

\section{Results}

[56] The HFR synthetic SSHs are compared with altimetric SSH for three differently processed altimeter data sets as well as for several PISTACH retrackers. Examples of issues arising from various sea states are also examined.

[57] In order to obtain consistent time series of HFR and altimeter SLA, i.e., relative to the same reference level, the mean of the sea level time series is removed from both SSH data sets. This mean is adjusted for each specific data set used. Also we notice that, in general, to match the variations of the altimeter SLA, the HFR sea levels need to be amplified by a factor of 2 . We think this is due to the smoothing inherent in the OI methodology, but the exact reason for this discrepancy requires further investigation. 


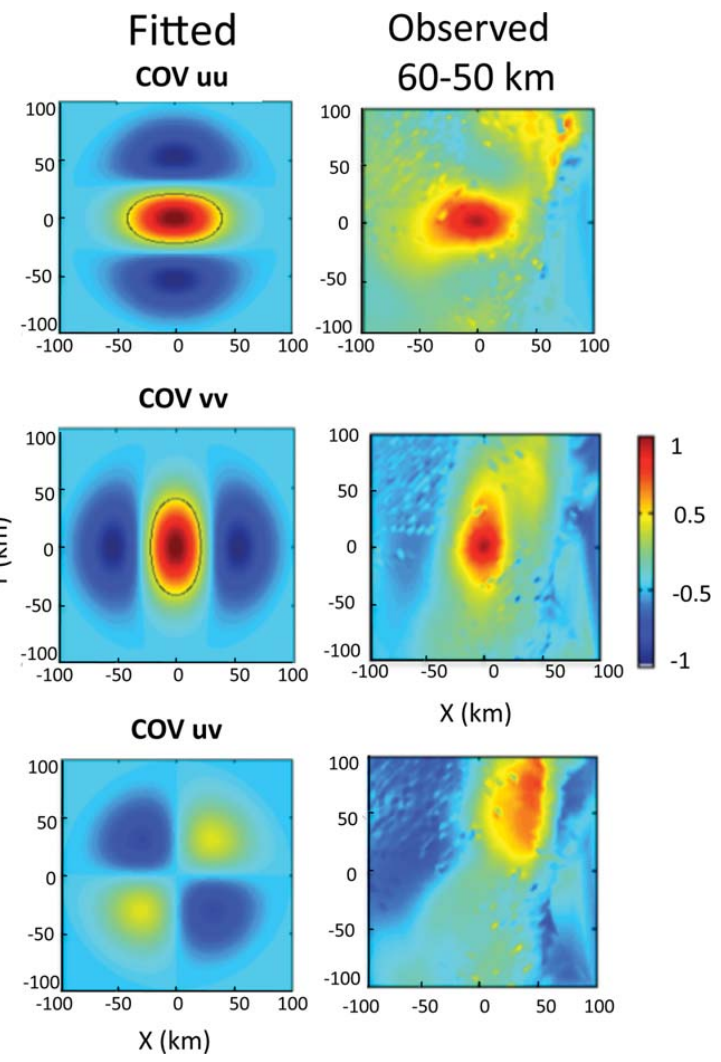

Figure 8. (right) Observed and (left) fitted covariance functions for the across-shelf $(u)$ and alongshelf $(v)$ HFR velocities in the $60-50 \mathrm{~km}$ offshore zone.

This estimated factor of 2 has been derived from the least squares method (refer to section 4.1.2 for details) and is applied to each HFR-inferred sea level set.

\subsection{Comparison of HFR Over the Open Ocean With Standard Altimetry Product}

[58] The comparison of HFR and altimeter SLA over the wide continental shelf, which we refer to as the open ocean

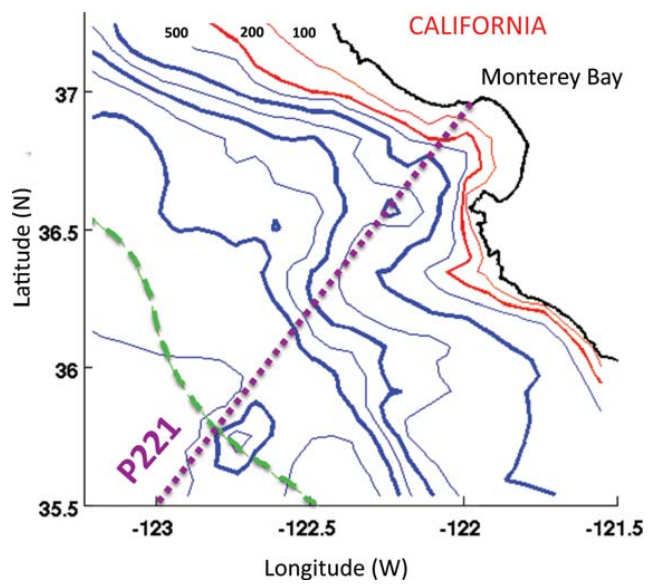

Figure 9. Monterey Bay bathymetry. Red lines: bathymetry, light $100 \mathrm{~m}$, bold $250 \mathrm{~m}$. Blue lines: bathymetry, bold every $1000 \mathrm{~m}$ from 1000 to $4000 \mathrm{~m}$, light every $1000 \mathrm{~m}$ from 500 to $3500 \mathrm{~m}$. HFR limit: long dotted line. Jason-2 P221: small dotted line.

(25-150 km offshore), will set the criteria for the feasibility of the method. This is an area where altimetry is assumed to be reliable.

\subsubsection{HFR and MSLA}

[59] First of all, we will analyze the weekly MSLA over the year 2008 with the corresponding weekly HFR synthetic SLAs, derived from the OI, on a $1 / 3^{\circ} \times 1 / 3^{\circ}$ grid for the Californian coastal region. The mean of the sea level time series is removed from both data sets. To adjust for the unknown bias for each weekly HFR sea levels (derived from the OI method), an estimate of the bias over the complete region is computed, and then subtracted. The bias for week $\mathrm{w}$ is computed by taking the mean of the difference between the HFR and MSLA sea levels, over the entire region.

[60] We created a movie for the weekly time series, of both sets presented as snap shots in Figure 11 with a sampling of every 6 weeks. After August 2008, the field

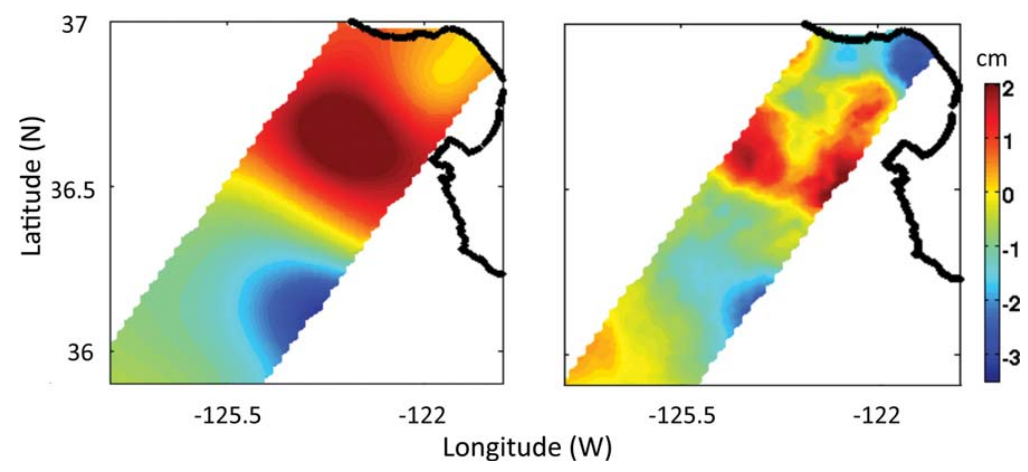

Figure 10. HFR synthetic SSH in centimeters, computed at the Jason-2 time on 6 September 2008 along-track P221. Effect of using a single spatial scale chosen at the (left) $50 \mathrm{~km}$ zone and the (right) varying spatial scale OI method, on a $2 \mathrm{~km}$ resolution grid. The computation time is faster for the single spatial-scale method, so the output area is slightly larger. 
ROESLER ET AL.: COASTAL RADARS TO CORRECT ALTIMETRY
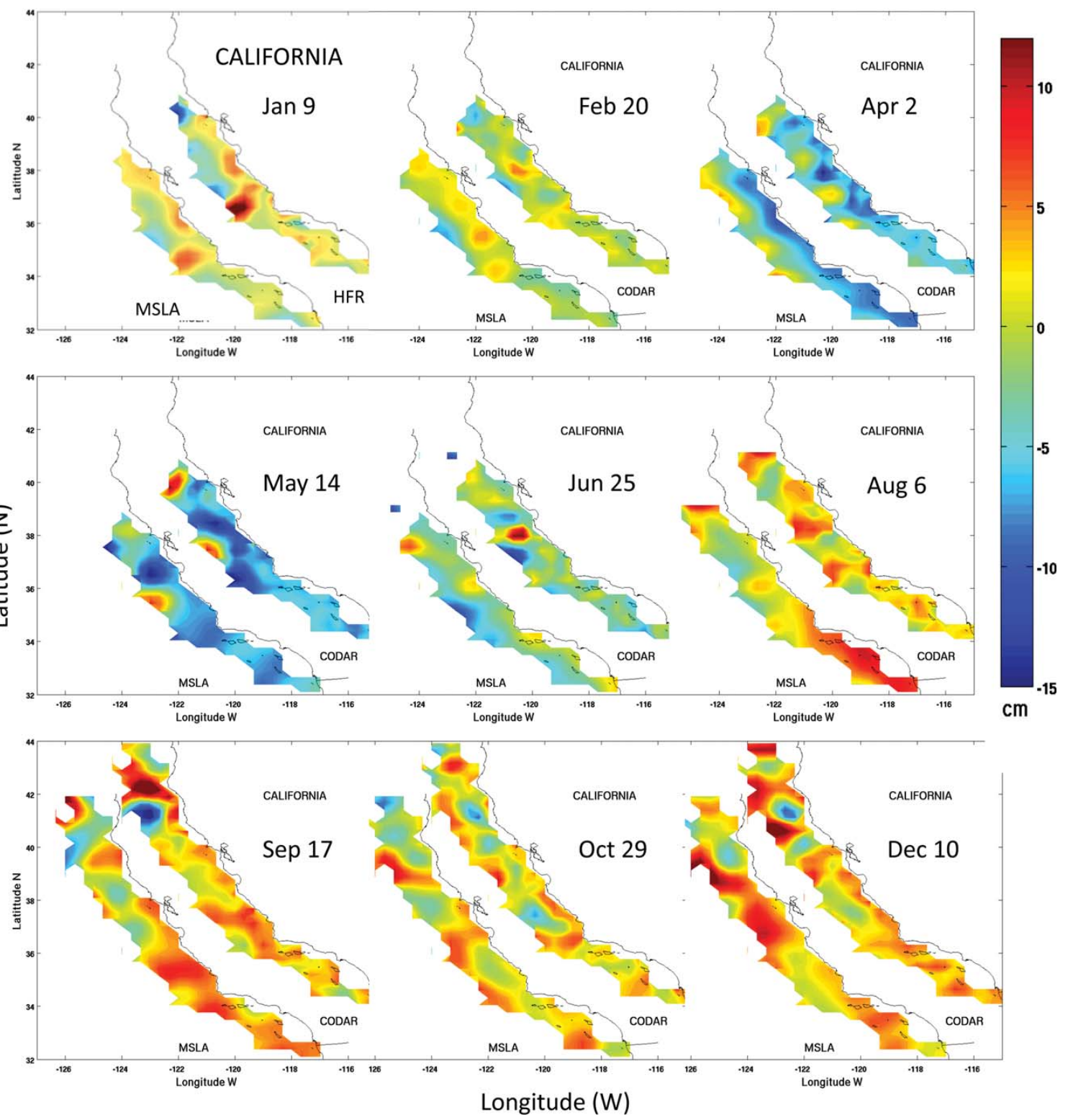

Figure 11. (bottom) Weekly AVISO MSLA compared to (top) HFR SLA every 6 weeks for 2008 along the Californian coastline, on a $1 / 3^{\circ} \times 1 / 3^{\circ}$ grid. The date on the figure represents the center of the week. The SLA is given in centimeters. The HFR coverage increases after August.

extends to the north, because the coverage of the $6 \mathrm{~km}$ HFR grid increases. The time evolution of these two fields for the year 2008 shows excellent agreement: the formation and development of eddies is nearly identical in both series. To quantify this relationship, the SLA time series for the 51 weeks, for each grid point, in the subregion available for the whole year (as observed for the week of 9 January 2008), have been retrieved for both HFR and altimetry. The correlations for the 150 grid points between the two SLA time series (Figure 12) are excellent, except for a few grid points (25 points, $16 \%$ ) where the correlations are lower than 0.7 .
These points are found in the border regions, where the HFR velocities may be less reliable, as well as in the regions less sampled by the HFRs over 2008.

[61] This result, although on a large timescale and lowresolution spatial grid, suggests that the HFR-synthetic SSHs can be used as a proxy for the altimetric-measured heights in the open ocean where the waveforms are, usually, not distorted due to proximity to land.

4.1.2. HFR and Jason-1 SLA

[62] The $6 \mathrm{~km}$ HFR synthetic heights were computed during 2008 and interpolated along the altimeter track P221, which terminates in Monterey Bay, California 


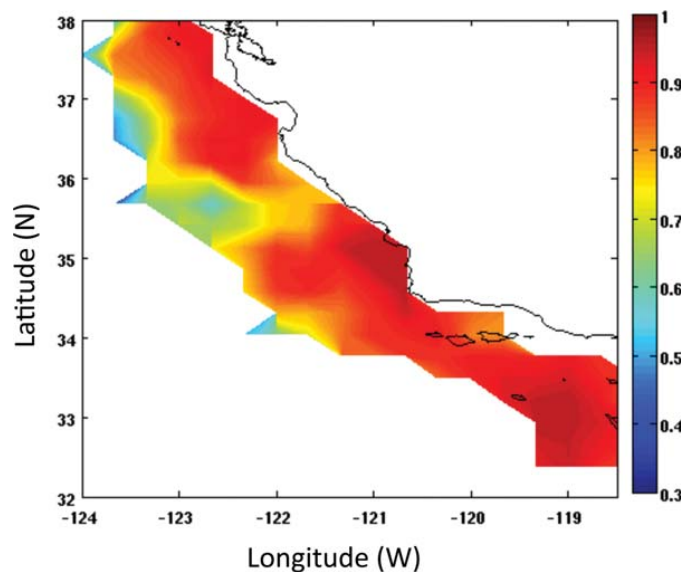

Figure 12. Mean correlation between the time series of the weekly inferred HFR and the MSLA sea levels, for each 150 grid point, on a $1 / 3^{\circ} \times 1 / 3^{\circ}$ grid, over the year 2008 .

(Figure 9). These were compared (first 12 cycles in Figure 13) with the coincident along-track Jason-1 standard openocean $1 \mathrm{~Hz}(6 \mathrm{~km}$ along track spacing) SLA product. The
SLA data are referenced to the same nominal ground track. To reduce the noise from the Jason-1 $1 \mathrm{~Hz}$ set, two different filters have been applied, one with a cutoff wavelength of $50 \mathrm{~km}$ and the other with a cutoff wavelength of $25 \mathrm{~km}$. This procedure enables us to check which level of filtering better correlates with the variability of the SLA signal retrieved from the HFR data set. The means for each time series over the 33 cycles for the year 2008 have been removed (Some Jason-1 data are missing in August).

[63] From this small sample, we can see that in about $70 \%$ of the cases both the HFR sea levels and the altimetric heights agree relatively well (first 12 cycles in Figure 13). The higher wavenumber "wiggles" in the $25 \mathrm{~km}$ filtered curve could depict areas where the SWH is large and the altimeter SLA is retrieved with less precision, or there are simply more detailed dynamical features in this area (for example, Figure 13, C229). In other cases, the two sets diverge in segments that seem time dependent but not related to the distance from the coast (such as Figure 13, C224 and C230). When a $25 \mathrm{~km}$ low-pass filter is applied to the $1 \mathrm{~Hz}$ altimeter SLA the correlation with the $6 \mathrm{~km}$ HFR sea level is low, as the former contains more noise or shorter scales ocean dynamics, and a $50 \mathrm{~km}$ cutoff frequency seems to smooth the data a little too much. In fact, a $40 \mathrm{~km}$ cutoff frequency gives only slightly different

\section{J1 P221 C220 Jan-04}

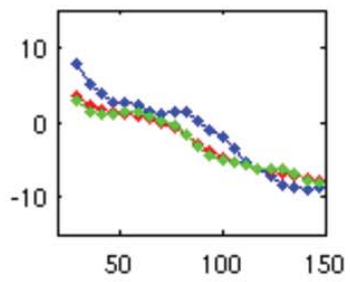

C224 Feb-13

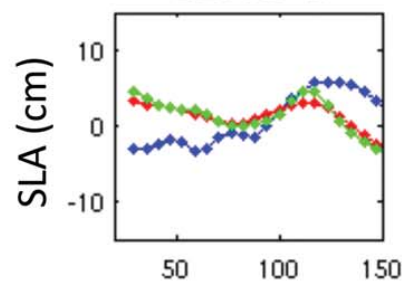

C228 Mar-23

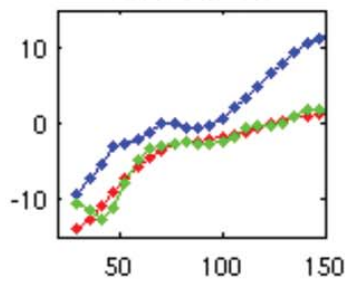

C221 Jan-14

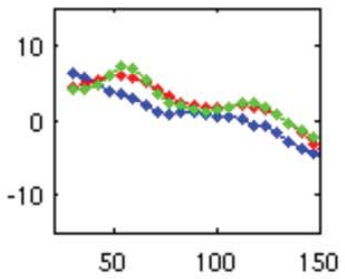

C225 Feb-22

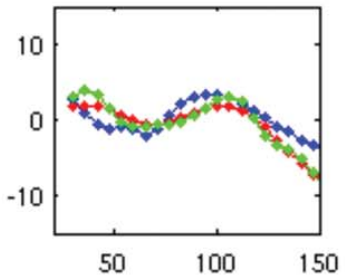

C229 Apr-02

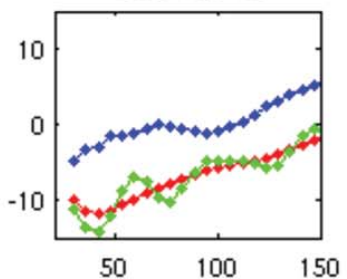

C222 Jan-24
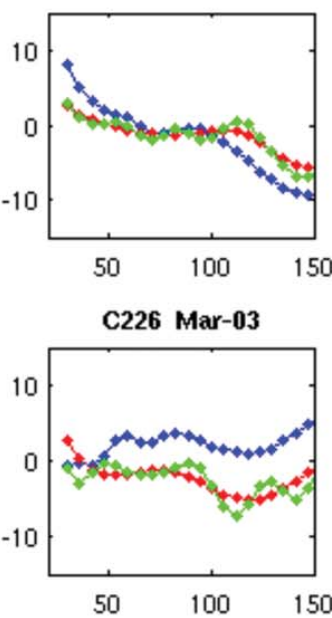

C230 Apr-12

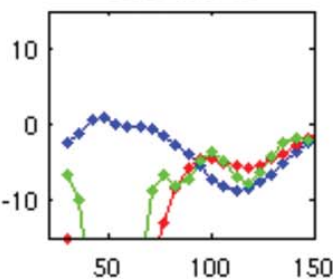

C223 Feb-03
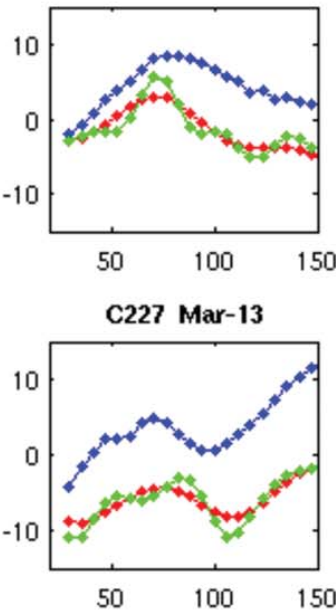

C231 Apr-22

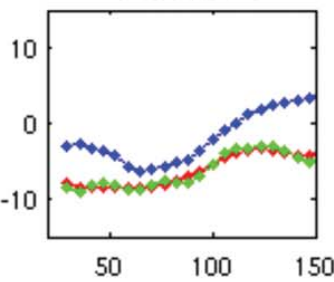

Along track distance to coast $(\mathrm{km})$

Figure 13. Comparison Jason-1 and HFR SLAs along P221 for Cycles 220 to 231. HFR SLAs (in blue) are amplified by 2 and Jason-1 $1 \mathrm{~Hz}$ SLAs are filtered with a cutoff frequency of $50 \mathrm{~km}$ in red and $25 \mathrm{~km}$ in green. 


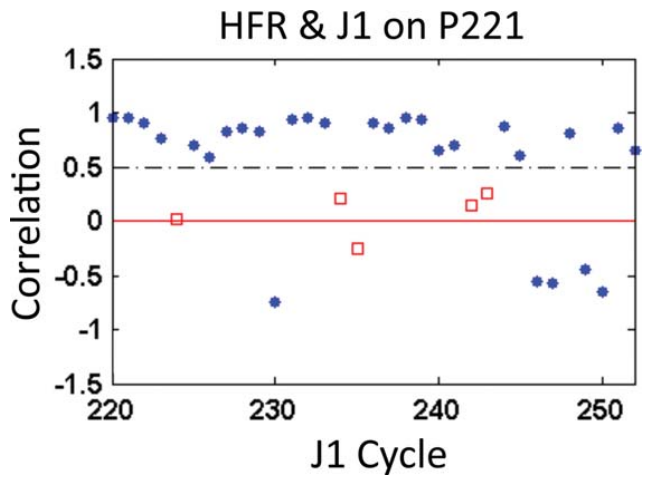

Figure 14. Correlations between the $6 \mathrm{~km}$ HFR inferred SLAs and the Jason-1 SLAs smoothed with a cutoff frequency of $50 \mathrm{~km}$. They are computed along-track P221 on the section $50-150 \mathrm{~km}$ from coast, for 33 cycles in 2008 . Red squares are not statistically significant.

correlations than the $50 \mathrm{~km}$ cutoff frequency. To quantify the relationship the correlation coefficients between the HFR heights and the $50 \mathrm{~km}$ filtered Jason- 1 anomalies have been computed.

[64] The correlation coefficients are calculated (Figure 14) for the 33 cycles during the year 2008 and confirm our conclusions. Five sets are statistically insignificant, five sets are negatively correlated, and the remaining 23 sets have correlations larger than 0.5 . The mean correlation for these 23 sets was 0.82 , with the mean slope of the regression coefficient (HFR versus AVISO) around 2. This explains the consistent multiplication of the HFR-inferred SLA by a factor 2 in order to enhance the comparison.

[65] A closer inspection reveals that for cycle C230, with a correlation of -0.7 , there is a problem with the altimeter data probably from a low-wind event. For cycle C226, a major portion of the sea level variations is in good agreement, but the correlation is only 0.6 because the nearshore end segment diverges. The next step is to find out if this level of matching is sufficient to detect when another retracker would be better suited.

\subsection{Comparison of HFR With PISTACH Retrackers}

[66] In this section, the sea levels from four retrackers are extracted from the PISTACH product. The goal is to determine if we can validate the different retrackers using the sea levels computed from the HFR currents. This comparison will be carried out with two different processing methods for the $20 \mathrm{~Hz}$ altimeter data: one by deriving the traditional $1 \mathrm{~Hz}$ data stream and the other by keeping the $20 \mathrm{~Hz}$ rate. The former will be compared with the sea levels derived from the $6 \mathrm{~km}$ HFR currents only; the latter also includes the $2 \mathrm{~km}$ HFR currents.

\subsubsection{The $1 \mathrm{~Hz}$ PISTACH Data Rate}

[67] Each of the four PISTACH retrackers (MLE-4, Red3, Ice3, and Oce3) SLA is averaged using a 20 point boxcar window and sampled every 20 points, to create 1 $\mathrm{Hz}$ retracked SLAs. In this process, a simple 3 sigma filter, within the 20-point box, edits the extreme outliers. No other special editing following criteria in the GDR handbooks are done, because the goal is to evaluate the perform- ance of the retrackers under various ocean conditions. Then, each retracked SLA series is filtered with a cutoff frequency of 25 and $50 \mathrm{~km}$, and will be analyzed with the coincident $6 \mathrm{~km}$ HFR sea levels. For each cycle and each retracker, an unknown offset has been estimated and removed from the HFR sea levels. This offset has been calculated such that the mean of the differences between the retracked and HFR sea levels, on the track segment considered, is zero.

[68] If we assume the HFR sea levels to be the best estimate of the geostrophic field then we can evaluate the retracking techniques. At this point of study, the shapes of the SSH curves are more important than the exact values, because there may be some offset between HFR and altimetry not yet taken into account. The demeaned SLAs given by the different retrackers are displayed for six consecutive cycles C006-C0011 (Figure 15). The "x," on the figure, points to the retracker that most closely approximates the HFR set. The ovals represent segments where both curves are similar. To quantify the value of having several retrackers at our disposal, the standard deviation (STD) and the correlation coefficient time series between each retracker with the coincident HFR sea level anomalies have been computed for the 49 Jason-2 cycles from C004 to C054 (except for $\mathrm{C} 005$ and $\mathrm{C} 018$ ). The $1 \mathrm{~Hz}$ time series have 17 points on track P221 along a segment from 25 to $120 \mathrm{~km}$ to the coast (corresponding to the along-track distance 50 $150 \mathrm{~km}$, considering the geometry of the Monterey Bay coastline). Table 1 displays the individual results for the six cycles, which represents a comprehensive array of possible encountered situations.

[69] We note that Red3 and MLE-4 are very similar except for cycle $\mathrm{C} 010$. For cycle $\mathrm{C} 008$, all retrackers relate to HFR. Ice 3 has the highest correlation $(\rho)$ of 0.96 . For cycle C006, the shapes differ and Ice 3 performs better, but with a correlation of only 0.4 . For cycle C007, only Ice3 follows the HFR profile. For cycle C009, the end segment fits with MLE-4 or Red3 and have correlations of $\sim 0.66$, but the nearshore segment diverges for all retrackers. For cycle $\mathrm{C} 010$, the $90-150 \mathrm{~km}$ segment is better retracked with Ice 3 or Red3, and closer to shore MLE-4 improves the match, considering the HFR as the validation set. All of the individual retrackers have a low correlation. Finally for cycle C011, all retracked SLA are similar but do not follow HFR data. For these six cycles, the STD for the best-correlated retracker is usually the lowest and is, in these cases, lower than $2 \mathrm{~cm}$ for correlations higher than 0.67 .

[70] The Oce3 is expected to give noise-reduced and improved SLA results. However, the outputs given in the PISTACH product are derived from an early version of the Oce3 algorithm, which contains a slight problem (P. Thibaut, personal communication, Oct. 2012). In this version, the along-track waveform series is divided into contiguous segments. For each segment, an SVD is performed and the same SVD filtering parameters are used for all the waveforms within the segment, before the MLE-3 is applied. But this methodology, as was later discovered, can create retrieved-range jumps between the segments and produce noisy-wavy like Oce3 sea levels (Oce3 C007 in Figure 15). This problem does not affect the quality of Oce 3 for all cycles. When the noise level in the Oce3 (as seen in the 25 $\mathrm{km}$ filtered SLA) is low, the results may be trusted. For 


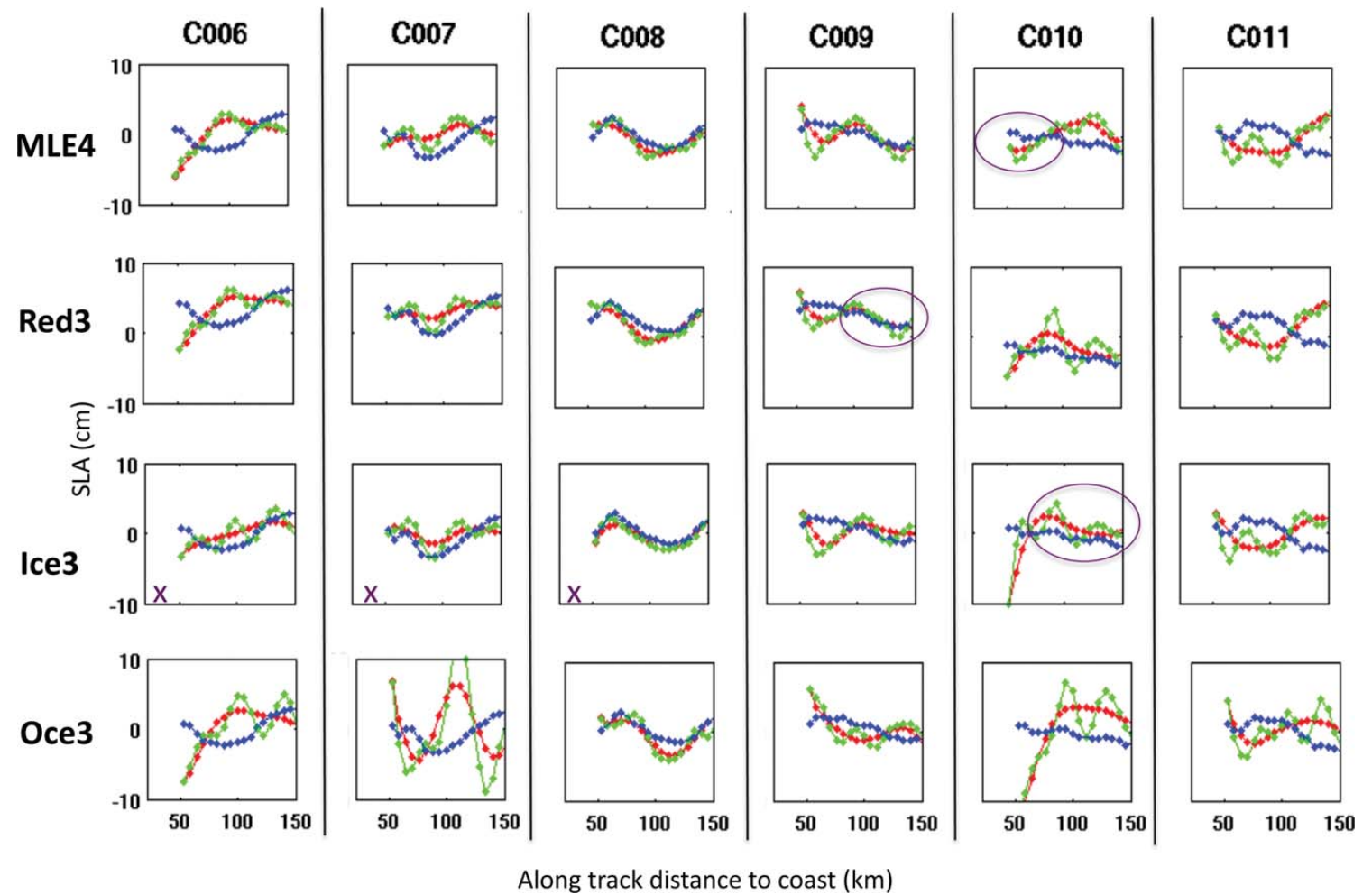

Figure 15. Comparing PISTACH retrackers with HFR sea levels, for six cycles along P221. The $1 \mathrm{~Hz}$ SLAs are smoothed with a cutoff frequency of $50 \mathrm{~km}$ in red and $25 \mathrm{~km}$ in green. The blue curves represent the HFR SLAs amplified by 2 . The $\mathrm{x}$ is the retracker that best fit HFR. The ovals represent segments where both sea level shapes are similar.

example, Oce3 is consistent with the other retrackers for C008 (Figure 15). The results from Oce 3 are considered in this analysis, but with precaution, knowing that its reliability is in question.

[71] In summary and clearly displayed in Figure 16, for MLE-4 and Ice 3 only, there are examples when both match and either fit (Figure 16c) or do not fit (Figure 16d) HFR. There are cases when either MLE-4 (Figure 16a) or Ice3 (Figure 16b) are consistent with HFR. Finally, there are some sets where we could combine segments of MLE-4 and Ice 3 in order to get closer to the, presumed more accu-

Table 1. Correlation Coefficients Between the HFR and PISTACH Retrackers SLAs for Six Cycles in 2008, as Well as the STD of Their Differences

\begin{tabular}{lcccccc}
\hline & C006 & C007 & C008 & C009 & C010 & C011 \\
\hline Correlation & & & & & & \\
MLE-4 & -0.08 & 0.24 & 0.84 & 0.65 & -0.4 & -0.9 \\
Red3 & 0 & 0.66 & 0.82 & $\mathbf{0 . 6 7}$ & 0.03 & -0.87 \\
Ice3 & $\mathbf{0 . 4}$ & $\mathbf{0 . 7 7}$ & $\mathbf{0 . 9 6}$ & 0.07 & -0.44 & -0.82 \\
Oce3 & -0.08 & -0.32 & 0.91 & 0.5 & -0.6 & -0.08 \\
STD (cm) & & & & & & \\
MLE-4 & 3.18 & 2.25 & 1. & 2.1 & 2.34 & 4.25 \\
Red3 & 2.9 & $\mathbf{1 . 8 3}$ & 1.25 & $\mathbf{1 . 6 5}$ & 2.36 & 4.37 \\
Ice3 & $\mathbf{2 . 2 7}$ & 1.85 & $\mathbf{0 . 8 8}$ & 2.3 & 3.11 & 3.75 \\
Oce3 & 4.2 & 6.7 & 1.83 & 2.4 & 5.42 & 3 \\
\hline
\end{tabular}

rate, HFR SSH estimates (Figure 16e). This can be generalized to more retrackers.

[72] Next, the statistics for the 49 cycles are presented in Table 2. The mean correlation for each retracker is around 0.5 . If instead, we create a retracked SLA series, where only the retracker with the highest correlation is kept, the Best-Retracker (B-RTK) sea levels, then the mean of the correlation becomes 0.68 , an amelioration of about $30 \%$. A subset of these 49 cycles is picked by keeping only the BRTK sea level time series with a correlation larger than 0.7. There are $35(70 \%)$ such B-RTK sets. However, for each of the four individual retrackers, the number of sets with a correlation larger than 0.7 is $\sim 25(50 \%) ; 10$ less sets than in the combined B-RTK. The Mean of the correlation for these 35 B-RTK sets is 0.88 compared to 0.77 for the 35 corresponding MLE-4 sets, an improvement of $14 \%$. The B-RTK contains 15 MLE-4, 11 Ice3, 4 Red3, and 5 Oce3 sets with a correlation larger than 0.7 . The mean STD for these sets is $2 \pm 1 \mathrm{~cm}$.

[73] The regular Brown model based retrackers and Ice 3 can give very similar SLA. However, Ice 3 provides better results in several instances. The ice retracker, a threshold type retracker, is not based on a "physically sound" model (not derived from knowledge of microwave scattering at nadir) and care should be taken in its interpretation. This empirical model enables the retracking of waveform shapes that does not conform to the generic open ocean ones and 
ROESLER ET AL.: COASTAL RADARS TO CORRECT ALTIMETRY
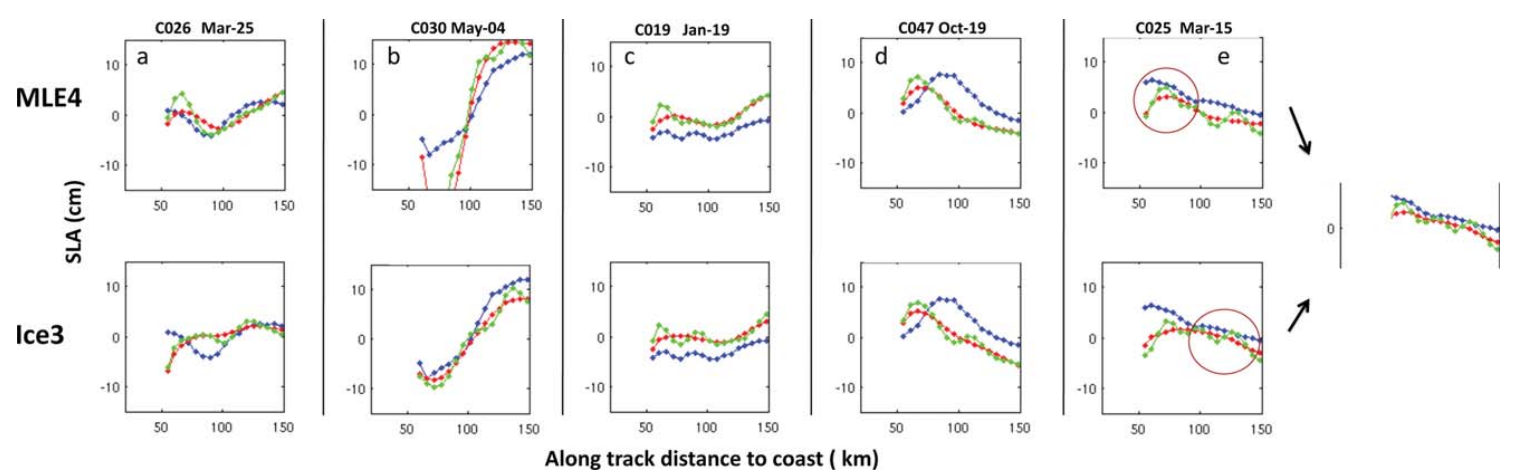

Figure 16. Comparing MLE-4 and Ice 3 with HFR sea levels along P221. Same labeling as in Figure 15. (a) MLE-4 best fit; (b) Ice3 best fit; (c) All similar; (d) MLE-4 and Ice3 similar, but not to HFR; (e) Combining MLE-4 and Ice3 fits HFR.

may give better results under a wider variety of conditions. But the retrieved parameters may not have a clear connection to the underlying geophysical forces. This conclusion underlies the fact that even offshore the waveforms may diverge from the Brown model and the conventional deep ocean retrackers do not apply.

[74] If we assume that the HFR anomalies can be used as a validation tool, these statistics are in favor of the need to have various retrackers at our disposal, even over the continental shelf region. The HFR can provide the backup necessary to determine what are the associated sea surface phenomena that create the disturbances to the waveforms and contribute to less reliable estimates of the conventional open ocean retrackers.

\subsubsection{The $20 \mathrm{~Hz}$ PISTACH Data Rate}

[75] In this section, the PISTACH $20 \mathrm{~Hz}$ data stream will be studied employing a different processing to start examining the possibility of extracting a higher-resolution product nearshore, where the temporal and spatial variability of ocean processes increases. Instead of subsampling to the 1 $\mathrm{Hz}$ data rate, the original full $20 \mathrm{~Hz}$ rate is used. These data are noisy. To reduce the measurement noise, the $20 \mathrm{~Hz}$ SLA outliers are removed by using an iterative strategy that combines a low-pass filter with a 3 sigma boundary editing. Then the data is smoothed using a boxcar window of 21 points $(7 \mathrm{~km})$ and 60 points $(21 \mathrm{~km})$. Filtering the data with a cutoff frequency of $7 \mathrm{~km}$ will, still, create a noisy along-track sea-level series. It was kept partly to reveal regions with more high-frequency errors that may be associated with a variable sea state or other disturbing conditions. Now we present SLA data sets that go all the way to the shore, as the level of filtering is more appropriate to deal with the end points. We can compare them with the 2 $\mathrm{km}$ derived HFR SSH available for the year 2009 along P221. In fact, now the nearshore $2 \mathrm{~km}$ HFR currents and offshore $6 \mathrm{~km}$ ones are combined. These observations are used to generate the $2 \mathrm{~km}$ OI gridded HFR sea levels that are then interpolated every $2 \mathrm{~km}$ ( $\sim 6$ points) on the alongtrack P221.

[76] The $2 \mathrm{~km}$ HFR sea levels have more variability than the previous $6 \mathrm{~km}$ product (Figure 19). The question is do these reflect the structures observed in altimetry. The 21$\mathrm{km}$ filtered PISTACH sea levels contain small high-frequency components that seem unrealistic (Figure 19). Their larger spatial-scale dynamics agree well with the ones of HFR, especially for the case C031 on May 14, for Ice3. For C034 on June 12, the nearshore segment $0-60 \mathrm{~km}$ contains small-scale features in the HFR sea levels that correspond to the variations of Ice3. If those features are realistic, then filtering altimeter data at this level can be beneficial in the nearshore regions.

\subsection{Sea State}

[77] Various sea state conditions can affect the quality of altimetric SSH. This can explain some of the disagreements between the HFR sea levels and those from altimetry. In this discussion, Oce3 is not considered. It is not easy to

Table 2. Correlation Coefficients and STD of the Differences Between the HFR and PISTACH Retrackers SLAs for All 49 Cycles (Top) and for a Subset of 35 Cycles Chosen Such That the Correlation for the B-RTK Is Larger Than 0.7 (Bottom) ${ }^{\mathrm{a}}$

\begin{tabular}{|c|c|c|c|c|c|}
\hline & MLE-4 & Red3 & Ice3 & Oce3 & $\begin{array}{l}\text { B-RTK (Retracker With } \\
\text { the Highest } \rho \text { ) }\end{array}$ \\
\hline \multicolumn{6}{|c|}{ For All 49 Cycles } \\
\hline Correlation & $0.48 \pm 0.51$ & $0.5 \pm 0.47$ & $0.47 \pm 0.47$ & $0.42 \pm 0.51$ & $0.68 \pm 0.34$ \\
\hline $\mathrm{STD}(\mathrm{cm})$ & $3 \pm 1.9$ & $2.9 \pm 1.5$ & $2.6 \pm 1.1$ & $3.6 \pm 2$ & $2 \pm 1$ \\
\hline \multicolumn{6}{|c|}{ For 35 Cycles When $\rho$ of $B-R T K>0.7$} \\
\hline Correlation & $0.77 \pm 0.2$ & $0.77 \pm 0.17$ & $0.68 \pm 0.36$ & $0.67 \pm 0.32$ & $0.88 \pm 0.09$ \\
\hline STD $(\mathrm{cm})$ & $2.7 \pm 1.9$ & $2.7 \pm 1.7$ & $2.45 \pm 1.2$ & $3.2 \pm 2$ & $2 \pm 1$ \\
\hline
\end{tabular}

${ }^{\mathrm{a}}$ Each value is the mean \pm STD 
a)

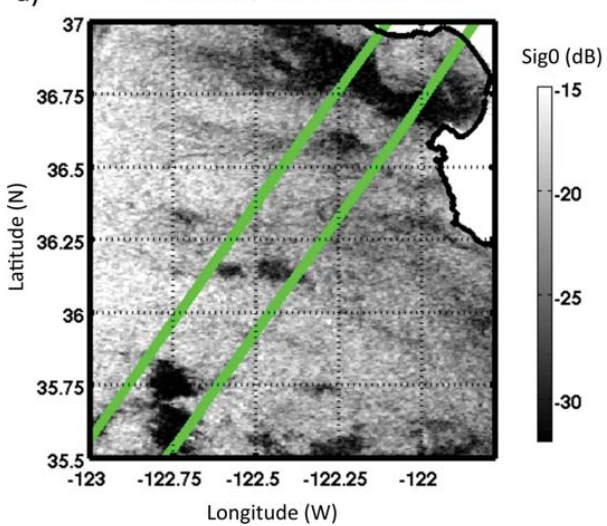

b)

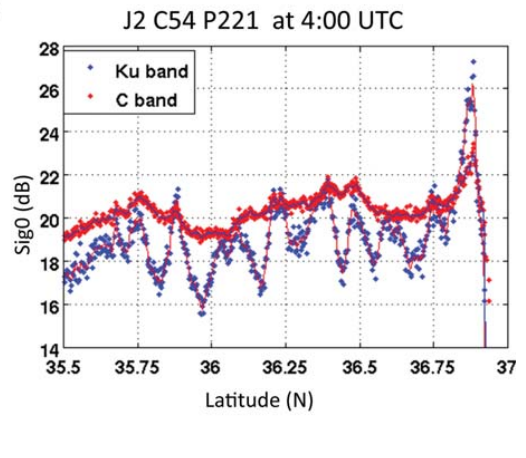

Figure 17. Backscattering coefficient sig0 for 28 December 2009 off Monterey Bay, derived from (a) Envisat ASAR at 06:00 UTC (b) Jason-2 for the Ku-band (blue), and C-band (red) at 04:00 UTC. The lines on the SAR image approximate the extent of Jason-2 P221 circular footprint.

generalize, but cases of high SWH or high Sig0 can disturb the outputs of the retrackers. For instance, the presence of unusually high-Sig0 values $(\operatorname{Sig} 0>16 \mathrm{~dB}$ for Jason-2 MLE-4) in the altimeter footprint from Sig0-bloom events may signal a breakdown in the typical Brown model. The bloom events, along P221 in Monterey Bay, extend over a few tens to hundreds of kilometers. Their occurrence and frequency vary from cycle to cycle. There have been about $20 \%$ of large bloom events during the Jason- 2 time series considered. As can be seen in the ENVISAT SAR image on 28 December 2009 (Figure 17) $2 \mathrm{~h}$ apart from the Jason2 C054 P221 passage, small-scale variations in surface roughness over the altimeter footprint can occur. The altimeter MLE-4 retrieved Sig0 for the Ku-band and C-band are very high $(>16 \mathrm{~dB})$ with wavy patterns, related to the dark patches of low-SAR backscatter. Under low wind conditions $(\leq 5 \mathrm{~m} / \mathrm{s})$ short gravity waves can be suppressed and a high-altimeter specular backscatter coincides with a low Bragg scattering mechanism in SAR. The knowledge of a high-resolution repartition of surface roughness over the altimeter footprint is important. During a bloom event, Ice3 behaves in a more stable manner than MLE-4 (or Red3) and stays closer to the HFR sea levels.

[78] Cycle C030, on May 4 (Figure 18), is a case when the Sig0-bloom event extends over a large region with Sig0 $>20 \mathrm{~dB}$; none of the filtered $20 \mathrm{~Hz}$ retrackers are well adapted in this situation. The HFR sea levels, available throughout, have large variations $(-15$ to $+15 \mathrm{~cm})$. Can they be used to correct altimetry during this bloom event? We mentioned, in section 2.2, that there can be a lack of HFR data in case of low wind events, but these have been observed to last less than a few hours over 2009. By doing a 3 day average, we can still get an estimate of the SSHs. In this case, for $\mathrm{C} 030$, the answer is probably yes, because, interestingly, at the $1 \mathrm{~Hz}$ data rate Ice 3 corrected this bloom event very well (Figure 16).

[79] Note that we chose to use the MLE-4 derived Sig0, as an indication of the sea state or problematic zone, from a well-studied, traditional open ocean retracker. Historically, MLE-4 was implemented to correct for the Jason-1 attitude problem, and estimates the slope of the trailing edge related to the mispointing angle. It was then chosen to retrack Jason-2 echoes. MLE-4 gives better estimates of range and SWH, relative to MLE-3, but Sig0 is degraded because the joint estimation of the mispointing and Sig0 is ill conditioned [Thibaut et al., 2010]. As a reminder, the new GDR version " $D$ " includes both the MLE-3 and MLE-4 outputs and their Sig0 have different characteristics. For example,

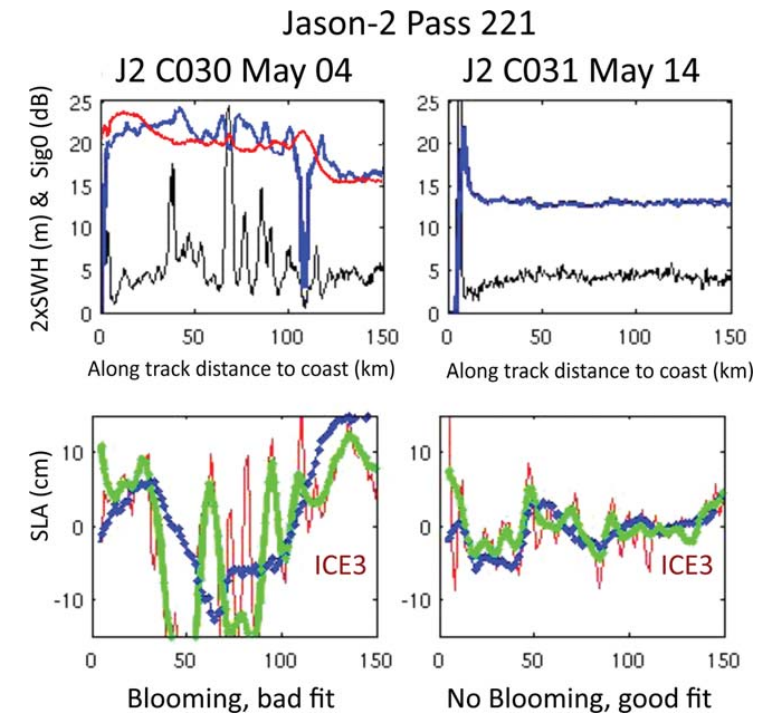

Figure 18. Comparison HFR and Ice3 SLAs along P221, (left) with and (right) without bloom. (top) Sig0 (dB) from MLE-4 in blue, SWH (m) amplified by 2 in black. Bloom events occur for Sig0 $>16 \mathrm{~dB}$ for Jason-2. Close to shore there is contamination by land. For $\operatorname{Sig} 0 \sim 0 \mathrm{~dB}$, there is a loss of signal by the tracker. Sig0 from Red 3 is displayed in red for C030. (bottom) HFR SLAs are amplified by 2 , in blue; Jason-2 $20-\mathrm{Hz}$ Ice3 SLAs are filtered with a cutoff frequency of $7 \mathrm{~km}$ in red and $21 \mathrm{~km}$ in green. 
J2 C026 Mar 25
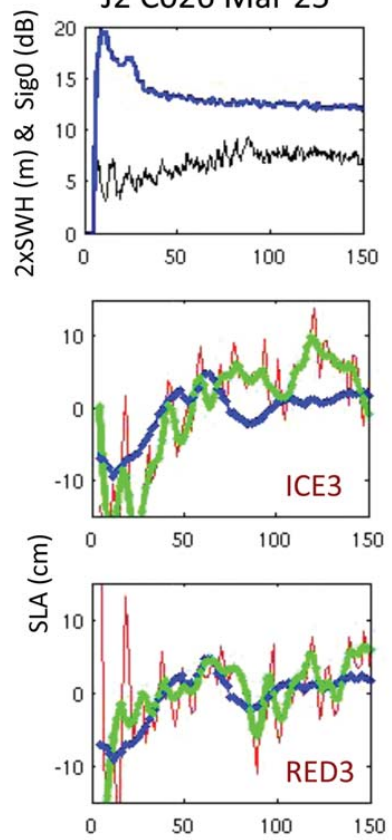

Jason-2 Pass 221 J2 C031 May 14
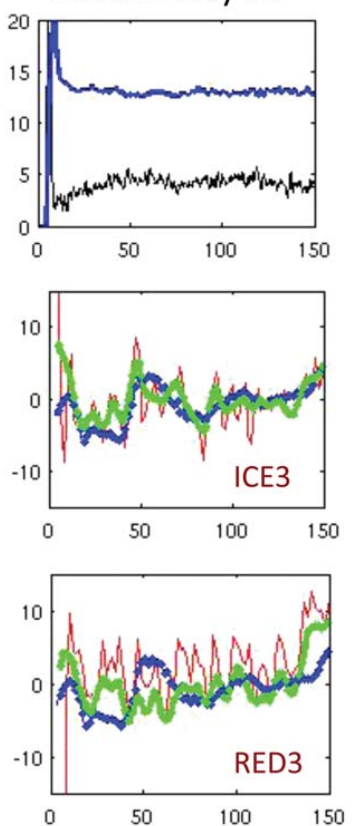

Along track distance to coast $(\mathrm{km})$
J2 C034 Jun 12
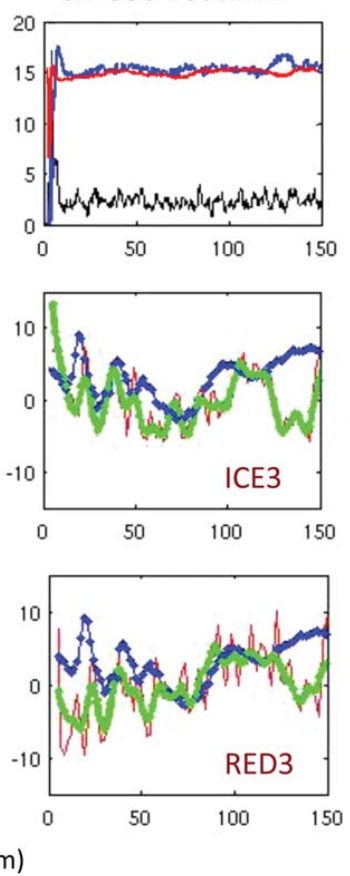

Figure 19. Comparing (middle) Jason-2 ICE3 and (bottom) RED3 retrackers with HFR SLAs for different sea states along P221 (same color code as in Figure 18). Sig0 from Red3 is displayed for C034 in red.

Figure 18 shows the Sig0 profiles for MLE-4 and Red3 (which is based on MLE-3) during a bloom event. The MLE-4 derived Sig0 contains more noise level and one can observe more undulations. The Red 3 and MLE-4 derived SWH exhibit very similar behavior in the cases presented, so only the MLE-4 SWH is displayed.

[80] We examined three cases, along P221, to compare the response of the Ice 3 and Red 3 retrackers depending on the sea state as described by the altimeter MLE-4 derived SWH and Sig0 (Figure 19):

[81] (1) Cycle C031: There are no bloom events, Sig0 stays below $15 \mathrm{~dB}$, and $\mathrm{SWH}$ below $2.5 \mathrm{~m}$. Ice 3 performs well and better than Red3 throughout.

[82] (2) Cycle C026: Beyond the close to shore bloom events $(<30 \mathrm{~km})$ Red3 performs better though it seems noisy. In this case, the SWH is relatively high, starting at $2.5 \mathrm{~m}$ and increasing to $5 \mathrm{~m}$ offshore.

[83] (3) Cycle C034: Low SHW $<1.5 \mathrm{~m}$. Ice 3 matches well closer than $50 \mathrm{~km}$, then Red3 until a little bloom event around $140 \mathrm{~km}$. For this example, the Red3 Sig0 is displayed, because it has an opposite behavior in the region of the bloom event as seen by MLE-4. Any difference may signal a breakdown of the assumed Brown model waveforms.

[84] To summarize, using only Ice 3 and MLE-4, for Sig0-bloom events, Ice3 is more stable. When both SWH is low and Sig0 is less than $16 \mathrm{~dB}$, the sea levels from MLE-4 and Ice 3 are very similar (Figure 10, C019). When the
$\mathrm{SWH}$ is high $(\mathrm{SWH}>3 \mathrm{~m}$, associated with low Sig0, high wind speeds) or SWH is variable within the segment, Ice3 and MLE-4 can differ. It these instances MLE-4 seems to better fit the HRF sea levels (Figure 10, C016). Although we generalized, along a track the best fit relative to HFR sea levels can change in an unpredictable fashion, it is not clear under which conditions one retracker would be more efficient than the other. This needs to be investigated more systematically to see if we could predict a trend.

[85] As mentioned in the last section, there are cases when only one segment of a retracker fits the HFR sea levels and cases when the HFR and altimeter sea level sets diverge. Besides the bloom events, there are about 15 cases when both data sets are not quite similar, either as a phase shift or as an end segment. One of the future tasks will be to determine which one is a better representation of the true sea surface level. There are limitations inherent to the HFR measurements and OI processing that need to be better evaluated to define how effectively we could use the HFR sea levels as a reference to improve the quality of altimetry.

\section{Discussion and Conclusion}

[86] One of the challenges of using satellite altimetry in the coastal ocean is correcting for distortions of the altimetric waveforms linked to the presence of possible rapid changes in sea states and/or the presence of land within the 
altimeter footprint. Many retracking procedures have been developed, but there is great difficulty in knowing what is the proper method and where it is best applied. We evaluated the skills of the HFR coastal surface currents to validate the retrackers, especially in the region $25-150 \mathrm{~km}$ offshore. The U.S. West Coast HFR network monitors hourly ocean surface currents with an offshore range up to $150 \mathrm{~km}$ and spatial resolutions of 2 and $6 \mathrm{~km}$ depending on the radar operating frequency. By analyzing the time- and space scales of the coastal oceanic features, we can fit a stream function to the HFR coastal currents to retrieve their matching SSHs, which are mapped with varying spatial scales using OI. Tested on regions more than $25 \mathrm{~km}$ off the California coast, we demonstrate a similarity between the HF coastal radar derived SSH fields and those computed directly from standard satellite altimetry product using Jason-1 and Jason-2 over the years 2008 and 2009.

[87] The behavior of four retrackers from Jason-2 PISTACH coastal product were analyzed and showed the possibility of determining which retracker better fits the HFR sea levels depending on the sea state and other conditions. The PISTACH version of Oce 3 is not reliable. Red3 and MLE-4 give very similar results, for the sea level, over the offshore regions. This is not surprising as both retrackers are based on the MLE fits using the Brown model. Red3 uses observations only centered on the leading edge instead of the complete waveform. Red3 will provide better estimates of the sea level when the trail end part is distorted. The largest differences are seen between MLE-4 and Ice3. Ice3 is more stable when the waveforms depart from the standard open ocean shape, for example, during Sig0bloom events. But Ice3 is not based on a physical model, so care must be taken in its interpretation.

[88] Having the HFR sea levels to validate the retrackers demonstrated a tendency to have Ice3 fit better in cases of bloom events and MLE-4 (or Red3) in cases of high SWH. There are cycles when the match is almost perfect. There are also instances when the HFR and altimetry do not agree, either on segments or for the complete track considered. If we keep a time series of best fitted retracker (B-RTK), relative to HFR, then the mean correlation, at the $1 \mathrm{~Hz}$ level, is higher than 0.7 , seventy percent of the time along-track P221 on the portion $25 \mathrm{~km}$ to $120 \mathrm{~km}$ offshore. The mean correlation for this $70 \%$ subset is 0.88 . This result is without combining several retrackers on an individual track.

[89] A different processing strategy was implemented on the altimeter data, to use the original full $20 \mathrm{~Hz}$ rate, instead of subsampling to $1 \mathrm{~Hz}$. The $20 \mathrm{~Hz}$ SLA outliers are edited with an iterative strategy that combines a lowpass filter with a 3 sigma boundary editing. Then the data is smoothed with a $21 \mathrm{~km}$ boxcar window. At this level of filtering, the SLAs are still contaminated by high-frequency signals. But we find that it contains additional information about oceanic processes, in the coastal zone, by referencing them to the $2 \mathrm{~km}$ resolution HFR sea levels.

[90] The processing of both data sets still requires improvements to make them more compatible and understand the limitations of this comparison. For instance there are some issues with the HFR surface currents inversion methodology. Three-day-averaged HFR currents are used as input to the OI, but perhaps a 1 day average would be

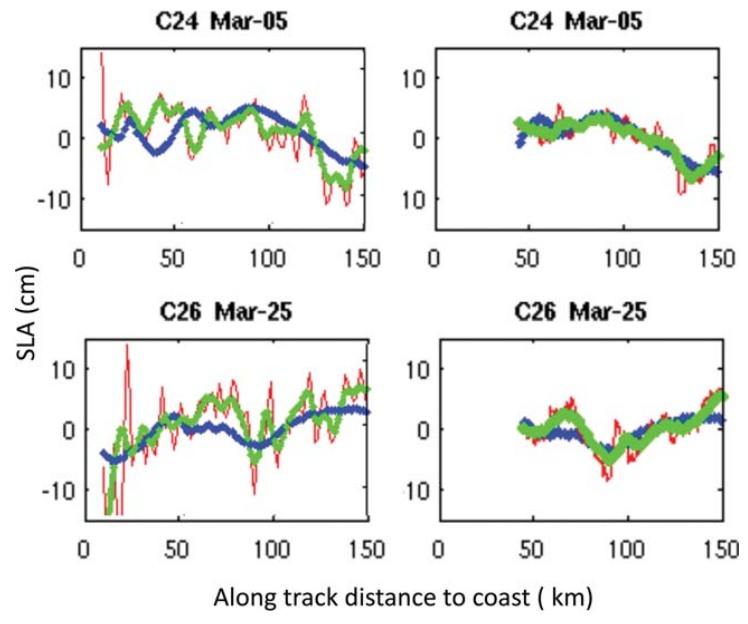

Figure 20. (left) No editing and (right) edited MLE-4 with HFR sea levels (in blue). The MLE-4 sea levels are both filtered with a cutoff frequency of $7 \mathrm{~km}$ in red and 21 $\mathrm{km}$ in green. But on the right, spurious data are removed, before filtering, and refines the $20 \mathrm{~Hz}$ MLE-4 SLAs.

more representative of the instantaneous altimeter alongtrack SSH. How stringent should the quality of the HFR currents be, which would lead to data gaps in the HR coverage? Finally there is the issue of a variable HFR synthetic heights multiplicative factor around 2, in order to bring them to the same signal level as those from altimetry. What is its origin, and how should it be accounted for in the inversion process? Perhaps, a comparison between HFR currents and altimetric geostrophic currents can help us resolve this problem.

[91] For the altimeter processing, the concerns are about editing (depending on the goals) and finding the best filtering to match the HFR heights variations. For example, spurious data, known to be less reliable offshore $(\mathrm{Sig} 0>16 \mathrm{~dB}$ and/or SWH $>5 \mathrm{~m}$ ), are edited from the $20 \mathrm{~Hz}$ MLE-4 data before filtering with a $21 \mathrm{~km}$ boxcar window. This results in a noise-reduced altimeter SLA (Figure 20). This strategy can be used to compare HFR sea levels with altimetry in situations when, only, reliable altimeter measurements are required.

[92] There are limitations inherent to the HFR data that need to be better evaluated to define how effectively it can detect the erroneous altimetric data and be used as a reference to improve their quality. Some dissimilarity, between the HFR/altimetric SSH comparisons, arises because the principal measurements of both instruments capture different physical phenomena. To address the last point, a reliable and quantifiable correspondence between the altimetric SSH and the independent inferred SSH should be established. This can be done first in the offshore regions, in situations when the standard-retracked altimeter data are reliable. This means that, in a first step, the altimeter data will give feedback on the validity of the technique, as well as how to improve the HFR sea levels. In a second step, the cases when the two sets diverge will be analyzed and checked to determine which one is a better estimate of the true sea surface parameter; by looking at the waveforms, or 


\section{ROESLER ET AL.: COASTAL RADARS TO CORRECT ALTIMETRY}

by introducing auxiliary information (sea surface temperature, ocean color). The influence of the seasonal currents or wind patterns can also be examined on the quality of the HFR sea levels. Then the analysis can be extended toward the coastline.

[93] Overall, these preliminary studies have demonstrated the value of HFR surface currents as a promising tool to validate and find new strategies to correct coastal altimetric SSHs. They also provide some insight into the altimeter behavior as a function of ocean conditions to account for some oceanic mechanisms and various sea states, particular to the continental shelf, that affect the conventional altimeter measurements. The optimal method for altimetry retracking seems to be a combination of different retrackers for various segments of the altimeter track and depends on the cycle. The experience gained from a systematic comparison of both data sets can provide hints on how to correct conventional coastal altimetry when no HFR arrays are deployed. Maybe we can find a relationship between the behavior of the waveform series around the point of concern and the quality of the fit HFR with altimetry $\mathrm{SSH}$; or possibly a relationship between the Ku-band derived Sig0 and the C-band derived Sig0 can help detect when the conventional ocean retracker is not optimal and refine the choice of retracker. These clues will apply to the immediate oceanic area.

[94] The implementation of the methodology to other coastal regions will depend on the specifics of the regional coastal zone dynamics. The parameters between HFR and altimetry SSH may need some adjustments, not only in the spatial scales used in the OI but also in the linear correspondence between the two sets. For instance the continental shelf in the North Atlantic Bight, along the U.S. east coast, is very wide and shallow relative to the one on the U.S. West Coast. This study is a guide on what should be done in other areas.

[95] There are cases when the match between altimetric and HFR along-track sea levels is almost perfect and cannot be coincidence only. Once the conditions for a consistency between the HFR and altimeter data are established, the HFR surface currents would be invaluable for the validation of future altimetry missions, better suited for coastal regions, when those conditions are met.

[96] Acknowledgments. This work is supported by NASA's Ocean Science Topography Team with Eric Lindstrom as the program manager. Sung Yong Kim is supported by the Human Resources Development of the Korea Institute of Energy Technology Evaluation and Planning (KETEP), Ministry of Trade, Industry and Energy (No. 20114030200040), Republic of Korea. We would like to acknowledge valuable discussion with Jean Touranadre of IFREMER relating to Sig0-blooms. We also thank three anonymous reviewers for their constructive comments and suggestions. The altimetry MSLA data were produced by SSALTO DUACS, PISTACH and DT SLA data by CLS, distributed by AVISO with support from CNES. High-frequency radar-derived surface current data originate from the following universities and research organizations in California: Scripps Institution of Oceanography at University of California, San Diego, University of Southern California, Marine Science Institute at University of California, Santa Barbara, California Polytechnic State University, Naval Postgraduate School, Romberg Tiburon Center at San Francisco State University, Humboldt State University, and Bodega Marine Laboratory at University of California, Davis.

\section{References}

Andersen, O. B., and R. Scharroo (2011), Range and Geophysical corrections in coastal regions and implications for mean sea surface determi- nation, in Coastal Altimetry, edited by S. Vignudelli et al., pp.103-145, Springer, Berlin, doi:10.1007/978-3-642-12796-0

AVISO/Altimetry (2010), Coastal and Hydrology Altimetry Product (PISTACH) Handbook, CLS-DOS-NT-10-246, SALP-MU-P-OP-16031-CN 01/00, ed.1.0, CNES/CLS, France, http://www.aviso.oceanobs.com/file admin/documents/data/tools/hdbk_Pistach.pdf.

AVISO/Altimetry (2012), SSALTO/DUACS Users Handbook DT CorSSH and DT SLA Product, CLS-DOS-NT-086.381, ed. 2.0. CNES/CLS, France, http://www.aviso.oceanobs.com/fileadmin/documents/data/ tools/hdbk dt corssh dt sla.pdf.

AVISO/Altimetry (2013), SSALTO/DUACS Users Handbook MSLA and MADT Near-Real Time and Delayed Time Products, CLS-DOS-NT06.034, SALP-MU-P-EA-21065-CLS, ed.3.4, CNES/CLS, France, http://www.aviso.oceanobs.com/fileadmin/documents/data/tools/hdbk duacs.pdf.

Barrick, D. E., M. W. Evans, and B. L. Weber (1977), Ocean surface currents mapped by radar, Science, 198, 138-144, doi:10.1126 science.198.4313.138

Birol, F., M. Cancet, and C. Estournel (2010), Aspects of the seasonal variability of the northern current (NW Mediterranean Sea) observed by altimetry, J. Mar. Syst., 81, pp. 297-311, doi:10.1016/ j.jmarsys.2010.01.005.

Bouffard, J., S. Vignudelli, M. Hermann, F. Lyard, Marsaleix, Y. Ménard, and P. Cipollini (2008), Comparison of ocean dynamics with a regional circulation model and improved altimetry in the North-western Mediterranean, Terr. Atmos. Ocean. Sci., 19, 117-133, doi:10.3319/ TA0.2008.19.1-2117(SA)

Bretherton, F. D., R. E. Davis, and C. B. Fandry (1976), A technique for objective analysis and design of oceanographic experiments applied to MODE-73, Deep Sea Res., 23, 559-582.

Brown, G. (1977), The average impulse response of a rough surface and its applications, IEEE Trans. Antennas Propag., 25, 67-74.

Brown, S. (2010), A novel near-land radiometer wet path-delay retrieval algorithm: Application to the Jason-2/OSTM advanced microwave radiometer, IEEE Trans. Geosci. Remote Sens., 48(4), 1986-1992, doi:10.1109/TGRS.2009.2037220.

Chavanne, C. P., and P. Klein (2010), Can oceanic submesoscale processes be observed with satellite altimetry?, Geophys. Res. Lett., 37, L22602, doi:10.1029/2010GL045057.

Chelton, D., J. Ries, B. Haines, L. Fu, and P. Callahan (2001), Satellite altimetry, in Satellite Altimetry and Earth Sciences: A Handbook of Techniques and Applications, edited by L. Fu and A. Cazenave, pp. 1-132, Elsevier, New York.

Chereskin, T. K., and M. Trunnell (1996), Correlation scales, objective mapping, and absolute geostrophic flow in the California Current, $J$. Geophys. Res., 101, 22,619-22,629, doi:10.1029/96JC02004.

Cipollini, P., S. Vignudelli, F. Lyard, and L. Roblou (2008), 15 years of altimetry at various scales over the Mediterranean, in Remote Sensing of European Seas, edited by V. Barale and M. Gade, pp. 296-306, Springer, Heidelberg, Germany, doi:10.1007/978-1-4020-6772-3-22.

Davis, C. H. (1997), A robust threshold retracking algorithm for measuring ice-sheet surface elevation change from satellite radar altimetry, IEEE Trans. Geosci. Remote Sens., 35(4), 974-979.

Deng, X. (2004), Improvement of geodetic parameter estimation in coastal regions from satellite radar altimetry, Ph.D. thesis, 248 pp., Curtin Univ. of Technol., Perth, Australia.

Deng, X., and W. E. Featherstone (2006), A coastal retracking system for satellite radar altimeter waveforms: Application to ERS-2 around Australia, J. Geophys. Res., 111, C06012, doi:10.1029/2005JC003039.

Deng, X., W. E. Featherstone, C. Hwang, and P. A. M. Berry (2002), Estimation of contaminations of ERS-2 and POSEIDON satellite radar altimetry close to the coasts of Australia, Mar. Geod., 25(4), 249-271.

Deng, X., W. E. Featherstone, C. Hwang, and C. Shum (2003), Improved coastal marine gravity anomalies at the Taiwan Strait from altimeter waveform retracking, in International Association of Geodesy Symposia, vol. 126, edited by C. Hwang, C. Shum, and J. Li, pp. 41-45, Springer, Berlin, Heidelberg.

Desporte, C., E. Obligis, and L. Eymard (2007), On the wet tropospheric correction for altimetry in coastal regions, IEEE Trans. Geosci. Remote Sens., 45(7), 2139-2149, doi:10.1109/TGRS.2006.888967.

Gómez-Enri, J., I. Caballero, G. Navarro, and P. Villares (2011), Towards a validation of ENVISAT RA-2 high rate significant wave height in coastal systems: Case study of the Gulf of Cadiz, paper presented at 5th EARSeL Workshop on Remote Sensing of the Coastal Zone, Prague, Czech Republic, June 2011. 


\section{ROESLER ET AL.: COASTAL RADARS TO CORRECT ALTIMETRY}

Gommenginger, C., P. Thibaut, L. Fenoglio-Marc, X. Deng, J. GómezEnri, and Y. Gao (2011), Retracking altimeter waveforms near the coasts, in Coastal Altimetry, edited by S. Vignudelli et al., pp.61-10, Berlin, Springer, doi:10.1007/978-3-642-12796-0.

Herbert, G., N. Ayoub, P. Marsaleix, and F. Lyard (2011), Signature of the coastal circulation variability in altimetric data in the Southern Bay of Biscay during winter and fall 2004, J. Mar. Sys., v88, 2, 139-158, doi:10.1016/j.jmarsys.2011.03.004.

Hickey, B. M. (1998), Coastal oceanography of Western North America from the tip of Baja California to Vancouver Island, The Sea, 11, 345-393.

Hwang, C., and S. A. Chen (2000), Circulation eddies over the South China Sea derived from Topex/Poseidon altimetry, J. Geophys. Res., 105 , $23,943-23,965$

Hwang, C., J. Guo, X. Deng, H. Y. Hsu, and Y. Liu (2006), Coastal gravity anomaly from retracked Geosat/GM altimetry: Improvement, limitation and role of airborne gravity data, J. Geod., 80, 204-216, doi:10.1007/ s00190-006-0052-x.

Ikeda, M., W. J. Emery, and L. A. Mysak (1984), Seasonal variability in meanders of the California current system off Vancouver Island, J. Geophys. Res., 89, 3487-3505, doi:10.1029/JC089iC03p03487.

Kim, S. Y. (2010), Observations of submesoscale eddies using high-frequency radar-derived kinematic and dynamic quantities, Cont. Shelf Res., 30, 1639-1655, doi:10.1016/j.csr.2010.06.011.

Kim, S. Y., E. J. Terrill, and B. D. Cornuelle (2008), Mapping surface currents from HF radar radial velocity measurements using optimal interpolation, J. Geophys. Res., 113, C10023, doi:10.1029/2007JC004244.

Kim, S. Y., et al. (2011), Mapping the U.S. West Coast surface circulation: A multiyear analysis of high-frequency radar observations, J. Geophys. Res., 116, C03011, doi:10.1029/2010JC006669

Laws, K. E., and J. D. Paduan (2011), Error assessment of HF radar based ocean current measurements: An error model based on sub-period measurement variance, paper presented at IEEE/OES Current, Waves and Turbulence Measurement Conference, Monterey, Calif.

Lebedev, N. A., A. G. Kostianoy, A. I. Ginzburg, D. P. Medvenev, N. A Sheremet, and S. N. Shauro (2011), Satellite altimetry applications in the Barents and White Sea, in Coastal Altimetry, edited by S. Vignudelli et al., pp. 389-415, Springer, Berlin doi:10.1007/978-3-642-12796-0.

Lee, H., C. Shum, Y. Yi, M. Ibaraki, J. Kim, A. Braun, C. Kuo, and Z. Lu (2009), Louisiana wetland and water level monitoring using retracked TOPEX/POSEIDON altimetry, Mar. Geod., 32, 284-284, doi:10.1080/ 0149041090394767.

Lee H., C. Shum, W. Emery, X. Deng, C. Roesler, Y. Yi, C. Kuo, Z. Lu, and S. Calmant (2010), Validation of Jason-2 altimeter data by waveform retracking over California coastal ocean, Mar. Geod., 33(1), 304 316, doi: $10.1080 / 01490419.2010 .488982$

Le Hénaff, M., L. Roblou, and J. Bouffard (2010), Characterizing the Navidad current interannual variability using coastal altimetry, Ocean Dyn., 61, 425-437, doi: 10.1007/s10236-010-0360-9.

Lipa, B. J., and D. E. Barrick (1986), Extraction of sea state from HF radar sea echo: Mathematical theory and modeling, Radio Sci., 21(1), 81-100, doi:10.1029/RS021i001p00081.

Liu Y., R.H. Weisberg, S. Vignudelli, L. Roblou and C.R.Merz (2012), Comparison of the X-TRACK altimetry estimated currents with moored ADCP and HF radar observations on the West Florida Shelf, Adv. Space Res., 50(8), 1085-1098, doi:10.1016/j.asr.2011.09.012.

Lyard, F., F. Lefèvre, T. Letellier, and O. Francis (2006), Modelling the global ocean tides: Modern insights from FES2004, Ocean Dyn., $56(5-$ 6), 394-415, doi:10.10007/s10236-006-0086-x.
Mitchum, G., D. Hancock, G. Hayne, and D. Vandemark (2004), Blooms of Sig0 in the Topex radar altimeter data, J. Atmos. Oceanic Technol., 21,1232-1245, doi:10.1175/1520-04226(2004)021.

Morrow, R., and P. Y. Le Traon (2006), 15 years of satellite altimetry and Mesoscale Ocean Dynamics observed with 15 years of altimetric data, paper presented at the Symposium on 15 Years of Progress in Radar Altimetry, Venice, Italy, ESA.

Ohlmann C., P. White, L. Washburn, B. Emery, E. Terrill, and M. Otero (2007), Interpretation of coastal HF radar-derived surface currents with high-resolution drifter data, J. Atmos. Oceanic Technol., 24, 666-680, doi: 10.1175/JTECH1998.1.

OSTM/Jason-2 Products Handbook (2011), SALP_MU M OP_15815_CN, ed1.8, CNES, France, http://www.aviso.oceanobs. com fileadmin/documents/data/tools/hdbk_j2.pdf.

Paduan, J. D., and H. C. Graber (1997), Introduction to high-frequency radar: Reality and myth, Oceanography, 10, 36-39.

Paduan, J. D., K. C. Kim, M. S. Coock, and F. P. Chavez (2006), Calibration and validation of direction-finding high-frequency radar ocean surface current observations, IEEE J. Oceanic Eng., 31(4), 862-875, doi: 10.1109/JOE.2006.886195.

Quartly, G. D., M. A. Srokosz, and T. H. Guymer (1998), Understanding the effects of rain on radar altimeter waveforms, Adv. Space Res., 22(11), 1567-1570.

Ruiz S., A. Pascual, B. Garau, I. Pujol, and J. Tintoré (2009), Vertical motion in the upper ocean from glider and altimetry data, Geophys. Res. Lett., 26, L14607, doi:10.1029/2009GL038569.

Saraceno M., P. T. Strub, and P. M. Korso (2008), Estimates of sea surface height and near-surface alongshore coastal currents from combinations of altimeters and tide gauges, J. Geophys. Res., 113, C11013, doi: 10.1029/2008JC004756.

Severini, J. (2010), Estimation et classification de Signaux Altimétriques, Ph.D. thesis, p.160, IPN, Toulouse, France.

Strub, P. T., Kosro, P. M., and A. Huyer (1991), The nature of the cold filaments in the California Current System, J. Geophys. Res., 96, 14,74314,768 .

Thibaut, P., J. C. Poisson, E. Bronner, and N. Picot (2010), Relative performance of the MLE3 and MLE4 retracking algorithms on Jason-2 altimeter waveforms, Mar. Geod., 33, 1, 317-335, doi:10.1080/ 01490419.2010 .491033

Tisch, T. D., S. R. Ramp, and C. A. Collins (1992), Observations of the geostrophic current and water mass characteristics off Point Sur, California, from May 1988 through November 1989, J. Geophys. Res., 92 12,535-12,555, doi:10.1029/92JC01094.

Tournadre, J., B. Chapron, N. Reul, and D. C. Vandemark (2006), A satellite altimeter model for ocean slick detection, J. Geophys. Res., 111 , C04004, doi:10.1029/2005JC03109.

Tran, N., S. Labroue, S. Phillips, E. Bronner, and N. Picot (2010), Overview and update of the sea state bias corrections for the Jason-2, Jason-1 and TOPEX missions, Mar. Geod., 33(S1), 348-362, doi:10.1080/ 01490419.2010.487788

Walstadt, L. J., J. S. Allen, P. M. Korso, and A. Huyer (1991), Dynamics of the coastal transition zone through data assimilation studies, $J$ Geophys Res, 96, 14,956-14,977.

Wilkin, J. L., M. S. Bowen, and W. J. Emery (2002), Mapping mesoscale currents by optimal interpolation of satellite radiometer and altimeter data, Ocean Dyn., 52(3), 95-103, doi:10.1007/s10236-001-0011-2. 